\title{
Relay-Selection Improves the Security-Reliability Trade-Off in Cognitive Radio Systems
}

Yulong Zou, Senior Member, IEEE, Benoit Champagne, Senior Member, IEEE, Wei-Ping Zhu, Senior Member, IEEE, and Lajos Hanzo, Fellow, IEEE

5 Abstract - We consider a cognitive radio (CR) network consisting 6 of a secondary transmitter (ST), a secondary destination (SD) and 7 multiple secondary relays (SRs) in the presence of an eavesdropper, 8 where the ST transmits to the SD with the assistance of SRs, while 9 the eavesdropper attempts to intercept the secondary transmission. 10 We rely on careful relay selection for protecting the ST-SD trans11 mission against the eavesdropper with the aid of both single-relay 12 and multi-relay selection. To be specific, only the "best" SR is cho13 sen in the single-relay selection for assisting the secondary trans14 mission, whereas the multi-relay selection invokes multiple SRs for 15 simultaneously forwarding the ST's transmission to the SD. We 16 analyze both the intercept probability and outage probability of 17 the proposed single-relay and multi-relay selection schemes for the 18 secondary transmission relying on realistic spectrum sensing. We 19 also evaluate the performance of classic direct transmission and 20 artificial noise based methods for the purpose of comparison with 21 the proposed relay selection schemes. It is shown that as the inter22 cept probability requirement is relaxed, the outage performance of 23 the direct transmission, the artificial noise based and the relay se-

24 lection schemes improves, and vice versa. This implies a trade-off 25 between the security and reliability of the secondary transmission 26 in the presence of eavesdropping attacks, which is referred to as 27 the security-reliability trade-off (SRT). Furthermore, we demon28 strate that the SRTs of the single-relay and multi-relay selection 29 schemes are generally better than that of classic direct trans30 mission, explicitly demonstrating the advantage of the proposed 31 relay selection in terms of protecting the secondary transmissions 32 against eavesdropping attacks. Moreover, as the number of SRs 33 increases, the SRTs of the proposed single-relay and multi-relay

Manuscript received May 7, 2014; revised August 21, 2014 and October 16, 2014; accepted November 27, 2014. This work was partially supported by the Priority Academic Program Development of Jiangsu Higher Education Institutions, the National Natural Science Foundation of China (Grant Nos. 61302104 and 61401223), the Scientific Research Foundation of Nanjing University of Posts and Telecommunications (Grant Nos. NY213014 and NY214001), the 1311 Talent Program of Nanjing University of Posts and Telecommunications, the Natural Science Foundation of Jiangsu Province (Grant No. BK20140887), and the Programme de bourses d'excellence pour etudiants etrangers (PBEEE) of the Government of Quebec. The associate editor coordinating the review of this paper and approving it for publication was $\mathrm{H}$. $\mathrm{Li}$

Y. Zou is with the School of Telecommunications and Information Engineering, Nanjing University of Posts and Telecommunications, Nanjing 210003, China (e-mail: yulong.zou@njupt.edu.cn).

B. Champagne is with the Department of Electrical \& Computer Engineering, McGill University, Montreal, QC H3A 1Y1, Canada (e-mail: benoit. champagne@mcgill.ca).

W.-P. Zhu is with the Department of Electrical \& Computer Engineering, Concordia University, Montreal, QC H3G 1M8, Canada (e-mail: weiping@ece. concordia.ca).

L. Hanzo is with the Department of Electronics and Computer Science, University of Southampton, Southampton SO17 1BJ, U.K. (e-mail: 1h@ecs. soton.ac.uk).

Color versions of one or more of the figures in this paper are available online at http://ieeexplore.ieee.org.

Digital Object Identifier 10.1109/TCOMM.2014.2377239 selection approaches significantly improve. Finally, our numerical 34 results show that as expected, the multi-relay selection scheme 35 achieves a better SRT performance than the single-relay selection. 36

Index Terms-Security-reliability trade-off, relay selection, 37 intercept probability, outage probability, eavesdropping attack, 38 cognitive radio.

\section{INTRODUCTION}

$\mathbf{T}$ HE security aspects of cognitive radio (CR) systems [1]- 41 [3] have attracted increasing attention from the research 42 community. Indeed, due to the highly dynamic nature of the CR 43 network architecture, legitimate CR devices become exposed 44 to both internal as well as to external attackers and hence they 45 are extremely vulnerable to malicious behavior. For example, 46 an illegitimate user may intentionally impose interference (i.e. 47 jamming) for the sake of artificially contaminating the CR envi- 48 ronment [4]. Hence, the CR users fail to accurately characterize 49 their surrounding radio environment and may become misled 50 or compromised, which leads to a malfunction. Alternatively, 51 an illegitimate user may attempt to tap the communications of 52 authorized CR users by eavesdropping, to intercept confidential 53 information.

Clearly, CR networks face diverse security threats during 55 both spectrum sensing [5], [6] as well as spectrum sharing [7], 56 spectrum mobility [8] and spectrum management [9]. Extensive 57 studies have been carried out for protecting CR networks both 58 against primary user emulation (PUE) [10] and against denial- 59 of-service (DoS) attacks [11]. In addition to PUE and DoS at- 60 tacks, eavesdropping is another main concern in protecting the 61 data confidentiality [12], although it has received less attention 62 in the literature on CR network security. Traditionally, crypto- 63 graphic techniques are employed for guaranteeing transmission 64 confidentiality against an eavesdropping attack. However, this 65 introduces a significant computational overhead [13] as well as 66 imposing additional system complexity in terms of the secret 67 key management [14]. Furthermore, the existing cryptographic 68 approaches are not perfectly secure and can still be decrypted 69 by an eavesdropper (E), provided that it has the capacity to carry 70 out exhaustive key search with the aid of brute-force attack [15]. 71

Physical-layer security [16], [17] is emerging as an efficient 72 approach for defending authorized users against eavesdropping 73 attacks by exploiting the physical characteristics of wireless 74 channels. In [17], Leung-Yan-Cheong and Hellman demon- 75 strated that perfectly secure and reliable transmission can be 76 achieved, when the wiretap channel spanning from the source 77 to the eavesdropper is a further degraded version of the main 78 
79 channel between the source and destination. They also showed 80 that the maximal secrecy rate achieved at the legitimate desti81 nation, which is termed the secrecy capacity, is the difference 82 between the capacity of the main channel and that of the 83 wiretap channel. In [18]-[20], the secrecy capacity limits of 84 wireless fading channels were further developed and character85 ized from an information-theoretic perspective, demonstrating 86 the detrimental impact of wireless fading on the physical87 layer security. To combat the fading effects, both multiple-input 88 multiple-output (MIMO) schemes [21], [22] as well as coop89 erative relaying [23]-[25] and beamforming techniques [26], 90 [27] were investigated for the sake of enhancing the achievable 91 wireless secrecy capacity. Although extensive research efforts 92 were devoted to improving the security of traditional wireless 93 networks [16]-[27], less attention has been dedicated to CR 94 networks. In [28] and [29], the achievable secrecy rate of 95 the secondary transmission was investigated under a specific 96 quality-of-service (QoS) constraint imposed on the primary 97 transmission. Additionally, an overview of the physical-layer 98 security aspects of CR networks was provided in [30], where 99 several security attacks as well as the related countermeasures 100 are discussed. In contrast to conventional non-cognitive wire101 less networks, the physical-layer security of CR networks has to 102 consider diverse additional challenges, including the protection 103 of the primary user's QoS and the mitigation of the mutual 104 interference between the primary and secondary transmissions. 105 Motivated by the above considerations, we explore the 106 physical-layer security of a CR network comprised of a sec107 ondary transmitter (ST) communicating with a secondary des108 tination (SD) with the aid of multiple secondary relays (SRs) 109 in the presence of an unauthorized attacker. Our main focus 110 is on investigating the security-reliability trade-off (SRT) of 111 the cognitive relay transmission in the presence of realistic 112 spectrum sensing. The notion of the SRT in wireless physical113 layer security was introduced and examined in [31], where the 114 security and reliability was characterized in terms of the inter115 cept probability and outage probability, respectively. In contrast 116 to the conventional non-cognitive wireless networks studied in 117 [31], the SRT analysis of CR networks presented in this work 118 additionally takes into account the mutual interference between 119 the primary user (PU) and secondary user (SU).

120 The main contributions of this paper are summarized as 121 follows.

- We propose two relay selection schemes, namely both single-relay and multi-relay selection, for protecting the secondary transmissions against eavesdropping attacks. More specifically, in the single-relay selection (SRS) scheme, only a single relay is chosen from the set of multiple SRs for forwarding the secondary transmissions from the ST to the SD. By contrast, the multi-relay selection (MRS) scheme employs multiple SRs for simultaneously assisting the ST-SD transmissions.

- We present the mathematical SRT analysis of the proposed SRS and MRS schemes in the presence of realistic spectrum sensing. Closed-form expressions are derived for the intercept probability (IP) and outage probability (OP) of both schemes for transmission over Rayleigh fading channels. The numerical SRT results of conventional direct

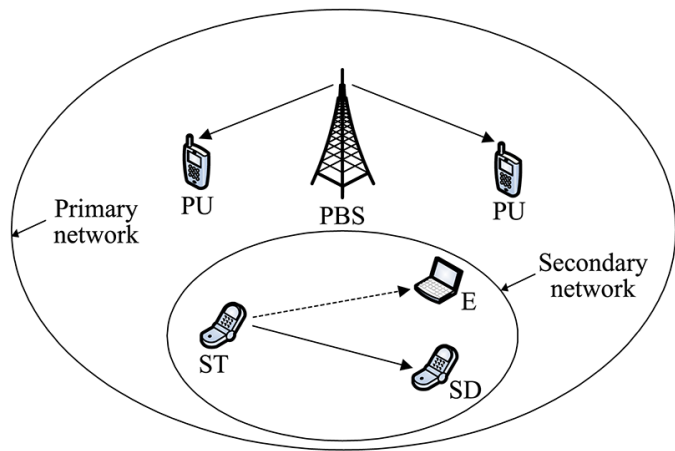

PBS: Primary Base Station PU: Primary User

ST: Secondary Transmitter SD: Secondary Destination

E: Eavesdropper

Fig. 1. A primary wireless network in coexistence with a secondary CR network.

transmission and artificial noise based schemes are also 137 provided for comparison purposes.

- It is shown that as the spectrum sensing reliability is 139 increased and/or the false alarm probability is reduced, the 140 SRTs of both the SRS and MRS schemes are improved. 141 Numerical results demonstrate that the proposed SRS and 142 MRS schemes generally outperform the conventional di- 143 rect transmission and artificial noise based approaches in 144 terms of their SRTs.

The remainder of this paper is organized as follows. 146 Section II presents the system model of physical-layer security 147 in CR networks in the context of both the direct transmission as 148 well as the SRS and MRS schemes. In Section III, we analyze 149 the SRTs of these schemes in the presence of realistic spectrum 150 sensing over Rayleigh fading channels. Next, numerical SRT 151 results of the direct transmission, SRS and MRS schemes are 152 given in Section IV, where the SRT performance of the artificial 153 noise based scheme is also numerically evaluated for com- 154 parison purposes. Finally, Section V provides our concluding 155 remarks.

II. Relay Selection Aided Protection Against 157 EAVESDROPPING IN CR NETWORKS

We first introduce the overall system model of physical-layer 159 security in CR networks. We then present the signal model of 160 the conventional direct transmission approach, which will serve 161 as our benchmarker, as well as of the SRS and MRS schemes 162 for improving the CR system's security against eavesdropping 163 attacks.

\section{A. System Model}

As shown in Fig. 1, we consider a primary network in 166 coexistence with a secondary network (also referred to as a $C R 167$ network). The primary network includes a primary base station 168 (PBS) and multiple primary users (PUs), which communicate 169 with the PBS over the licensed spectrum. By contrast, the 170 secondary network consisting of one or more STs and SDs 171 exploits the licensed spectrum in an opportunistic way. To 172 
173 be specific, a particular ST should first detect with the aid 174 of spectrum sensing whether or not the licensed spectrum is 175 occupied by the PBS. If so, the ST is not at liberty to transmit 176 to avoid interfering with the PUs. If alternatively, the licensed 177 spectrum is deemed to be unoccupied (i.e. a spectrum hole 178 is detected), then the ST may transmit to the SD over the 179 detected spectrum hole. Meanwhile, E attempts to intercept the 180 secondary transmission from the ST to the SD. For notational 181 convenience, let $H_{0}$ and $H_{1}$ represent the event that the licensed 182 spectrum is unoccupied and occupied by the PBS during a 183 particular time slot, respectively. Moreover, let $\hat{H}$ denote the 184 status of the licensed spectrum detected by spectrum sensing. 185 Specifically, $\hat{H}=H_{0}$ represents the case that the licensed 186 spectrum is deemed to be unoccupied, while $\hat{H}=H_{1}$ indicates 187 that the licensed spectrum is deemed to be occupied.

188 The probability $P_{d}$ of correct detection of the presence of $189 \mathrm{PBS}$ and the associated false alarm probability $P_{f}$ are defined 190 as $P_{d}=\operatorname{Pr}\left(\hat{H}=H_{1} \mid H_{1}\right)$ and $P_{f}=\operatorname{Pr}\left(\hat{H}=H_{1} \mid H_{0}\right)$, respectively. 191 Due to the background noise and fading effects, it is impossible 192 to achieve perfectly reliable spectrum sensing without missing 193 the detection of an active PU and without false alarm, which 194 suggests that a spectral band is occupied by a PU, when it 195 is actually unoccupied. Moreover, the missed detection of the 196 presence of PBS will result in interference between the PU 197 and SU. To guarantee that the interference imposed on the 198 PUs is below a tolerable level, both the successful detection 199 probability (SDP) $P_{d}$ and false alarm probability (FAP) $P_{f}$ 200 should be within a meaningful target range. For example, the 201 IEEE 802.22 standard requires $P_{d}>0.9$ and $P_{f}<0.1$ [2]. For 202 better protection of PUs, we consider $P_{d}=0.99$ and $P_{f}=0.01$, 203 unless otherwise stated. Additionally, we consider a Rayleigh 204 fading model for characterizing all the channels between any 205 two nodes of Fig. 1. Finally, all the received signals are assumed 206 to be corrupted by additive white Gaussian noise (AWGN) 207 having a zero mean and a variance of $N_{0}$.

\section{B. Direct Transmission}

209 Let us first consider the conventional direct transmission 210 as a benchmark scheme. Let $x_{p}$ and $x_{s}$ denote the random 211 symbols transmitted by the PBS and the ST at a particular 212 time instance. Without loss of generality, we assume $E\left[\left|x_{p}\right|^{2}\right]=$ $213 E\left[\left|x_{S}\right|^{2}\right]=1$, where $E[\cdot]$ represents the expected value operator. 214 The transmit powers of the PBS and ST are denoted by $P_{p}$ and $215 P_{S}$, respectively. Given that the licensed spectrum is deemed to 216 be unoccupied by the PBS (i.e. $\hat{H}=H_{0}$ ), ST transmits its signal $217 x_{S}$ at a power of $P_{S}$. Then, the signal received at the SD can be 218 written as

$$
y_{d}=h_{s d} \sqrt{P_{s}} x_{s}+h_{p d} \sqrt{\alpha P_{p}} x_{p}+n_{d}
$$

219 where $h_{s d}$ and $h_{p d}$ represent the fading coefficients of the 220 channel spanning from ST to SD and that from PBS to SD, 221 respectively. Furthermore, $n_{d}$ represents the AWGN received at $222 \mathrm{SD}$ and the random variable (RV) $\alpha$ is defined as

$$
\alpha= \begin{cases}0, & H_{0} \\ 1, & H_{1},\end{cases}
$$

where $H_{0}$ represents that the licensed spectrum is unoccupied 223 by PBS and no primary signal is transmitted, leading to $\alpha=0.224$ By contrast, $H_{1}$ represents that PBS is transmitting its signal $x_{p} 225$ over the licensed spectrum, thus $\alpha=1$. Meanwhile, due to the 226 broadcast nature of the wireless medium, the ST's signal will 227 be overheard by E and the overheard signal can be expressed as 228

$$
y_{e}=h_{s e} \sqrt{P_{s}} x_{s}+h_{p e} \sqrt{\alpha P_{p}} x_{p}+n_{e},
$$

where $h_{s e}$ and $h_{p e}$ represent the fading coefficients of the 229 channel spanning from ST to $\mathrm{E}$ and that from PBS to E, 230 respectively, while $n_{e}$ represents the AWGN received at E. 231 Upon combining Shannon's capacity formula [31] with (1), we 232 obtain the capacity of the ST-SD channel as

$$
C_{s d}=\log _{2}\left(1+\frac{\left|h_{s d}\right|^{2} \gamma_{s}}{\alpha\left|h_{p d}\right|^{2} \gamma_{p}+1}\right)
$$

where $\gamma_{s}=P_{s} / N_{0}$ and $\gamma_{p}=P_{p} / N_{0}$. Similarly, the capacity of the 234 ST-E channel is obtained from (3) as

$$
C_{s e}=\log _{2}\left(1+\frac{\left|h_{s e}\right|^{2} \gamma_{s}}{\alpha\left|h_{p e}\right|^{2} \gamma_{p}+1}\right) \text {. }
$$

\section{Single-Relay Selection}

In this subsection, we consider the cognitive relay network 237 of Fig. 2, where both SD and E are assumed to be beyond 238 the coverage area of the ST [24], [25], and $N$ secondary 239 relays (SRs) are employed for assisting the cognitive ST-SD 240 transmission. We assume that a common control channel (CCC) 241 [6] is available for coordinating the actions of the different 242 network nodes and the decode-and-forward (DF) relaying using 243 two adjacent time slots is employed. More specifically, once 244 the licensed spectrum is deemed to be unoccupied, the ST first 245 broadcasts its signal $x_{s}$ to the $N$ SRs, which attempt to decode 246 $x_{s}$ from their received signals. For notational convenience, let 247 $\mathcal{D}$ represent the set of SRs that succeed in decoding $x_{s}$. Given 248 $N$ SRs, there are $2^{N}$ possible subsets $\mathcal{D}$, thus the sample space 249 of $\mathcal{D}$ is formulated as

$$
\Omega=\left\{\emptyset, \mathcal{D}_{1}, \mathcal{D}_{2}, \cdots, \mathcal{D}_{n}, \cdots, \mathcal{D}_{2^{N-1}}\right\}
$$

where $\emptyset$ represents the empty set and $\mathcal{D}_{n}$ represents the $n$-th 251 non-empty subset of the $N$ SRs. If the set $\mathcal{D}$ is empty, implying 252 that no SR decodes $x_{s}$ successfully, then all the SRs remain 253 silent and thus both SD and E are unable to decode $x_{s}$ in this 254 case. If the set $\mathcal{D}$ is non-empty, a specific $\mathrm{SR}$ is chosen from 255 $\mathcal{D}$ to forward its decoded signal $x_{s}$ to SD. Therefore, given 256 $\hat{H}=H_{0}$ (i.e. the licensed spectrum is deemed unoccupied), ST 257 broadcasts its signal $x_{S}$ to $N$ SRs at a power of $P_{S}$ and a rate of 258 $R$. Hence, the signal received at a specific $\mathrm{SR}_{i}$ is given by

$$
y_{i}=h_{s i} \sqrt{P_{s}} x_{s}+h_{p i} \sqrt{\alpha P_{p}} x_{p}+n_{i},
$$

where $h_{s i}$ and $h_{p i}$ represent the fading coefficients of the ST-SR 260 channel and that of the $\mathrm{PBS}_{-} \mathrm{SR}_{i}$ channel, respectively, with 261 
$262 n_{i}$ representing the $\mathrm{AWGN}$ at $\mathrm{SR}_{i}$. From (7), we obtain the 263 capacity of the $\mathrm{ST}_{-} \mathrm{SR}_{i}$ channel as

$$
C_{s i}=\frac{1}{2} \log _{2}\left(1+\frac{\left|h_{s i}\right|^{2} \gamma_{s}}{\alpha\left|h_{p i}\right|^{2} \gamma_{p}+1}\right),
$$

264 where the factor $\frac{1}{2}$ arises from the fact that two orthogonal time 265 slots are required for completing the message transmission from $266 \mathrm{ST}$ to $\mathrm{SD}$ via $\mathrm{SR}_{i}$. According to Shannon's coding theorem, 267 if the data rate is higher than the channel capacity, the re268 ceiver becomes unable to successfully decode the source signal, 269 regardless of the decoding algorithm adopted. Otherwise, the 270 receiver can succeed in decoding the source signal. Thus, using 271 (8), we can describe the event of $\mathcal{D}=\emptyset$ as

$$
C_{s i}<R, \quad i \in\{1,2, \cdots, N\} .
$$

272 Meanwhile, the event of $\mathcal{D}=\mathcal{D}_{n}$ is described as

$$
\begin{aligned}
& C_{s i}>R, \quad i \in \mathcal{D}_{n} \\
& C_{s j}<R, \quad j \in \overline{\mathcal{D}}_{n},
\end{aligned}
$$

273 where $\overline{\mathcal{D}}_{n}$ represents the complementary set of $\mathcal{D}_{n}$. Without 274 loss of generality, we assume that $\mathrm{SR}_{i}$ is chosen within $\mathcal{D}_{n}$ to 275 transmit its decoded result $x_{s}$ at a power of $P_{s}$, thus the signal 276 received at SD can be written as

$$
y_{d}=h_{i d} \sqrt{P_{s}} x_{s}+h_{p d} \sqrt{\alpha P_{p}} x_{p}+n_{d},
$$

277 where $h_{i d}$ represents the fading coefficient of the $\mathrm{SR}_{i}-\mathrm{SD}$ 278 channel. From (11), the capacity of the $\mathrm{SR}_{i}-\mathrm{SD}$ channel is 279 given by

$$
C_{i d}=\frac{1}{2} \log _{2}\left(1+\frac{\left|h_{i d}\right|^{2} \gamma_{s}}{\alpha\left|h_{p d}\right|^{2} \gamma_{p}+1}\right),
$$

280 where $i \in \mathcal{D}_{n}$. In general, the specific $\mathrm{SR}_{i}$ having the highest 281 instantaneous capacity to SD is chosen as the "best" SR for as282 sisting the ST's transmission. Therefore, the best relay selection 283 criterion is expressed from (12) as

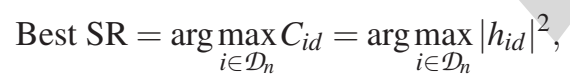

284 which shows that only the channel state information (CSI) $\left|h_{i d}\right|^{2}$ 285 is required for performing the relay selection without the need 286 for the eavesdropper's CSI knowledge. Upon combining (12) 287 and (13), we obtain the capacity of the channel spanning from 288 the "best" SR to SD as

$$
C_{b d}=\frac{1}{2} \log _{2}\left(1+\frac{\gamma_{s}}{\alpha\left|h_{p d}\right|^{2} \gamma_{p}+1} \max _{i \in \mathcal{D}_{n}}\left|h_{i d}\right|^{2}\right),
$$

289 where the subscript ' $b$ ' in $C_{b d}$ denotes the best SR. It is observed 290 from (14) that the legitimate transmission capacity of the SRS 291 scheme is determined by the maximum of independent random 292 variables (RVs) $\left|h_{i d}\right|^{2}$ for different SRs. By contrast, one can 293 see from (4) that the capacity of classic direct transmission is 294 affected by the single RV $\left|h_{s d}\right|^{2}$. If all RVs $\left|h_{i d}\right|^{2}$ and $\left|h_{s d}\right|^{2}$ are 295 independent and identically distributed (i.i.d), it would be most 296 likely that $\max _{i \in \mathcal{D}_{n}}\left|h_{i d}\right|^{2}$ is much higher than $\left|h_{s d}\right|^{2}$ for a sufficiently

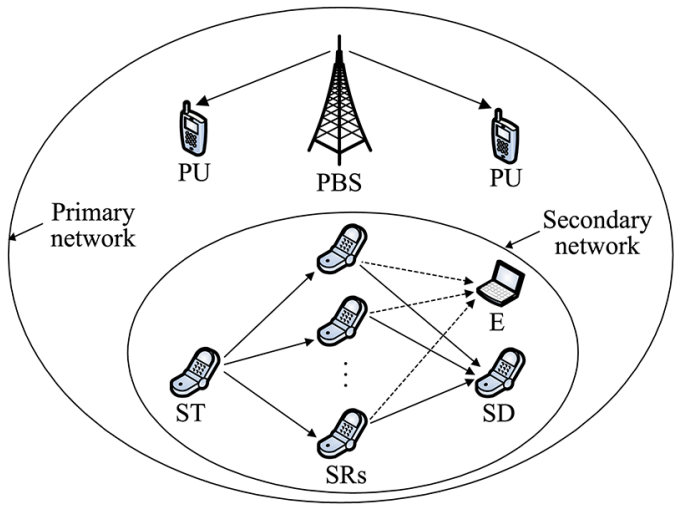

SRs: Secondary Relays

Fig. 2. A cognitive relay network consists of one ST, one SD and $N$ SRs in the presence of an E.

large number of SRs, resulting in a performance improvement 297 for the SRS scheme over the classic direct transmission. How- 298 ever, if the RVs $\left|h_{i d}\right|^{2}$ and $\left|h_{s d}\right|^{2}$ are non-identically distributed 299 and the mean value of $\left|h_{s d}\right|^{2}$ is much higher than that of $\left|h_{i d}\right|^{2}, 300$ then it may be more likely that $\max _{i \in \mathcal{D}_{n}}\left|h_{i d}\right|^{2}$ is smaller than $\left|h_{s d}\right|^{2} 301$ for a given number of SRs. In this extreme case, the classic 302 direct transmission may perform better than the SRS scheme. 303 It is worth mentioning that in practice, the average fading gain 304 of the $\mathrm{SR}_{i}-\mathrm{SD}$ channel, $\left|h_{i d}\right|^{2}$, should not be less than that 305 of the ST-SD channel $\left|h_{s d}\right|^{2}$, since SRs are typically placed 306 in the middle between the ST and SD. Hence, a performance 307 improvement for the SRS scheme over classic direct transmis- 308 sion would be achieved in practical wireless systems. Note 309 that although a factor $1 / 2$ in (14) is imposed on the capacity 310 of the main channel, it would not affect the performance of 311 the SRS scheme from a SRT perspective, since the capacity 312 of the wiretap channel is also multiplied by $1 / 2$ as will be 313 shown in (16).

314

Additionally, given that the selected SR transmits its 315 decoded result $x_{s}$ at a power of $P_{s}$, the signal received at $\mathrm{E}$ is 316 expressed as

$$
y_{e}=h_{b e} \sqrt{P_{s}} x_{s}+h_{p e} \sqrt{\alpha P_{p}} x_{p}+n_{e},
$$

where $h_{b e}$ and $h_{p e}$ represent the fading coefficients of the chan- 318 nel from "best" SR to E and that from PBS to E, respectively. 319 From (15), the capacity of the channel spanning from the "best" 320 $\mathrm{SR}$ to $\mathrm{E}$ is given by

$$
C_{b e}=\frac{1}{2} \log _{2}\left(1+\frac{\left|h_{b e}\right|^{2} \gamma_{s}}{\alpha\left|h_{p e}\right|^{2} \gamma_{p}+1}\right),
$$

where $b \in \mathcal{D}_{n}$ is determined by the relay selection criterion 322 given in (13). As shown in (16), the eavesdropper's channel 323 capacity is affected by the channel state information (CSI) 324 $\left|h_{b e}\right|^{2}$ of the wiretap channel spanning from the "best" relay to 325 the eavesdropper. However, one can see from (13) that the best 326 relay is selected from the decoding set $\mathcal{D}_{n}$ solely based on the 327 main channel's CSI $\left|h_{i d}\right|^{2}$ i.e. without taking into account the 328 eavesdropper's CSI knowledge of $\left|h_{i e}\right|^{2}$. This means that the 329 selection of the best relay aiming for maximizing the legitimate 330 transmission capacity of (14) would not lead to significantly 331 
332 beneficial or adverse impact on the eavesdropper's channel 333 capacity, since the main channel and the wiretap channel are 334 independent of each other.

335 For example, if the random variables (RVs) $\left|h_{i e}\right|^{2}$ related to 336 the different relays are i.i.d, we can readily infer by the law 337 of total probability that $\left|h_{b e}\right|^{2}$ has the same probability den338 sity function (PDF) as $\left|h_{i e}\right|^{2}$, implying that the eavesdropper's 339 channel capacity of (16) is not affected by the selection of the 340 best relay given by (13). Therefore, the SRS scheme has no 341 obvious advantage over the classic direct transmission in terms 342 of minimizing the capacity of the wiretap channel. To elaborate 343 a little further, according to the SRT trade-off, a reduction of 344 the outage probability (OP) due to the capacity enhancement 345 of the main channel achieved by using the selection of the 346 best relay would be converted into an intercept probability 347 (IP) improvement, which will be numerically illustrated in 348 Section IV.

\section{D. Multi-Relay Selection}

350 This subsection presents a MRS scheme, where multiple SRs 351 are employed for simultaneously forwarding the source signal $352 x_{s}$ to SD. To be specific, ST first transmits $x_{s}$ to $N$ SRs over a 353 detected spectrum hole. As mentioned in Subsection II-C, we 354 denote by $\mathcal{D}$ the set of SRs that successfully decode $x_{s}$. If $\mathcal{D}$ 355 is empty, all SRs fail to decode $x_{s}$ and will not forward the 356 source signal, thus both SD and E are unable to decode $x_{s}$. If $357 \mathcal{D}$ is non-empty (i.e. $\mathcal{D}=\mathcal{D}_{n}$ ), all SRs within $\mathcal{D}_{n}$ are utilized 358 for simultaneously transmitting $x_{s}$ to SD. This differs from the 359 SRS scheme, where only a single $\mathrm{SR}$ is chosen from $\mathcal{D}_{n}$ for 360 forwarding $x_{s}$ to SD. To make effective use of multiple SRs, a 361 weight vector denoted by $w=\left[w_{1}, w_{2}, \cdots, w_{\left|\mathcal{D}_{n}\right|}\right]^{T}$ is employed 362 at the SRs for transmitting $x_{s}$, where $\left|\mathcal{D}_{n}\right|$ is the cardinality of 363 the set $\mathcal{D}_{n}$. For the sake of a fair comparison with the SRS 364 scheme in terms of power consumption, the total transmit power 365 across all SRs within $\mathcal{D}_{n}$ shall be constrained to $P_{s}$ and thus the 366 weight vector $w$ should be normalized according to $\|w\|=1$. 367 Thus, given $\mathcal{D}=\mathcal{D}_{n}$ and considering that all SRs within $\mathcal{D}_{n}$ are 368 selected for simultaneously transmitting $x_{s}$ with a weight vector $369 w$, the signal received at SD is expressed as

$$
y_{d}^{\text {multi }}=\sqrt{P_{s}} w^{T} H_{d} x_{s}+\sqrt{\alpha P_{p}} h_{p d} x_{p}+n_{d}
$$

370 where $H_{d}=\left[h_{1 d}, h_{2 d}, \cdots, h_{\left|\mathcal{D}_{n}\right| d}\right]^{T}$. Similarly, the signal received 371 at E can be written as

$$
y_{e}^{\text {multi }}=\sqrt{P_{s}} w^{T} H_{e} x_{s}+\sqrt{\alpha P_{p}} h_{p e} x_{p}+n_{e},
$$

372 where $H_{e}=\left[h_{1 e}, h_{2 e}, \cdots, h_{\left|\mathcal{D}_{n}\right| e}\right]^{T}$. From (17) and (18), the 373 signal-to-interference-plus-noise ratios (SINRs) at SD and E 374 are, respectively, given by

$$
\operatorname{SINR}_{d}^{\text {multi }}=\frac{\gamma_{s}}{\alpha\left|h_{p d}\right|^{2} \gamma_{p}+1}\left|w^{T} H_{d}\right|^{2},
$$

375 and

$$
\operatorname{SINR}_{e}^{\text {multi }}=\frac{\gamma_{s}}{\alpha\left|h_{p e}\right|^{2} \gamma_{p}+1}\left|w^{T} H_{e}\right|^{2}
$$

In this work, the weight vector $w$ is optimized by maximizing 376 the SINR at SD, yielding

$$
\max _{w} \operatorname{SINR}_{d}^{\text {multi }}, \quad \text { s.t. }\|w\|=1
$$

where the constraint is used for normalization purposes. Using 378 the Cauchy-Schwarz inequality [32], we can readily obtain the 379 optimal weight vector $w_{\text {opt }}$ from (21) as

$$
w_{\mathrm{opt}}=\frac{H_{d}^{*}}{\left|H_{d}\right|},
$$

which indicates that the optimal vector design only requires the 381 SR-SD CSI $H_{d}$, whilst dispensing with the eavesdropper's CSI 382 $H_{e}$. Substituting the optimal vector $w_{\text {opt }}$ from (22) into (19) and 383 (20) and using Shannon's capacity formula, we can obtain the 384 channel capacities achieved at both SD and E as

$$
C_{d}^{\text {multi }}=\frac{1}{2} \log _{2}\left(1+\frac{\gamma_{s}}{\alpha \gamma_{p}\left|h_{p d}\right|^{2}+1} \sum_{i \in \mathcal{D}_{n}}\left|h_{i d}\right|^{2}\right),
$$

and

$$
C_{e}^{\text {multi }}=\frac{1}{2} \log _{2}\left(1+\frac{\gamma_{s}}{\alpha \gamma_{p}\left|h_{p e}\right|^{2}+1} \frac{\left|H_{d}^{H} H_{e}\right|^{2}}{\left|H_{d}\right|^{2}}\right),
$$

for $\mathcal{D}=\mathcal{D}_{n}$, where $H$ represents the Hermitian transpose. One 387 can observe from (14) and (23) that the difference between the 388 capacity expressions $C_{b d}$ and $C_{d}^{\text {multi }}$ only lies in the fact that 389 the maximum of RVs $\left|h_{i d}\right|^{2}$ for different SRs (i.e., $\max _{i \in \mathcal{D}_{n}}\left|h_{i d}\right|^{2}$ ) 390 is used for the SRS scheme, while the sum of RVs $\left|h_{i d}\right|^{2} 391$ (i.e., $\sum_{i \in \mathcal{D}_{n}}\left|h_{i d}\right|^{2}$ ) is employed for the MRS scheme. Clearly, 392 we have $\sum_{i \in \mathcal{D}_{n}}\left|h_{i d}\right|^{2}>\max _{i \in \mathcal{D}_{n}}\left|h_{i d}\right|^{2}$, resulting in a performance 393 gain for MRS over SRS in terms of maximizing the legitimate 394 transmission capacity. Moreover, since the main channel $H_{d} 395$ and the wiretap channel $H_{e}$ are independent of each other, the 396 optimal weights assigned for the multiple relays based on $H_{d} 397$ will only slightly affect the eavesdropper's channel capacity. 398 This means that the MRS and SRS schemes achieve more or 399 less the same performance in terms of the capacity of the wire- 400 tap channel. Nevertheless, given a fixed outage requirement, 401 the MRS scheme can achieve a better intercept performance 402 than the SRS scheme, because according to the SRT, an outage 403 reduction achieved by the capacity enhancement of the legiti- 404 mate transmission relying on the MRS would be converted into 405 an intercept improvement. To be specific, given an enhanced 406 capacity of the legitimate transmission, we may increase the 407 data rate $R$ based on the OP definition of (25) for maintaining 408 a fixed OP, which, in turn leads to a reduction of the IP, since a 409 higher data rate would result in a lower IP, according to the IP 410 definition of (26).

411

It needs to be pointed out that in the MRS scheme, a 412 high-complexity symbol-level synchronization is required for 413 multiple distributed SRs, when simultaneously transmitting to 414 SD, whereas the SRS does not require such a complex synchro- 415 nization process. Thus, the performance improvement of MRS 416 over SRS is achieved at the cost of a higher implementation 417 
418 complexity. Additionally, the synchronization imperfections of 419 the MRS scheme will impose a performance degradation, which 420 may even lead to a performance for the MRS scheme becoming 421 worse than that of the SRS scheme.

422 Throughout this paper, the Rayleigh model is used for char423 acterizing the fading amplitudes (e.g., $\left|h_{s d}\right|,\left|h_{s i}\right|,\left|h_{i d}\right|$, etc.) of 424 wireless channels, which, in turn, implies that the fading square 425 magnitudes $\left|h_{s d}\right|^{2},\left|h_{s i}\right|^{2}$ and $\left|h_{i d}\right|^{2}$ are exponentially distributed 426 random variables (RVs). So far, we have completed the presen427 tation of the signal model of the direct transmission, of the SRS, 428 and of the MRS schemes for CR networks applications in the 429 presence of eavesdropping attacks.

\section{III. SRT ANALYSIS OVER RAYLEIGH FADING CHANNELS}

431 This section presents the SRT analysis of the direct transmis432 sion, SRS and MRS schemes over Rayleigh fading channels. 433 As discussed in [31], the security and reliability are quantified 434 in terms of the IP and OP experienced by the eavesdropper and 435 destination, respectively. It is pointed out that in CR networks, 436 ST starts to transmit its signal only when an available spectrum 437 hole is detected. Similarly to [34], the OP and IP are thus 438 calculated under the condition that the licensed spectrum is 439 detected to be unoccupied by the PBS. The following gives the 440 definition of OP and IP.

441 Definition 1: Let $C_{d}$ and $C_{e}$ represent the channel capacities 442 achieved at the destination and eavesdropper, respectively. The $443 \mathrm{OP}$ and IP are, respectively, defined as

$$
P_{\text {out }}=\operatorname{Pr}\left(C_{d}<R \mid \hat{H}=H_{0}\right),
$$

444 and

$$
P_{\text {int }}=\operatorname{Pr}\left(C_{e}>R \mid \hat{H}=H_{0}\right),
$$

445 where $R$ is the data rate.

\section{A. Direct Transmission}

447 Let us first analyze the SRT performance of the conventional 448 direct transmission. Given that a spectrum hole has been de449 tected, the OP of direct transmission is obtained from (25) as

$$
P_{\text {out }}^{\text {direct }}=\operatorname{Pr}\left(C_{s d}<R \mid \hat{H}=H_{0}\right),
$$

450 where $C_{s d}$ is given by (4). Using the law of total probability, we 451 can rewrite $(27)$ as

$$
P_{\text {out }}^{\text {direct }}=\operatorname{Pr}\left(C_{s d}<R, H_{0} \mid \hat{H}=H_{0}\right)+\operatorname{Pr}\left(C_{s d}<R, H_{1} \mid \hat{H}=H_{0}\right),
$$

452 which can be further expressed as

$$
\begin{aligned}
P_{\text {out }}^{\text {direct }}=\operatorname{Pr}( & \left.C_{\text {sd }}<R \mid H_{0}, \hat{H}=H_{0}\right) \operatorname{Pr}\left(H_{0} \mid \hat{H}=H_{0}\right) \\
& +\operatorname{Pr}\left(C_{s d}<R \mid H_{1}, \hat{H}=H_{0}\right) \operatorname{Pr}\left(H_{1} \mid \hat{H}=H_{0}\right) .
\end{aligned}
$$

453 It is shown from (2) that given $H_{0}$ and $H_{1}$, the parameter $\alpha$ is 454 obtained as $\alpha=0$ and $\alpha=1$, respectively. Thus, combining (2) and (4), we have $C_{s d}=\log _{2}\left(1+\left|h_{s d}\right|^{2} \gamma_{s}\right)$ given $H_{0}$ and $C_{s d}=455$ $\log _{2}\left(1+\frac{\left|h_{s d}\right|^{2} \gamma_{s}}{\left|h_{p d}\right|^{2} \gamma_{p}+1}\right)$ given $H_{1}$. Substituting this result into (29) 456 yields

$$
\begin{aligned}
P_{\text {out }}^{\text {direct }}= & \operatorname{Pr}\left(\left|h_{s d}\right|^{2} \gamma_{s}<2^{R}-1\right) \operatorname{Pr}\left(H_{0} \mid \hat{H}=H_{0}\right) \\
& +\operatorname{Pr}\left(\frac{\left|h_{s d}\right|^{2} \gamma_{s}}{\left|h_{p d}\right|^{2} \gamma_{p}+1}<2^{R}-1\right) \operatorname{Pr}\left(H_{1} \mid \hat{H}=H_{0}\right)
\end{aligned}
$$

Moreover, the terms $\operatorname{Pr}\left(H_{0} \mid \hat{H}=H_{0}\right)$ and $\operatorname{Pr}\left(H_{1} \mid \hat{H}=H_{0}\right)$ can be 458 obtained by using Bayes' theorem as

$$
\begin{aligned}
\operatorname{Pr}\left(H_{0} \mid \hat{H}=H_{0}\right) & =\frac{\operatorname{Pr}\left(\hat{H}=H_{0} \mid H_{0}\right) \operatorname{Pr}\left(H_{0}\right)}{\sum_{i \in\{0,1\}} \operatorname{Pr}\left(\hat{H}=H_{0} \mid H_{i}\right) \operatorname{Pr}\left(H_{i}\right)} \\
& =\frac{P_{0}\left(1-P_{f}\right)}{P_{0}\left(1-P_{f}\right)+\left(1-P_{0}\right)\left(1-P_{d}\right)} \triangleq \pi_{0},
\end{aligned}
$$

and

$$
\operatorname{Pr}\left(H_{1} \mid \hat{H}=H_{0}\right)=\frac{\left(1-P_{0}\right)\left(1-P_{d}\right)}{P_{0}\left(1-P_{f}\right)+\left(1-P_{0}\right)\left(1-P_{d}\right)} \triangleq \pi_{1},
$$

where $P_{0}=\operatorname{Pr}\left(H_{0}\right)$ is the probability that the licensed spec- 461 trum band is unoccupied by $\mathrm{PBS}$, while $P_{d}=\operatorname{Pr}\left(\hat{H}=H_{1} \mid H_{1}\right) 462$ and $P_{f}=\operatorname{Pr}\left(\hat{H}=H_{1} \mid H_{0}\right)$ are the SDP and FAP, respectively. 463 For notational convenience, we introduce the shorthand $\pi_{0}=464$ $\operatorname{Pr}\left(H_{0} \mid \hat{H}=H_{0}\right), \pi_{1}=\operatorname{Pr}\left(H_{1} \mid \hat{H}=H_{0}\right)$ and $\Delta=\frac{2^{R}-1}{\gamma_{s}}$. Then, 465 using (31) and (32), we rewrite (30) as 466

$P_{\text {out }}^{\text {direct }}=\pi_{0} \operatorname{Pr}\left(\left|h_{s d}\right|^{2}<\Delta\right)+\pi_{1} \operatorname{Pr}\left(\left|h_{s d}\right|^{2}-\left|h_{p d}\right|^{2} \gamma_{p} \Delta<\Delta\right)$.

Noting that $\left|h_{s d}\right|^{2}$ and $\left|h_{p d}\right|^{2}$ are independently and exponen- 467 tially distributed RVs with respective means of $\sigma_{s d}^{2}$ and $\sigma_{p d}^{2}, 468$ we obtain

$$
\operatorname{Pr}\left(\left|h_{s d}\right|^{2}<\Delta\right)=1-\exp \left(-\frac{\Delta}{\sigma_{s d}^{2}}\right)
$$

and

$$
\operatorname{Pr}\left(\left|h_{s d}\right|^{2}-\left|h_{p d}\right|^{2} \gamma_{p} \Delta<\Delta\right)=1-\frac{\sigma_{s d}^{2}}{\sigma_{p d}^{2} \gamma_{p} \Delta+\sigma_{s d}^{2}} \exp \left(-\frac{\Delta}{\sigma_{s d}^{2}}\right) .
$$

Additionally, we observe from (26) that an intercept event 471 occurs, when the capacity of the ST-E channel becomes higher 472 than the data rate. Thus, given that a spectrum hole has been de- 473 tected (i.e. $\hat{H}=H_{0}$ ), ST starts transmitting its signal to SD and 474 E may overhear the ST-SD transmission. The corresponding IP 475 is given by

$$
P_{\text {int }}^{\text {direct }}=\operatorname{Pr}\left(C_{s e}>R \mid \hat{H}=H_{0}\right),
$$

which can be further expressed as

$$
\begin{aligned}
P_{\text {int }}^{\text {direct }}= & \operatorname{Pr}\left(C_{s e}>R \mid \hat{H}=H_{0}, H_{0}\right) \operatorname{Pr}\left(H_{0} \mid \hat{H}=H_{0}\right) \\
& +\operatorname{Pr}\left(C_{s e}>R \mid \hat{H}=H_{0}, H_{1}\right) \operatorname{Pr}\left(H_{1} \mid \hat{H}=H_{0}\right) \\
= & \pi_{0} \operatorname{Pr}\left(\left|h_{s e}\right|^{2}>\Delta\right)+\pi_{1} \operatorname{Pr}\left(\left|h_{s e}\right|^{2}-\left|h_{p e}\right|^{2} \gamma_{p} \Delta>\Delta\right),
\end{aligned}
$$


478 where the second equality is obtained by using $C_{s e}$ from (5). 479 Noting that RVs $\left|h_{s e}\right|^{2}$ and $\left|h_{p e}\right|^{2}$ are exponentially distributed 480 and independent of each other, we can express the terms $481 \operatorname{Pr}\left(\left|h_{s e}\right|^{2}>\Delta\right)$ and $\operatorname{Pr}\left(\left|h_{s e}\right|^{2}-\left|h_{p e}\right|^{2} \gamma_{p} \Delta>\Delta\right)$ as

$$
\operatorname{Pr}\left(\left|h_{s e}\right|^{2}>\Delta\right)=\exp \left(-\frac{\Delta}{\sigma_{s e}^{2}}\right)
$$

482 and

$$
\operatorname{Pr}\left(\left|h_{s e}\right|^{2}-\left|h_{p e}\right|^{2} \gamma_{p} \Delta>\Delta\right)=\frac{\sigma_{s e}^{2}}{\sigma_{p e}^{2} \gamma_{p} \Delta+\sigma_{s e}^{2}} \exp \left(-\frac{\Delta}{\sigma_{s e}^{2}}\right)
$$

483 where $\sigma_{s e}^{2}$ and $\sigma_{p e}^{2}$ are the expected values of $\mathrm{RVs}\left|h_{s e}\right|^{2}$ and $484\left|h_{p e}\right|^{2}$, respectively.

\section{B. Single-Relay Selection}

486 In this subsection, we present the SRT analysis of the pro487 posed SRS scheme. Given $\hat{H}=H_{0}$, the OP of the cognitive 488 transmission relying on SRS is given by

$$
\begin{aligned}
P_{\text {out }}^{\text {single }}=\operatorname{Pr}\left(C_{b d}<\right. & \left.R, \mathcal{D}=\emptyset \mid \hat{H}=H_{0}\right) \\
& +\sum_{n=1}^{2^{N}-1} \operatorname{Pr}\left(C_{b d}<R, \mathcal{D}=\mathcal{D}_{n} \mid \hat{H}=H_{0}\right),
\end{aligned}
$$

489 where $C_{b d}$ represents the capacity of the channel from the 490 "best" SR to SD. In the case of $\mathcal{D}=\emptyset$, no $\mathrm{SR}$ is chosen to 491 forward the source signal, which leads to $C_{b d}=0$ for $\mathcal{D}=\emptyset$. 492 Substituting this result into (40) gives

$$
\begin{aligned}
P_{\text {out }}^{\text {single }}=\operatorname{Pr}(\mathcal{D}=\emptyset \mid & \left.\mid \hat{H}=H_{0}\right) \\
& +\sum_{n=1}^{2^{N}-1} \operatorname{Pr}\left(C_{b d}<R, \mathcal{D}=\mathcal{D}_{n} \mid \hat{H}=H_{0}\right) .
\end{aligned}
$$

493 Using (2), (9), (10), and (14), we can rewrite (41) as (42), 494 shown at the bottom of the page, where $\Lambda=\frac{2^{2 R}-1}{\gamma_{s}}$. Noting 495 that $\left|h_{s i}\right|^{2}$ and $\left|h_{p i}\right|^{2}$ are independent exponentially distributed random variables with respective means of $\sigma_{s i}^{2}$ and $\sigma_{p i}^{2}$, we have 496

$$
\operatorname{Pr}\left(\left|h_{s i}\right|^{2}<\Lambda\right)=1-\exp \left(-\frac{\Lambda}{\sigma_{s i}^{2}}\right)
$$

and

$\operatorname{Pr}\left(\left|h_{s i}\right|^{2}<\Lambda\left|h_{p i}\right|^{2} \gamma_{p}+\Lambda\right)=1-\frac{\sigma_{s i}^{2}}{\sigma_{p i}^{2} \gamma_{p} \Lambda+\sigma_{s i}^{2}} \exp \left(-\frac{\Lambda}{\sigma_{s i}^{2}}\right)$,

where the terms $\operatorname{Pr}\left(\left|h_{s i}\right|^{2}>\Lambda\right), \operatorname{Pr}\left(\left|h_{s j}\right|^{2}<\Lambda\right)$, and $\operatorname{Pr}\left(\left|h_{s i}\right|^{2}>498\right.$ $\left.\Lambda\left|h_{p i}\right|^{2} \gamma_{p}+\Lambda\right)$ can be similarly determined in closed-form. 499 Moreover, based on Appendix A, we obtain $\operatorname{Pr}\left(\max _{i \in \mathcal{D}_{n}}\left|h_{i d}\right|^{2}<\Lambda\right) 500$ and $\operatorname{Pr}\left(\max _{i \in \mathcal{D}_{n}}\left|h_{i d}\right|^{2}<\Lambda\left|h_{p d}\right|^{2} \gamma_{p}+\Lambda\right)$ as

$$
\operatorname{Pr}\left(\max _{i \in \mathcal{D}_{n}}\left|h_{i d}\right|^{2}<\Lambda\right)=\prod_{i \in \mathcal{D}_{n}}\left[1-\exp \left(-\frac{\Lambda}{\sigma_{i d}^{2}}\right)\right],
$$

and

$$
\begin{aligned}
\operatorname{Pr}\left(\max _{i \in \mathcal{D}_{n}}\left|h_{i d}\right|^{2}<\Lambda\left|h_{p d}\right|^{2} \gamma_{p}+\Lambda\right) \\
=1+\sum_{m=1}^{2\left|\mathcal{D}_{n}\right|}(-1)^{\left|\tilde{\mathcal{D}}_{n}(m)\right|} \exp \left(-\sum_{i \in \tilde{\mathcal{D}}_{n}(m)} \frac{\Lambda}{\sigma_{i d}^{2}}\right) \\
\times\left(1+\sum_{i \in \tilde{\mathcal{D}}_{n}(m)} \frac{\Lambda \gamma_{p} \sigma_{p d}^{2}}{\sigma_{i d}^{2}}\right)^{-1},
\end{aligned}
$$

where $\tilde{D}_{n}(m)$ represents the $m$-th non-empty subset of $\mathcal{D}_{n} .503$ Additionally, the IP of the SRS scheme can be expressed as 504

$$
\begin{aligned}
P_{\text {int }}^{\text {single }}=\operatorname{Pr}\left(C_{b e}>\right. & \left.R, \mathcal{D}=\emptyset \mid \hat{H}=H_{0}\right) \\
& +\sum_{n=1}^{2^{N}-1} \operatorname{Pr}\left(C_{b e}>R, \mathcal{D}=\mathcal{D}_{n} \mid \hat{H}=H_{0}\right),
\end{aligned}
$$

where $C_{b e}$ represents the capacity of the channel spanning from 505 the "best" SR to E. Given $\mathcal{D}=\emptyset$, we have $C_{b e}=0$, since 506 no relay is chosen for forwarding the source signal. Thus, 507

$$
\begin{aligned}
P_{\text {out }}^{\text {single }}= & \pi_{0} \prod_{i=1}^{N} \operatorname{Pr}\left(\left|h_{s i}\right|^{2}<\Lambda\right)+\pi_{1} \prod_{i=1}^{N} \operatorname{Pr}\left(\left|h_{s i}\right|^{2}<\Lambda\left|h_{p i}\right|^{2} \gamma_{p}+\Lambda\right) \\
& +\pi_{0} \sum_{n=1}^{2^{N}-1} \prod_{i \in \mathcal{D}_{n}} \operatorname{Pr}\left(\left|h_{s i}\right|^{2}>\Lambda\right) \prod_{j \in \overline{\mathcal{D}}_{n}} \operatorname{Pr}\left(\left|h_{s j}\right|^{2}<\Lambda\right) \operatorname{Pr}\left(\max _{i \in \mathcal{D}_{n}}\left|h_{i d}\right|^{2}<\Lambda\right) \\
+ & \pi_{1} \sum_{n=1}^{2^{N}-1} \prod_{i \in \mathcal{D}_{n}} \operatorname{Pr}\left(\left|h_{s i}\right|^{2}>\Lambda\left|h_{p i}\right|^{2} \gamma_{p}+\Lambda\right) \prod_{j \in \overline{\mathcal{D}}_{n}} \operatorname{Pr}\left(\left|h_{s j}\right|^{2}<\Lambda\left|h_{p j}\right|^{2} \gamma_{p}+\Lambda\right) \\
& \times \operatorname{Pr}\left(\max _{i \in \mathcal{D}_{n}}\left|h_{i d}\right|^{2}<\Lambda\left|h_{p d}\right|^{2} \gamma_{p}+\Lambda\right)
\end{aligned}
$$


508 substituting this result into (47) and using (2), (9), (10), and 509 (16), we arrive at

$$
\begin{aligned}
& P_{\text {int }}^{\text {single }}=\pi_{0} \sum_{n=1}^{2^{N}-1} \prod_{i \in \mathcal{D}_{n}} \operatorname{Pr}\left(\left|h_{s i}\right|^{2}>\Lambda\right) \prod_{j \in \overline{\mathcal{D}}_{n}} \operatorname{Pr}\left(\left|h_{s j}\right|^{2}<\Lambda\right) \\
& \times \operatorname{Pr}\left(\left|h_{b e}\right|^{2}>\Lambda\right) \\
&+\pi_{1} \sum_{n=1}^{2^{N}-1} \prod_{i \in \mathcal{D}_{n}} \operatorname{Pr}\left(\left|h_{s i}\right|^{2}>\Lambda\left|h_{p i}\right|^{2} \gamma_{p}+\Lambda\right) \\
& \times \prod_{j \in \overline{\mathcal{D}}_{n}} \operatorname{Pr}\left(\left|h_{s j}\right|^{2}<\Lambda\left|h_{p j}\right|^{2} \gamma_{p}+\Lambda\right) \\
& \times \operatorname{Pr}\left(\left|h_{b e}\right|^{2}>\Lambda\left|h_{p e}\right|^{2} \gamma_{p}+\Lambda\right)
\end{aligned}
$$

510 where the closed-form expressions of $\operatorname{Pr}\left(\left|h_{s i}\right|^{2}>\Lambda\right)$ and $511 \operatorname{Pr}\left(\left|h_{s i}\right|^{2}>\Lambda\left|h_{p i}\right|^{2} \gamma_{p}+\Lambda\right)$ can be readily obtained by using 512 (43) and (44). Using the results in Appendix B, we can express $513 \operatorname{Pr}\left(\left|h_{b e}\right|^{2}>\Lambda\right)$ and $\operatorname{Pr}\left(\left|h_{b e}\right|^{2}>\Lambda\left|h_{p e}\right|^{2} \gamma_{p}+\Lambda\right)$ as

$$
\begin{aligned}
& \operatorname{Pr}\left(\left|h_{b e}\right|^{2}>\Lambda\right)=\sum_{i \in \mathcal{D}_{n}} \exp \left(-\frac{\Lambda}{\sigma_{i e}^{2}}\right) \\
& \times\left[1+\sum_{m=1}^{{ }^{\left|\mathcal{D}_{n}\right|-1}-1}(-1)^{\left|C_{n}(m)\right|}\left(1+\sum_{j \in C_{n}(m)} \frac{\sigma_{i d}^{2}}{\sigma_{j d}^{2}}\right)^{-1}\right],
\end{aligned}
$$

514 and

$$
\begin{gathered}
\operatorname{Pr}\left(\left|h_{b e}\right|^{2}>\Lambda\left|h_{p e}\right|^{2} \gamma_{p}+\Lambda\right)=\sum_{i \in \mathcal{D}_{n}} \frac{\sigma_{i e}^{2}}{\sigma_{p e}^{2} \gamma_{p} \Lambda+\sigma_{i e}^{2}} \exp \left(-\frac{\Lambda}{\sigma_{i e}^{2}}\right) \\
\times\left[1+\sum_{m=1}^{{ }^{\left|\mathcal{D}_{n}\right|-1}-1}(-1)^{\left|C_{n}(m)\right|}\left(1+\sum_{j \in C_{n}(m)} \frac{\sigma_{i d}^{2}}{\sigma_{j d}^{2}}\right)^{-1}\right],
\end{gathered}
$$

515 where $C_{n}(m)$ represents the $m$-th non-empty subset of $\mathcal{D}_{n}-\{i\}$ 516 and ' - ' represents the set difference.

\section{C. Multi-Relay Selection}

518 This subsection analyzes the SRT of our MRS scheme for 519 transmission over Rayleigh fading channels. Similarly to (41), the $\mathrm{OP}$ in this case is given by

$$
\begin{aligned}
P_{\text {out }}^{\text {mult }}=\operatorname{Pr}(\mathcal{D} & \left.=\emptyset \mid \hat{H}=H_{0}\right) \\
& +\sum_{n=1}^{2^{N}-1} \operatorname{Pr}\left(C_{d}^{\text {multi }}<R, \mathcal{D}=\mathcal{D}_{n} \mid \hat{H}=H_{0}\right) .
\end{aligned}
$$

Using (2), (9), (10) and (23), we can rewrite (51) as (52), shown 521 at the bottom of the page, where the closed-form expressions 522 of $\operatorname{Pr}\left(\left|h_{s i}\right|^{2}<\Lambda\right), \operatorname{Pr}\left(\left|h_{s i}\right|^{2}<\Lambda\left|h_{p i}\right|^{2} \gamma_{p}+\Lambda\right), \operatorname{Pr}\left(\left|h_{s i}\right|^{2}>\Lambda\right), 523$ $\operatorname{Pr}\left(\left|h_{s j}\right|^{2}<\Lambda\right)$ and $\operatorname{Pr}\left(\left|h_{s i}\right|^{2}>\Lambda\left|h_{p i}\right|^{2} \gamma_{p}+\Lambda\right)$ can be readily 524 derived, as shown in (43) and (44). However, it is challenging 525 to obtain the closed-form expressions of $\operatorname{Pr}\left(\sum_{i \in \mathcal{D}_{1}}\left|h_{i d}\right|^{2}<\Lambda\right)$ and 526 $\operatorname{Pr}\left(\sum_{i \in \mathcal{D}_{n}}\left|h_{i d}\right|^{2}<\gamma_{p} \Lambda\left|h_{p d}\right|^{2}+\Lambda\right)$. For simplicity, we assume that 527 the fading coefficients of all SRs-SD channels, i.e. $\left|h_{i d}\right|^{2}$ for 528 $i \in\{1,2, \cdots, N\}$, are i.i.d. RVs having the same mean (average 529 channel gain) denoted by $\sigma_{d}^{2}=E\left(\left|h_{i d}\right|^{2}\right)$. This assumption is 530 widely used in the cooperative relaying literature and it is 531 valid in a statistical sense, provided that all SRs are uniformly 532 distributed over a certain geographical area. Assuming that 533 RVs of $\left|h_{i d}\right|^{2}$ for $i \in \mathcal{D}_{n}$ are i.i.d., based on Appendix C, 534 we arrive at

$$
\operatorname{Pr}\left(\sum_{i \in \mathcal{D}_{n}}\left|h_{i d}\right|^{2}<\Lambda\right)=\Gamma\left(\frac{\Lambda}{\sigma_{d}^{2}},\left|\mathcal{D}_{n}\right|\right),
$$

$$
\begin{aligned}
& \text { and } \\
& \begin{array}{r}
\operatorname{Pr}\left(\sum_{i \in \mathcal{D}_{n}}\left|h_{i d}\right|^{2}<\gamma_{p} \Lambda\left|h_{p d}\right|^{2}+\Lambda\right)=\Gamma\left(\frac{\Lambda}{\sigma_{d}^{2}},\left|\mathcal{D}_{n}\right|\right) \\
+\frac{\left[1-\Gamma\left(\Lambda \sigma_{d}^{-2}+\sigma_{p d}^{-2} \gamma_{p}^{-1},\left|\mathcal{D}_{n}\right|\right)\right]}{\left(1+\sigma_{d}^{2} \sigma_{p d}^{-2} \gamma_{p}^{-1} \Lambda^{-1}\right)^{\left|\mathcal{D}_{n}\right|}} e^{1 /\left(\sigma_{p d}^{2} \gamma_{p}\right)}
\end{array}
\end{aligned}
$$

where $\Gamma(x, k)=\int_{0}^{x} \frac{t^{k-1}}{\Gamma(k)} e^{-t} d t$ is known as the incomplete 537 Gamma function [32]. Substituting (53) and (54) into (52) 538 yields a closed-form OP expression for the proposed MRS 539 scheme.

$$
\begin{aligned}
P_{\text {out }}^{\text {multi }}= & \pi_{0} \prod_{i=1}^{N} \operatorname{Pr}\left(\left|h_{s i}\right|^{2}<\Lambda\right)+\pi_{1} \prod_{i=1}^{N} \operatorname{Pr}\left(\left|h_{s i}\right|^{2}<\Lambda\left|h_{p i}\right|^{2} \gamma_{p}+\Lambda\right) \\
+ & \pi_{0} \sum_{n=1}^{2^{N}-1} \prod_{i \in \mathcal{D}_{n}} \operatorname{Pr}\left(\left|h_{s i}\right|^{2}>\Lambda\right) \prod_{j \in \overline{\mathcal{D}}_{n}} \operatorname{Pr}\left(\left|h_{s j}\right|^{2}<\Lambda\right) \operatorname{Pr}\left(\sum_{i \in \mathcal{D}_{n}}\left|h_{i d}\right|^{2}<\Lambda\right) \\
+ & \pi_{1} \sum_{n=1}^{2^{N}-1} \prod_{i \in \mathcal{D}_{n}} \operatorname{Pr}\left(\left|h_{s i}\right|^{2}>\Lambda\left|h_{p i}\right|^{2} \gamma_{p}+\Lambda\right) \prod_{j \in \overline{\mathcal{D}}_{n}} \operatorname{Pr}\left(\left|h_{s j}\right|^{2}<\Lambda\left|h_{p j}\right|^{2} \gamma_{p}+\Lambda\right) \\
& \times \operatorname{Pr}\left(\sum_{i \in \mathcal{D}_{n}}\left|h_{i d}\right|^{2}<\gamma_{p} \Lambda\left|h_{p d}\right|^{2}+\Lambda\right)
\end{aligned}
$$


541 Next, we present the IP analysis of the MRS scheme. Simi542 larly to (48), the IP of the MRS can be obtained from (24) as

$$
\begin{aligned}
P_{\text {int }}^{\text {multi }}=\pi_{0} \sum_{n=1}^{2^{N}-1} \prod_{i \in \mathcal{D}_{n}} \operatorname{Pr}\left(\left|h_{s i}\right|^{2}>\Lambda\right) \prod_{j \in \overline{\mathcal{D}}_{n}} \operatorname{Pr}\left(\left|h_{s j}\right|^{2}<\Lambda\right) \\
\quad \times \operatorname{Pr}\left(\frac{\left|H_{d}^{H} H_{e}\right|^{2}}{\left|H_{d}\right|^{2}}>\Lambda\right) \\
+\pi_{1} \sum_{n=1}^{2^{N}-1} \prod_{i \in \mathcal{D}_{n}} \operatorname{Pr}\left(\left|h_{s i}\right|^{2}>\Lambda\left|h_{p i}\right|^{2} \gamma_{p}+\Lambda\right) \\
\times \prod_{j \in \overline{\mathcal{D}}_{n}} \operatorname{Pr}\left(\left|h_{s j}\right|^{2}<\Lambda\left|h_{p j}\right|^{2} \gamma_{p}+\Lambda\right) \\
\quad \times \operatorname{Pr}\left(\frac{\left|H_{d}^{H} H_{e}\right|^{2}}{\left|H_{d}\right|^{2}}>\gamma_{p} \Lambda\left|h_{p e}\right|^{2}+\Lambda\right),
\end{aligned}
$$

543 where the closed-form expressions of $\operatorname{Pr}\left(\left|h_{s i}\right|^{2}>\Lambda\right)$, $544 \operatorname{Pr}\left(\left|h_{s j}\right|^{2}<\Lambda\right), \operatorname{Pr}\left(\left|h_{s i}\right|^{2}>\Lambda\left|h_{p i}\right|^{2} \gamma_{p}+\Lambda\right)$ and $\operatorname{Pr}\left(\left|h_{s j}\right|^{2}<\right.$ $545 \Lambda\left|h_{p j}\right|^{2} \gamma_{p}+\Lambda$ ) may be readily derived by using (43) and (44). 546 However, it is challenging to obtain the closed-form solutions 547 for $\operatorname{Pr}\left(\frac{\left|H_{d}^{H} H_{e}\right|^{2}}{\left|H_{d}\right|^{2}}>\Lambda\right)$ and $\operatorname{Pr}\left(\frac{\left|H_{d}^{H} H_{e}\right|^{2}}{\left|H_{d}\right|^{2}}>\gamma_{p} \Lambda\left|h_{p e}\right|^{2}+\Lambda\right)$. 548 Although finding a general closed-form IP expression for the 549 MRS scheme is challenging, we can obtain the numerical IP 550 results with the aid of computer simulations.

\section{NumericAl RESUlts AND Discussions}

552 In this section, we present our performance comparisons 553 among the direct transmission, the SRS and MRS schemes 554 in terms of their SRT. To be specific, the analytic IP versus 555 OP of the three schemes are obtained by plotting (33), (37), 556 (42), (48), (52), and (55). The simulated IP and OP results of 557 the three schemes are also given to verify the correctness of 558 the theoretical SRT analysis. In our computer simulations, the 559 fading amplitudes (e.g., $\left|h_{s d}\right|,\left|h_{s i}\right|,\left|h_{i d}\right|$, etc.) are first generated 560 based on the Rayleigh distribution having different variances 561 for different channels. Then, the randomly generated fading 562 amplitudes are substituted into the definition of an outage (or 563 intercept) event, which would determine whether an outage (or 564 intercept) event occurs or not. By repeatedly achieving this pro565 cess, we can calculate the relative frequency of occurrence for 566 an outage (intercept) event, which is the simulated OP (or IP). 567 Additionally, the SDP $P_{d}$ and FAP $P_{f}$ are set to $P_{d}=0.99$ 568 and $P_{f}=0.01$, unless otherwise stated. The primary signal569 to-noise ratio (SNR) of $\gamma_{p}=10 \mathrm{~dB}$ and the data rate of $570 R=1 \mathrm{bit} / \mathrm{s} / \mathrm{Hz}$ are used in our numerical evaluations.

571 The artificial noise based method [35], [36] is also consid572 ered for the purpose of numerical comparison with the relay 573 selection schemes. To be specific, in the artificial noise based 574 scheme, ST directly transmits its signal $x_{s}$ to SD, while $N$ SRs 575 attempt to confuse the eavesdropper by sending an interfering 576 signal (referred to as artificial noise) that is approximately 577 designed to lie in the null-space of the legitimate main channel. 578 In this way, the artificial noise will impose interference on the 579 eavesdropper without affecting the SD. For a fair comparison, 580 the total transmit power of the desired signal $x_{s}$ and the artificial 581 noise are constrained to $P_{S}$. Moreover, the equal power alloca582 tion method [35] is used in the numerical evaluation.

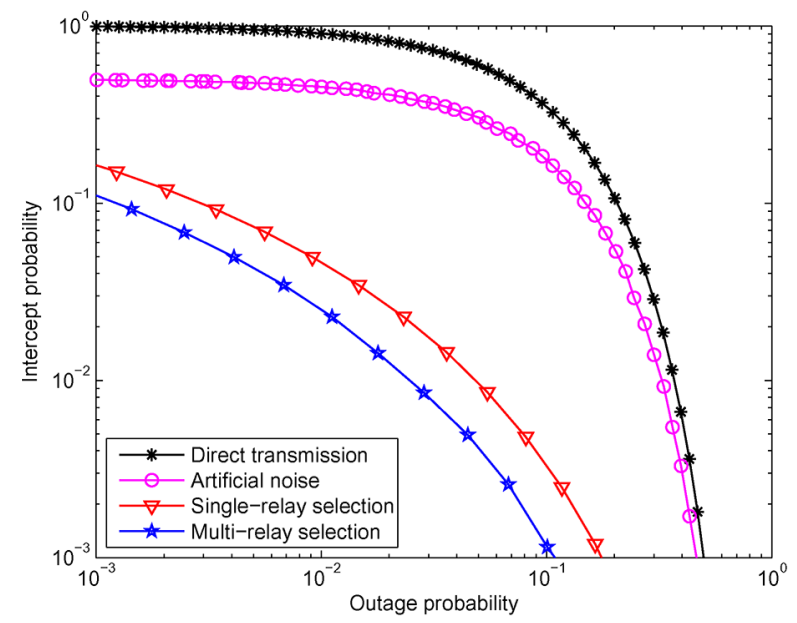

Fig. 3. IP versus OP of the direct transmission, the SRS and the MRS schemes for different $P_{0}$ with $P_{0}=0.8, \gamma_{s} \in[0,35 \mathrm{~dB}], N=6, \sigma_{s d}^{2}=\sigma_{s i}^{2}=\sigma_{i d}^{2}=1$, $\sigma_{s e}^{2}=\sigma_{i e}^{2}=0.1$, and $\sigma_{p d}^{2}=\sigma_{p e}^{2}=\sigma_{p i}^{2}=0.2$.

Fig. 3 shows the IP versus OP of the direct transmission, 583 as well as the SRS and MRS schemes for $P_{0}=0.8$, where 584 the solid lines and discrete marker symbols represent the an- 585 alytic and simulated results, respectively. It can be seen from 586 Fig. 3 that the IP of the direct transmission, the artificial noise 587 based as well as of the proposed SRS and MRS schemes all 588 improve upon tolerating a higher OP, implying that a trade-off 589 exists between the IP (security) and the OP (reliability) of CR 590 transmissions. Fig. 3 also shows that both the proposed SRS 591 and MRS schemes outperform the direct transmission and the 592 artificial noise based approaches in terms of their SRT, showing 593 the advantage of exploiting relay selection against the eaves- 594 dropping attack. Moreover, the SRT performance of the MRS is 595 better than that of the SRS. Although the MRS achieves a better 596 SRT performance than its SRS-aided counterpart, this result 597 is obtained at the cost of a higher implementation complexity, 598 since multiple SRs require high-complexity symbol-level syn- 599 chronization for simultaneously transmitting to the SD, whereas 600 the SRS does not require such elaborate synchronization. 601

Fig. 4 illustrates our numerical SRT comparison between the 602 SRS and MRS schemes for $P_{0}=0.2$ and $P_{0}=0.8$. Observe 603 from Fig. 4 that the MRS scheme performs better than the SRS 604 in terms of its SRT performance for both $P_{0}=0.2$ and $P_{0}=0.8 .605$ It is also seen from Fig. 4 that as $P_{0}$ increases from 0.2 to 606 0.8, the SRT of both the SRS and MRS schemes improves. 607 This is because upon increasing $P_{0}$, the licensed band becomes 608 unoccupied by the PUs with a higher probability and hence the 609 secondary users (SUs) have more opportunities for accessing 610 the licensed band for their data transmissions, which leads 611 to a reduction of the OP for CR transmissions. Meanwhile, 612 increasing $P_{0}$ may simultaneously result in an increase of the IP, 613 since the eavesdropper also has more opportunities for tapping 614 the cognitive transmissions. However, in both the SRS and 615 MRS schemes, the relay selection is performed for the sake 616 of maximizing the legitimate transmission capacity without 617 affecting the eavesdropper's channel capacity. Hence, upon 618 increasing $P_{0}$, it becomes more likely that the reduction of OP 619 is more significant than the increase of IP, hence leading to an 620 overall SRT improvement for the SRS and MRS schemes. 


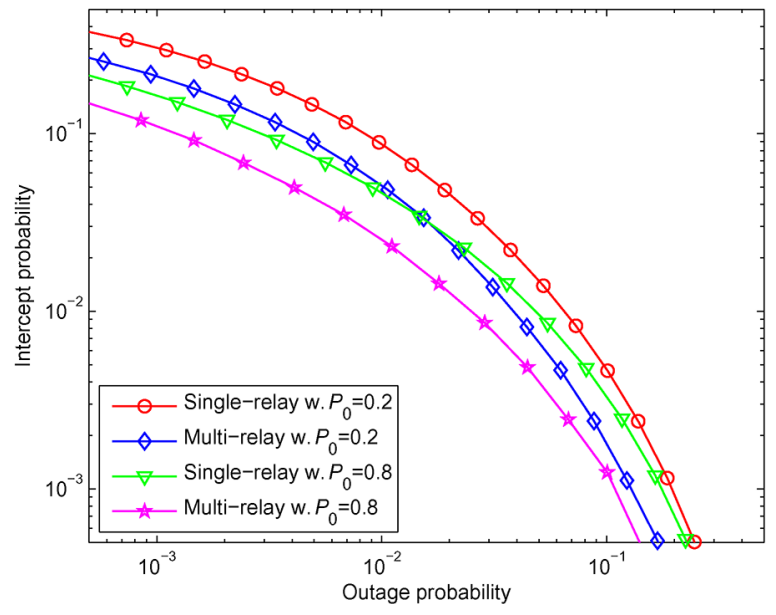

Fig. 4. IP versus OP of the SRS and MRS schemes for different $P_{0}$ with $\gamma_{s} \in[0,30 \mathrm{~dB}], N=6, \sigma_{s d}^{2}=\sigma_{s i}^{2}=\sigma_{i d}^{2}=1, \sigma_{s e}^{2}=\sigma_{i e}^{2}=0.1$, and $\sigma_{p d}^{2}=\sigma_{p e}^{2}=$ $\sigma_{p i}^{2}=0.2$.

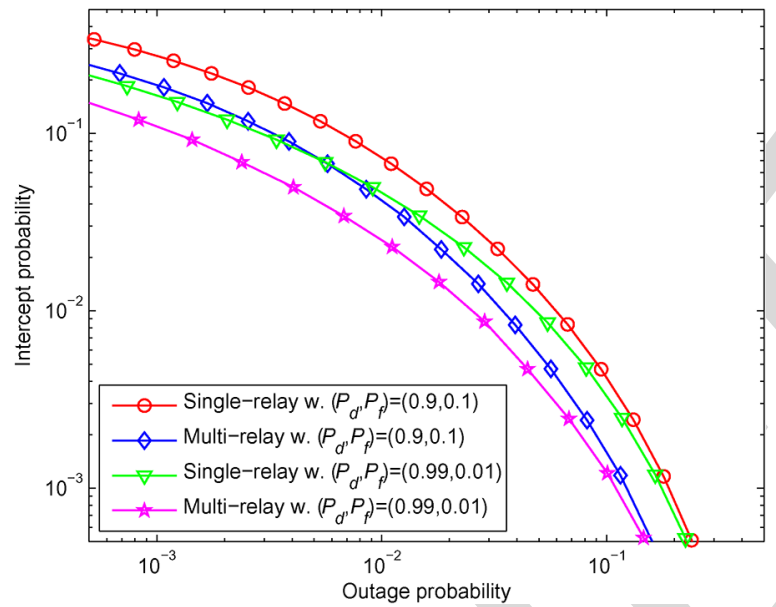

Fig. 5. IP versus OP of the SRS and the MRS schemes for different $\left(P_{d}, P_{f}\right)$ with $P_{0}=0.8, \gamma_{s} \in[0,30 \mathrm{~dB}], N=6, \sigma_{s d}^{2}=\sigma_{s i}^{2}=\sigma_{i d}^{2}=1, \sigma_{s e}^{2}=\sigma_{i e}^{2}=0.1$, and $\sigma_{p d}^{2}=\sigma_{p e}^{2}=\sigma_{p i}^{2}=0.2$.

622 In Fig. 5, we depict the IP versus OP of the SRS and MRS 623 schemes for different spectrum sensing reliabilities, where $624\left(P_{d}, P_{f}\right)=(0.9,0.1)$ and $\left(P_{d}, P_{f}\right)=(0.99,0.01)$ are considered. $625 \mathrm{It}$ is observed that as the spectrum sensing reliability is im626 proved from $\left(P_{d}, P_{f}\right)=(0.9,0.1)$ to $\left(P_{d}, P_{f}\right)=(0.99,0.01)$, the 627 SRTs of the SRS and MRS schemes improve accordingly. This 628 is due to the fact that for an improved sensing reliability, an 629 unoccupied licensed band would be detected more accurately 630 and hence less mutual interference occurs between the PUs 631 and SUs, which results in a better SRT for the secondary 632 transmissions. Fig. 5 also shows that for $\left(P_{d}, P_{f}\right)=(0.9,0.1)$ 633 and $\left(P_{d}, P_{f}\right)=(0.99,0.01)$, the MRS approach outperforms the 634 SRS scheme in terms of the SRT, which further confirms the ad635 vantage of the MRS for protecting the secondary transmissions 636 against eavesdropping attacks.

637 Fig. 6 shows the IP versus OP of the conventional direct 638 transmission as well as of the proposed SRS and MRS schemes 639 for $N=2, N=4$, and $N=8$. It is seen from Fig. 6 that the SRTs

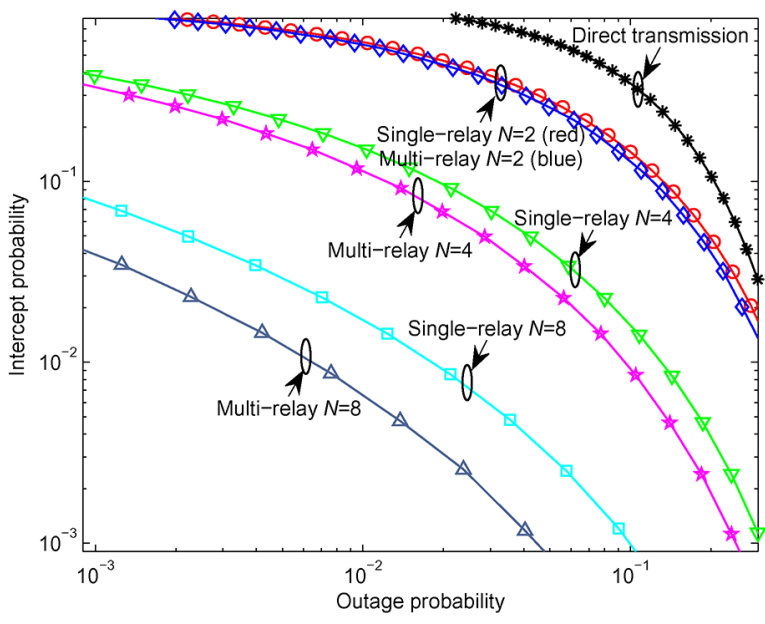

Fig. 6. IP versus $\mathrm{OP}$ of the direct transmission, the $\mathrm{SRS}$ and the MRS schemes for different $N$ with $P_{0}=0.8, \gamma_{s} \in[0,30 \mathrm{~dB}], \sigma_{s d}^{2}=\sigma_{s i}^{2}=\sigma_{i d}^{2}=1, \sigma_{s e}^{2}=\sigma_{i e}^{2}=$ 0.1 , and $\sigma_{p d}^{2}=\sigma_{p e}^{2}=\sigma_{p i}^{2}=0.2$.

of the proposed SRS and MRS schemes are generally better 640 than that of the conventional direct transmission for $N=2,641$ $N=4$ and $N=8$. Moreover, as the number of SRs increases 642 from $N=2$ to 8 , the SRT of the SRS and MRS schemes 643 significantly improves, explicitly demonstrating the security 644 and reliability benefits of exploiting multiple SRs for assisting 645 the secondary transmissions. In other words, the security and 646 reliability of the secondary transmissions can be concurrently 647 improved by increasing the number of SRs. Additionally, as 648 shown in Fig. 6, upon increasing the number of SRs from 649 $N=2$ to 8 , the SRT improvement of MRS over SRS becomes 650 more notable. Again, the SRT advantage of the MRS over the 651 SRS comes at the expense of requiring elaborate symbol-level 652 synchronization among the multiple SRs for simultaneously 653 transmitting to the SD.

\section{CONCLUSION}

In this paper, we proposed relay selection schemes for 656 a CR network consisting of a ST, a SD and multiple SRs 657 communicating in the presence of an eavesdropper. We ex- 658 amined the SRT performance of the SRS and MRS assisted 659 secondary transmissions in the presence of realistic spectrum 660 sensing, where both the security and reliability of secondary 661 transmissions are characterized in terms of their IP and OP, 662 respectively. We also analyzed the SRT of the conventional 663 direct transmission as a benchmark. It was illustrated that as the 664 spectrum sensing reliability increases, the SRTs of both the SRS 665 and MRS schemes improve. We also showed that the proposed 666 SRS and MRS schemes generally outperform the conventional 667 direct transmission and artificial noise based approaches in 668 terms of their SRT. Moreover, the SRT performance of MRS 669 is better than that of SRS. Additionally, as the number of SRs 670 increases, the SRTs of both the SRS and of the MRS schemes 671 improve significantly, demonstrating their benefits in terms 672 of enhancing both the security and reliability of secondary 673 transmissions. 
677 Letting $\left|h_{i d}\right|^{2}=x_{i}$ and $\left|h_{p d}\right|^{2}=y$, the left hand side of (45) 678 and (46) can be rewritten as $\operatorname{Pr}\left(\max _{i \in \mathcal{D}_{n}} x_{i}<\Lambda\right)$ and $\operatorname{Pr}\left(\max _{i \in \mathcal{D}_{n}} x_{i}<\right.$ $\left.679 \Lambda \gamma_{p} y+\Lambda\right)$, respectively. Noting that random variables $\left|h_{i d}\right|^{2}$ and $680\left|h_{p d}\right|^{2}$ are exponentially distributed with respective means $\sigma_{i d}^{2}$ 681 and $\sigma_{p d}^{2}$, and independent of each other, we obtain

$$
\begin{aligned}
\operatorname{Pr}\left(\max _{i \in \mathcal{D}_{n}} x_{i}<\Lambda\right) & =\prod_{i \in \mathcal{D}_{n}} \operatorname{Pr}\left(\left|h_{i d}\right|^{2}<\Lambda\right) \\
& =\prod_{i \in \mathcal{D}_{n}}\left[1-\exp \left(-\frac{\Lambda}{\sigma_{i d}^{2}}\right)\right],
\end{aligned}
$$

682 which is (45). Similarly, the term $\operatorname{Pr}\left(\max _{i \in \mathcal{D}_{n}} x_{i}<\Lambda \gamma_{p} y+\Lambda\right)$ can be 683 computed as

$$
\begin{aligned}
& \operatorname{Pr}\left(\max _{i \in \mathcal{D}_{n}} x_{i}<\Lambda \gamma_{p} y+\Lambda\right) \\
& \quad=\int_{0}^{\infty} \frac{1}{\sigma_{p d}^{2}} \exp \left(-\frac{y}{\sigma_{p d}^{2}}\right) \prod_{i \in \mathcal{D}_{n}}\left(1-\exp \left(-\frac{\Lambda \gamma_{p} y+\Lambda}{\sigma_{i d}^{2}}\right)\right) d y
\end{aligned}
$$

684 wherein $\prod_{i \in \mathcal{D}_{n}}\left(1-\exp \left(-\frac{\Lambda \gamma_{p} y+\Lambda}{\sigma_{i d}^{2}}\right)\right)$ can be further expanded 685 as

$$
\begin{aligned}
\prod_{i \in \mathcal{D}_{n}}\left(1-\exp \left(-\frac{\Lambda \gamma_{p} y+\Lambda}{\sigma_{i d}^{2}}\right)\right)=1 & \\
& +\sum_{m=1}^{2^{\left|\mathcal{D}_{n}\right|}-1}(-1)^{\left|\tilde{\mathcal{D}}_{n}(m)\right|} \exp \left(-\sum_{i \in \tilde{\mathcal{D}}_{n}(m)} \frac{\Lambda \gamma_{p} y+\Lambda}{\sigma_{i d}^{2}}\right),
\end{aligned}
$$

686 where $\left|\mathcal{D}_{n}\right|$ is the cardinality of set $\mathcal{D}_{n}, \tilde{\mathcal{D}}_{n}(m)$ represents the $687 \mathrm{~m}$-th non-empty subset of $\mathcal{D}_{n}$, and $\left|\tilde{\mathcal{D}}_{n}(m)\right|$ is the cardinality 688 of set $\tilde{\mathcal{D}}_{n}(m)$. Substituting $\prod_{i \in \mathcal{D}_{n}}\left(1-\exp \left(-\frac{\Lambda \gamma_{p} y+\Lambda}{\sigma_{i d}^{2}}\right)\right)$ from 689 (A.3) into (A.2) yields

$$
\begin{aligned}
& \operatorname{Pr}\left(\max _{i \in \mathcal{D}_{n}} x_{i}<\Lambda \gamma_{p} y+\Lambda\right)=\int_{0}^{\infty} \frac{1}{\sigma_{p d}^{2}} \exp \left(-\frac{y}{\sigma_{p d}^{2}}\right) d y \\
& +\sum_{m=1}^{2\left|\mathcal{D}_{n}\right|}(-1)^{\left|\tilde{D}_{n}(m)\right|} \frac{1}{\sigma_{p d}^{2}} \\
& \quad \times \int_{0}^{\infty} \exp \left(-\frac{y}{\sigma_{p d}^{2}}-\sum_{i \in \tilde{\mathcal{D}}_{n}(m)} \frac{\Lambda \gamma_{p} y+\Lambda}{\sigma_{i d}^{2}}\right) d y
\end{aligned}
$$

Finally, performing the integration of (A.4) yields

$$
\begin{aligned}
& \operatorname{Pr}\left(\max _{i \in \mathcal{D}_{n}} x_{i}<\Lambda \gamma_{p} y+\Lambda\right)=1 \\
& \quad+\sum_{m=1}^{2\left|\mathcal{D}_{n}\right|}-1 \\
& \quad \times\left(1+\sum_{i \in \tilde{\mathcal{D}}_{n}(m)} \frac{\Lambda \gamma_{p} \sigma_{p d}^{2}}{\sigma_{i d}^{2}}\right)^{-1} . \tilde{\mathcal{D}}_{n}(m) \mid \exp \left(-\tilde{\mathcal{D}}_{n}(m)\right. \\
& \quad
\end{aligned}
$$

This completes the proof of (45) and (46).

Given $\mathcal{D}=\mathcal{D}_{n}$, any $\mathrm{SR}$ within $\mathcal{D}_{n}$ can be selected as the 694 "best" relay for forwarding the source signal. Thus, using the 695 law of total probability, we have

$$
\begin{aligned}
& \operatorname{Pr}\left(\left|h_{b e}\right|^{2}>\Lambda\right)=\sum_{i \in \mathcal{D}_{n}} \operatorname{Pr}\left(\left|h_{i e}\right|^{2}>\Lambda, b=i\right) \\
& =\sum_{i \in \mathcal{D}_{n}} \operatorname{Pr}\left(\left|h_{i e}\right|^{2}>\Lambda,\left|h_{i d}\right|^{2}>\max _{j \in \mathcal{D}_{n}-\{i\}}\left|h_{j d}\right|^{2}\right) \\
& =\sum_{i \in \mathcal{D}_{n}} \operatorname{Pr}\left(\left|h_{i e}\right|^{2}>\Lambda\right) \operatorname{Pr}\left(\max _{j \in \mathcal{D}_{n}-\{i\}}\left|h_{j d}\right|^{2}<\left|h_{i d}\right|^{2}\right),
\end{aligned}
$$

where in the first line, variable ' $b$ ' stands for the best SR and 697 the second equality is obtained from (13) and '-' represents the 698 set difference. Noting that $\left|h_{i e}\right|^{2}$ is an exponentially distributed 699 random variable with a mean of $\sigma_{i e}^{2}$, we obtain

$$
\operatorname{Pr}\left(\left|h_{i e}\right|^{2}>\Lambda\right)=\exp \left(-\frac{\Lambda}{\sigma_{i e}^{2}}\right) .
$$

Letting $\left|h_{j d}\right|^{2}=x_{j}$ and $\left|h_{i d}\right|^{2}=y$, we have

$$
\begin{aligned}
& \operatorname{Pr}\left(\max _{j \in \mathcal{D}_{n}-\{i\}}\left|h_{j d}\right|^{2}<\left|h_{i d}\right|^{2}\right) \\
& \quad=\int_{0}^{\infty} \frac{1}{\sigma_{i d}^{2}} \exp \left(-\frac{y}{\sigma_{i d}^{2}}\right) \prod_{j \in \mathcal{D}_{n}-\{i\}}\left(1-\exp \left(-\frac{y}{\sigma_{j d}^{2}}\right)\right) d y,
\end{aligned}
$$

$$
\begin{aligned}
& \text { wherein } \prod_{j \in \mathcal{D}_{n}-\{i\}}\left(1-\exp \left(-\frac{y}{\sigma_{j d}^{2}}\right)\right) \text { is expanded by } \\
& \prod_{j \in \mathcal{D}_{n}-\{i\}}\left(1-\exp \left(-\frac{y}{\sigma_{j d}^{2}}\right)\right)=1 \\
& +\sum_{m=1}^{2^{\left|\mathcal{D}_{n}\right|-1}-1}(-1)^{\left|\mathcal{C}_{n}(m)\right|} \exp \left(-\sum_{j \in \mathcal{C}_{n}(m)} \frac{y}{\sigma_{j d}^{2}}\right),
\end{aligned}
$$

where $\left|\mathcal{D}_{n}\right|$ denotes the cardinality of the set $\mathcal{D}_{n}$ and $\mathcal{C}_{n}(m) 703$ represents the $m$-th non-empty subset of " $\mathcal{D}_{n}-\{i\}$ ". Combining 704 (B.3) and (B.4), we obtain

$$
\begin{aligned}
& \operatorname{Pr}\left(\max _{j \in \mathcal{D}_{n}-\{i\}}\left|h_{j d}\right|^{2}<\left|h_{i d}\right|^{2}\right)=1 \\
& \quad+\sum_{m=1}^{2^{\left|\mathcal{D}_{n}\right|-1}-1}(-1)^{\left|\mathcal{C}_{n}(m)\right|}\left(1+\sum_{j \in \mathcal{C}_{n}(m)} \frac{\sigma_{i d}^{2}}{\sigma_{j d}^{2}}\right)^{-1} .
\end{aligned}
$$


706 Substituting (B.2) and (B.5) into (B.1) gives (B.6), shown at 707 the bottom of the page, which is (49). Similarly to (B.1), we 708 can rewrite $\operatorname{Pr}\left(\left|h_{b e}\right|^{2}>\Lambda\left|h_{p e}\right|^{2} \gamma_{p}+\Lambda\right)$ as

$$
\begin{aligned}
& \operatorname{Pr}\left(\left|h_{b e}\right|^{2}>\Lambda\left|h_{p e}\right|^{2} \gamma_{p}+\Lambda\right) \\
& =\sum_{i \in \mathcal{D}_{n}} \operatorname{Pr}\left(\left|h_{i e}\right|^{2}>\Lambda\left|h_{p e}\right|^{2} \gamma_{p}+\Lambda\right) \\
& \quad \times \operatorname{Pr}\left(\max _{j \in\left\{\mathcal{D}_{n}-i\right\}}\left|h_{j d}\right|^{2}<\left|h_{i d}\right|^{2}\right) .
\end{aligned}
$$

709 Since the random variables $\left|h_{i e}\right|^{2}$ and $\left|h_{p e}\right|^{2}$ are independently 710 and exponentially distributed with respective means of $\sigma_{i e}^{2}$ and $711 \sigma_{p e}^{2}$, we readily arrive at

$$
\operatorname{Pr}\left(\left|h_{i e}\right|^{2}>\Lambda\left|h_{p e}\right|^{2} \gamma_{p}+\Lambda\right)=\frac{\sigma_{i e}^{2}}{\sigma_{p e}^{2} \gamma_{p} \Lambda+\sigma_{i e}^{2}} \exp \left(-\frac{\Lambda}{\sigma_{i e}^{2}}\right) .
$$

712 Substituting (B.5) and (B.8) into (B.7) gives (B.9), shown at the 713 bottom of the page, which is (50).

$717\left|h_{p d}\right|^{2}$, we can rewrite the terms $\operatorname{Pr}\left(\sum_{i \in \mathcal{D}_{n}}\left|h_{i d}\right|^{2}<\Lambda\right)$ and $718 \operatorname{Pr}\left(\sum_{i \in \mathcal{D}_{n}}\left|h_{i d}\right|^{2}<\gamma_{p} \Lambda\left|h_{p d}\right|^{2}+\Lambda\right)$ as $\operatorname{Pr}(X<\Lambda)$ and $\operatorname{Pr}(X<$ $719 \gamma_{p} \Lambda Y+\Lambda$ ), respectively. Noting that the fading coefficients of 720 all SR-SD channels, i.e. $\left|h_{i d}\right|^{2}$ for $i \in\{1,2, \cdots, N\}$, are assumed 721 to be i.i.d., we obtain the probability density function (PDF) of $722 X=\sum_{i \in \mathcal{D}_{n}}\left|h_{i d}\right|^{2}$ as

$$
f_{X}(x)=\frac{1}{\Gamma\left(\left|\mathcal{D}_{n}\right|\right) \sigma_{d}^{2\left|\mathcal{D}_{n}\right|}} x^{\left|\mathcal{D}_{n}\right|-1} \exp \left(-\frac{x}{\sigma_{d}^{2}}\right),
$$

723 where $\sigma_{d}^{2}=E\left(\left|h_{i d}\right|^{2}\right)$. Meanwhile, the random variable $Y=$ $724\left|h_{p d}\right|^{2}$ is exponentially distributed and its PDF is given by

$$
f_{Y}(y)=\frac{1}{\sigma_{p d}^{2}} \exp \left(-\frac{y}{\sigma_{p d}^{2}}\right)
$$

where $\sigma_{p d}^{2}=E\left(\left|h_{p d}\right|^{2}\right)$. Using (C.1), we arrive at

$$
\begin{aligned}
\operatorname{Pr}(X<\Lambda) & =\int_{0}^{\Lambda} \frac{1}{\Gamma\left(\left|\mathcal{D}_{n}\right|\right) \sigma_{d}^{2\left|\mathcal{D}_{n}\right|}} x^{\left|\mathcal{D}_{n}\right|-1} \exp \left(-\frac{x}{\sigma_{d}^{2}}\right) d x \\
& =\int_{0}^{\frac{\Lambda}{\sigma_{d}^{2}}} \frac{t^{\left|\mathcal{D}_{n}\right|-1}}{\Gamma\left(\left|\mathcal{D}_{n}\right|\right)} \exp (-t) d t \\
& =\Gamma\left(\frac{\Lambda}{\sigma_{d}^{2}},\left|\mathcal{D}_{n}\right|\right)
\end{aligned}
$$

where the second equality is obtained by substituting $\frac{x}{\sigma_{d}^{2}}=t$ and 726 $\Gamma(a, k)=\int_{0}^{a} \frac{t^{k-1}}{\Gamma(k)} \exp (-t) d t$ is known as the incomplete Gamma 727 function. Additionally, considering that the random variables $X 728$ and $Y$ are independent of each other, we obtain $\operatorname{Pr}\left(X<\gamma_{p} \Lambda Y+729\right.$ $\Lambda)$ as

$\operatorname{Pr}\left(X<\gamma_{p} \Lambda Y+\Lambda\right)=\int_{0}^{\Lambda} f_{X}(x) d x$

$$
+\int_{\Lambda}^{\infty} \int_{\frac{x}{-\gamma_{p} \Lambda}-\frac{1}{\gamma_{p}}}^{\infty} f_{X}(x) f_{Y}(y) d x d y .
$$

Substituting $f_{X}(x)$ and $f_{Y}(y)$ from (C.1) and (C.2) into (C.4) 731 yields

$\operatorname{Pr}\left(X<\gamma_{p} \Lambda Y+\Lambda\right)$

$$
\begin{aligned}
= & \Gamma\left(\frac{\Lambda}{\sigma_{d}^{2}},\left|\mathcal{D}_{n}\right|\right) \\
& +\int_{\Lambda}^{\infty} \frac{e^{1 /\left(\sigma_{p d}^{2} \gamma_{p}\right)} x^{\left|\mathcal{D}_{n}\right|-1}}{\Gamma\left(\left|\mathcal{D}_{n}\right|\right) \sigma_{d}^{2\left|\mathcal{D}_{n}\right|}} \exp \left(-\frac{x}{\sigma_{d}^{2}}-\frac{x}{\sigma_{p d}^{2} \gamma_{p} \Lambda}\right) d x \\
= & \Gamma\left(\frac{\Lambda}{\sigma_{d}^{2}},\left|\mathcal{D}_{n}\right|\right)+\frac{\left[1-\Gamma\left(\Lambda \sigma_{d}^{-2}+\sigma_{p d}^{-2} \gamma_{p}^{-1},\left|\mathcal{D}_{n}\right|\right)\right]}{\left(1+\sigma_{d}^{2} \sigma_{p d}^{-2} \gamma_{p}^{-1} \Lambda^{-1}\right)^{\left|\mathcal{D}_{n}\right|}} e^{1 /\left(\sigma_{p d}^{2} \gamma_{p}\right)},
\end{aligned}
$$

where the second equality is obtained by using $\frac{x}{\sigma_{d}^{2}}+\frac{x}{\sigma_{p d}^{2} \gamma_{p} \Lambda}=t .733$ Hence, we have completed the proof of (53) and (54) as (C.3) 734 and (C.5), respectively.

$$
\operatorname{Pr}\left(\left|h_{b e}\right|^{2}>\Lambda\right)=\sum_{i \in \mathcal{D}_{n}} \exp \left(-\frac{\Lambda}{\sigma_{i e}^{2}}\right)\left[1+\sum_{m=1}^{\left|\mathscr{D}_{n}\right|-1}(-1)^{\left|\mathcal{C}_{n}(m)\right|}\left(1+\sum_{j \in C_{n}(m)} \frac{\sigma_{i d}^{2}}{\sigma_{j d}^{2}}\right)^{-1}\right]
$$

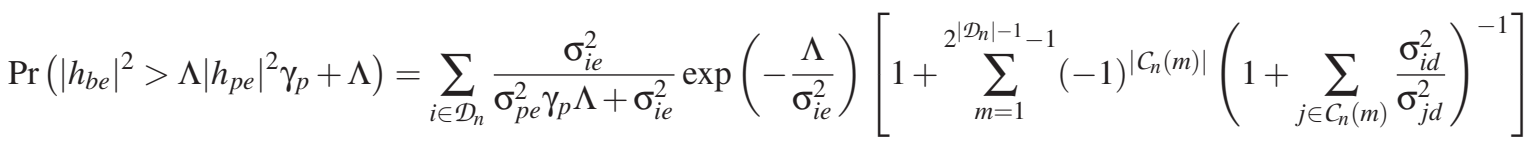




\section{REFERENCES}

1] J. Mitola and G. Q. Maguire, "Cognitive radio: Making software radios more personal," IEEE Pers. Commun., vol. 6, no. 4, pp. 13-18, Aug. 1999. 2] IEEE 802.22 Working Group, IEEE P802.22/D1.0 draft standard for wireless regional area networks part 22: Cognitive wireless RAN medium access control (MAC) and physical layer (PHY) specifications: Policies and procedures for operation in the TV bands, Apr. 2008.

3] G. Baldini, T. Sturman, A. R. Biswas, and R. Leschhorn, "Security aspects in software defined radio and cognitive radio networks: A survey and a way ahead," IEEE Commun. Surveys Tuts., vol. 14, no. 2, pp. 355-379, May 2012.

[4] D. Cabric, S. M. Mishra, and R. W. Brodersen, "Implementation issues in spectrum sensing for cognitive radios," in Proc. 38th Asil. Conf. Signal, Syst. Comput., Pacific Grove, CA, USA, Nov. 2004, pp. 772-776.

5] $\mathrm{H}$. $\mathrm{Li}$, "Cooperative spectrum sensing via belief propagation in spectrumheterogeneous cognitive radio systems," in Proc. IEEE WCNC, Sydney, N.S.W., Australia, Apr. 2010, pp. 1-6.

[6] J. Ma, G. Zhao, and Y. Li, "Soft combination and detection for cooperative spectrum sensing in cognitive radio networks," IEEE Trans. Wireless Commun., vol. 7, no. 11, pp. 4502-4507, Nov. 2008.

7] A. Ghasemi and E. S. Sousa, "Fundamental limits of spectrum-sharing in fading environments," IEEE Trans Wireless Commun., vol. 6, no. 2, pp. 649-658, Feb. 2007.

[8] R. Southwell, J. Huang, and X. Liu, "Spectrum mobility games," in Proc. 31st INFOCOM, Orlando, FL, USA, Mar. 2012, pp. 37-45.

9] I. F. Akyildiz, W.-Y. Lee, M. C. Vuran, and S. Mohanty, "A survey on spectrum management in cognitive radio networks," IEEE Commun. Mag., vol. 46, no. 4, pp. 40-48, Apr. 2008.

] H. Li and Z. Han, "Dogfight in spectrum: Combating primary user emulation attacks in cognitive radio systems part I: Known channel statistics," IEEE Trans. Wireless Commun., vol. 9, no. 11, pp. 3566-3577, Nov. 2010.

11] T. Brown and A. Sethi, "Potential cognitive radio denial-of-service vulnerabilities and protection countermeasures: A multi-dimensional analysis and assessment," in Proc. 2nd Int. Conf. CROWNCOM, Orlando, FL, USA, Aug. 2007, pp. 456-464.

2] S. Lakshmanan, C. Tsao, R. Sivakumar, and K. Sundaresan, "Securing wireless data networks against eavesdropping using smart antennas," in Proc. 28th ICDCS, Beijing, China, Jun. 2008, pp. 19-27.

13] A. Olteanu and Y. Xiao, "Security overhead and performance for aggregation with fragment retransmission (AFR) in very high-speed wireless 802.11 LANs," IEEE Trans. Wireless Commun., vol. 9, no. 1, pp. 218226, Jan. 2010.

14] Y. Xiao, V. K. Rayi, X. Du, F. Hu, and M. Galloway, "A survey of key management schemes in wireless sensor networks," Comput. Commun., vol. 30, no. 11-12, pp. 2314-2341, Sep. 2007.

15] A. Mukherjee, S. A. Fakoorian, J. Huang, and A. L. Swindlehurst, "Principles of physical layer security in multiuser wireless networks: A survey," IEEE Commun. Surveys Tuts., vol. 16, no. 3, pp. 1550-1573, Aug. 2014.

6] A. D. Wyner, "The wire-tap channel," Bell Syst. Tech. J., vol. 54, no. 8, pp. $1355-1387,1975$.

7] S. K. Leung-Yan-Cheong and M. E. Hellman, "The Gaussian wiretap channel," IEEE Trans. Inf. Theory, vol. 24, no. 4, pp. 451-456, Jul. 1978.

18] P. Parada and R. Blahut, "Secrecy capacity of SIMO and slow fading channels," in Proc. IEEE ISIT, Adelaide, SA, Australia, Sep. 2005, pp. 2152-2155.

9] M. Bloch, J. O. Barros, M. R. D. Rodrigues, and S. W. McLaughlin, "Wireless information-theoretic security," IEEE Trans. Inf. Theory, vol. 54, no. 6, pp. 2515-2534, Jun. 2008.

20] P. K. Gopala, L. Lai, and H. Gamal, "On the secrecy capacity of fading channels," IEEE Trans. Inf. Theory, vol. 54, no. 10, pp. 4687-4698, Oct. 2008.

21] Z. Li, W. Trappe, and R. Yates, "Secret communication via multi-antenna transmission," in Proc. 41st Conf. Inf. Sci. Syst., Baltimore, MD, USA, Mar. 2007, pp. 905-910.

22] F. Oggier and B. Hassibi, "The secrecy capacity of the MIMO wiretap channel," IEEE Trans. Inf. Theory, vol. 57, no. 8, pp. 4961-4972, Aug. 2007.

23] M. Yuksel and E. Erkip, "Secure communication with a relay helping the wiretapper," in Proc. IEEE Inf. Theory Workshop, Lake Tahoe, CA, USA, Sep. 2007, pp. 595-600.

4] L. Dong, Z. Han, A. P. Petropulu, and H. V. Poor, "Improving wireless physical layer security via cooperating relays," IEEE Trans. Signal Process., vol. 58, no. 3, pp. 1875-1888, Mar. 2010.

[25] Y. Zou, X. Wang, and W. Shen, "Optimal relay selection for physical-layer 812 security in cooperative wireless networks," IEEE J. Sel. Areas Commun., 813 vol. 31, no. 10, pp. 2099-2111, Oct. 2013.

[26] A. Mukherjee and A. Swindlehurst, "Robust beamforming for security 815 in MIMO wiretap channels with imperfect CSI," IEEE Trans. Signal 816 Process., vol. 59, no. 1, pp. 351-361, Jan. 2011.

[27] C. Jeong, I. Kim, and K. Dong, "Joint secure beamforming design at 818 the source and the relay for an amplify-and-forward MIMO untrusted 819 relay system," IEEE Trans. Signal Process., vol. 60, no. 1, pp. 310-325, 820 Jan. 2012.

[28] Y. Pei, Y.-C. Liang, K. C. Teh, and K. Li, "Secure communication in 822 multiantenna cognitive radio networks with imperfect channel state in- 823 formation," IEEE Trans. Signal Process., vol. 59, no. 4, pp. 1683-1693, 824 Apr. 2011.

[29] Y. Zou, X. Wang, and W. Shen, "Physical-layer security with multiuser 826 scheduling in cognitive radio networks," IEEE Trans. Commun., vol. 61, 827 no. 12 , pp. 5103-5113, Dec. 2013.

[30] Z. Shu, Y. Qian, and S. Ci, "On physical layer security for cognitive radio 829 networks," IEEE Netw. Mag., vol. 27, no. 3, pp. 28-33, Jun. 2013.

[31] Y. Zou, X. Wang, W. Shen, and L. Hanzo, "Security versus reliability 831 analysis of opportunistic relaying," IEEE Trans. Veh. Tech., vol. 63, no. 6, 832 pp. 2653-2661, Jun. 2014.

[32] L. Di Stefano and S. Mattoccia, "A sufficient condition based on the 834 Cauchy-Schwarz inequality for efficient template matching," in Proc. Int. 835 Conf. Image Process., Catalonia, Spain, Sep. 2003, pp. 269-272. 836

[33] M. Abramowitz and I. A. Stegun, Handbook of Mathematical Functions 837 with Formulas, Graphs, Mathematical Tables, 9th ed. New York, NY, 838 USA: Dover, 1970.

[34] Y. Zou, Y.-D. Yao, and B. Zheng, "Diversity-multiplexing tradeoff in 840 selective cooperation for cognitive radio," IEEE Trans. Commun., vol. 60, 841 no. 9, pp. 2467-2481, Sep. 2012.

842

[35] S. Goel and R. Negi, "Guaranteeing secrecy using artificial noise," IEEE 843 Trans. Wireless Commun., vol. 7, no. 6, pp. 2180-2189, Jun. 2008.

[36] W. Li, M. Ghogho, B. Chen, and C. Xiong, "Artificial noise by the 845 receiver: Outage secrecy capacity/region analysis," IEEE Commun. Lett., 846 vol. 16, no. 10, pp. 1628-1631, Oct. 2012.

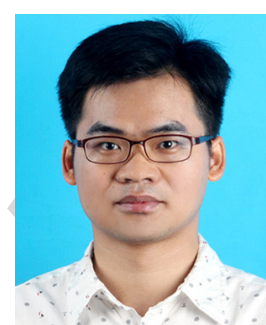

Yulong Zou (S'07-M'12-SM'13) received the 848 B.Eng. degree in information engineering from 849 NUPT, Nanjing, China, in July 2006, the first Ph.D. 850 degree in electrical engineering from the Stevens In- 851 stitute of Technology, New Jersey, the United States, 852 in May 2012, and the second Ph.D. degree in signal 853 and information processing from NUPT, Nanjing, 854 China, in July 2012. He is a Full Professor at the 855 Nanjing University of Posts and Telecommunica- 856 tions (NUPT), Nanjing, China. His research interests 857 span a wide range of topics in wireless commu- 858 nications and signal processing, including the cooperative communications, 859 cognitive radio, wireless security, and energy-efficient communications. $\quad 860$

He is currently serving as an editor for the IEEE Communications Surveys 861 \& Tutorials, IEEE COMmUniCATIONS LETTERs, EURASIP Journal on Ad- 862 vances in Signal Processing, and KSII Transactions on Internet and Information 863 Systems. He served as the lead guest editor for a special issue on "Security 864 Challenges and Issues in Cognitive Radio Networks" in the EURASIP Journal 865 on Advances in Signal Processing. He is also serving as the lead guest 866 editor for a special issue on "Security and Reliability Challenges in Industrial 867 Wireless Sensor Networks" in the IEEE TRANSACTIONS ON INDUSTRIAL 868 INFORMATICS. In addition, he has acted as symposium chairs, session chairs, 869 and TPC members for a number of IEEE sponsored conferences, including the 870 IEEE WIRELESS COMMUNICATIONS and Networking Conference (WCNC), 871 IEEE Global Communications Conference (GLOBECOM), IEEE International 872 Conference on Communications (ICC), IEEE Vehicular Technology Confer- 873 ence (VTC), International Conference on Communications in China (ICCC), 874 and so on. 


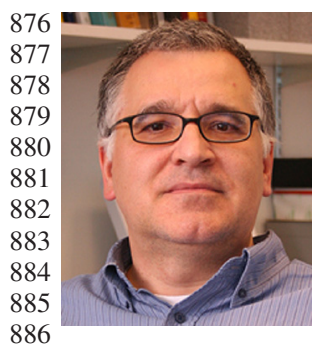

Benoit Champagne (S'87-M'89-SM'03) was born in Joliette (PQ), Canada, in 1961. He received the B.Ing. degree in engineering physics and the M.Sc. degree in physics from the University of Montreal in 1983 and 1985, respectively, and the Ph.D. degree in electrical engineering from the University of Toronto in 1990. From 1990 to 1999 , he was with INRS, University of Quebec, where he held the positions of Assistant and then Associate Professor. In 1999, he joined McGill University, Montreal, as an Associate Professor with the Department of Electrical and 887 Computer Engineering. He served as Associate Chairman of Graduate Studies 888 in the Department from 2004 to 2007 and is now a Full Professor.

889 His research interests focus on the investigation of new computational 890 algorithms for the digital processing of information bearing signals and over891 lap many sub-areas of statistical signal processing, including: detection and 892 estimation, sensor array processing, adaptive filtering, multirate systems, and 893 applications thereof to broadband voice and data communications. Over the 894 years, he has supervised many graduate students in these areas and co-authored 895 several papers, including key works on subspace tracking, speech enhancement, 896 time delay estimation and spread sources localization.

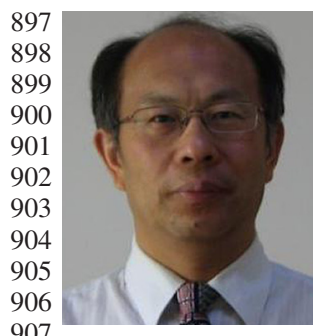

Wei-Ping Zhu (SM'97) received the B.E. and M.E. degrees from Nanjing University of Posts and Telecommunications, and the Ph.D. degree from Southeast University, Nanjing, China, in 1982, 1985, and 1991, respectively, all in electrical engineering. He was a Postdoctoral Fellow from 1991 to 1992 and a Research Associate from 1996 to 1998 with the Department of Electrical and Computer Engineering, Concordia University, Montreal, Canada. During 1993-1996, he was an Associate Professor with the Department of Information Engineering, 908 Nanjing University of Posts and Telecommunications. From 1998 to 2001, he 909 worked with hi-tech companies in Ottawa, Canada, including Nortel Networks 910 and SR Telecom Inc. Since July 2001, he has been with Concordia's Electrical 911 and Computer Engineering Department as a full-time faculty member, where 912 he is presently a Full Professor. His research interests include digital signal 913 processing fundamentals, speech and audio processing, and signal processing 914 for wireless communication with a particular focus on MIMO systems and 915 cooperative relay networks.

916 Dr. Zhu was an Associate Editor of the IEEE TRANSACTIONS ON CIRCUITS 917 And Systems PART I: Fundamental Theory and Applications from 2001 918 to 2003, and an Associate Editor of Circuits, Systems and Signal Processing 919 from 2006 to 2009. He was also a Guest Editor for the IEEE JOURNAL ON 920 SELECTEd AREAS IN COMMUNiCATIONS for the special issues of: Broadband 921 Wireless Communications for High Speed Vehicles, and Virtual MIMO during 922 2011-2013. Since 2011, he has served as an Associate Editor for the IEEE 923 Transactions on Circuits and Systems Part II: Express Briefs. Dr. 924 Zhu was the Secretary of Digital Signal Processing Technical Committee 925 (DSPTC) of the IEEE Circuits and System Society during 2012-2014, where 926 he is presently the Chair of the DSPTC.

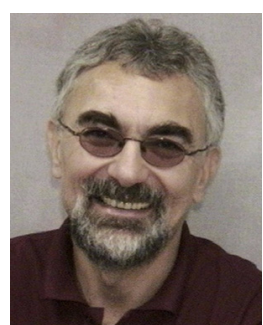

Lajos Hanzo received the degree in electronics in 927 1976 and the doctorate in 1983. In 2009 he was 928 awarded "Doctor Honoris Causa" by the Technical 929 University of Budapest. During his 37-year career in 930 telecommunications he has held various research and 931 academic posts in Hungary, Germany, and the UK. 932 Since 1986 he has been with the School of Electron- 933 ics and Computer Science, University of Southamp- 934 ton, UK, where he holds the chair in telecommunica- 935 tions. He has successfully supervised 80+ Ph.D. stu- 936 dents, co-authored 20 John Wiley/IEEE Press books 937 on mobile radio communications totalling in excess of 10000 pages, published 938 1400+ research entries at IEEE Xplore, acted both as TPC and General Chair of 939 IEEE conferences, presented keynote lectures and has been awarded a number 940 of distinctions. Currently he is directing a 100-strong academic research team, 941 working on a range of research projects in the field of wireless multimedia 942 communications sponsored by industry, the Engineering and Physical Sciences 943 Research Council (EPSRC) UK, the European Research Council's Advanced 944 Fellow Grant and the Royal Society's Wolfson Research Merit Award. He is an 945 enthusiastic supporter of industrial and academic liaison and he offers a range of 946 industrial courses. He is also a Governor of the IEEE VTS. During 2008-2012 947 he was the Editor-in-Chief of the IEEE Press and a Chaired Professor also 948 at Tsinghua University, Beijing. His research is funded by the European 949 Research Council's Senior Research Fellow Grant. For further information on 950 research in progress and associated publications please refer to http://www- 951 mobile.ecs.soton.ac.uk Lajos has $20000+$ citations. 
AUTHOR QUERY

NO QUERY. 


\section{Relay-Selection Improves the Security-Reliability Trade-Off in Cognitive Radio Systems}

Yulong Zou, Senior Member, IEEE, Benoit Champagne, Senior Member, IEEE, Wei-Ping Zhu, Senior Member, IEEE, and Lajos Hanzo, Fellow, IEEE

5 Abstract - We consider a cognitive radio (CR) network consisting 6 of a secondary transmitter (ST), a secondary destination (SD) and 7 multiple secondary relays (SRs) in the presence of an eavesdropper, 8 where the ST transmits to the SD with the assistance of SRs, while 9 the eavesdropper attempts to intercept the secondary transmission. 10 We rely on careful relay selection for protecting the ST-SD trans11 mission against the eavesdropper with the aid of both single-relay 12 and multi-relay selection. To be specific, only the "best" SR is cho13 sen in the single-relay selection for assisting the secondary trans14 mission, whereas the multi-relay selection invokes multiple SRs for 15 simultaneously forwarding the ST's transmission to the SD. We 16 analyze both the intercept probability and outage probability of 17 the proposed single-relay and multi-relay selection schemes for the 18 secondary transmission relying on realistic spectrum sensing. We 19 also evaluate the performance of classic direct transmission and 20 artificial noise based methods for the purpose of comparison with 21 the proposed relay selection schemes. It is shown that as the inter22 cept probability requirement is relaxed, the outage performance of 23 the direct transmission, the artificial noise based and the relay se-

24 lection schemes improves, and vice versa. This implies a trade-off 25 between the security and reliability of the secondary transmission 26 in the presence of eavesdropping attacks, which is referred to as 27 the security-reliability trade-off (SRT). Furthermore, we demon28 strate that the SRTs of the single-relay and multi-relay selection 29 schemes are generally better than that of classic direct trans30 mission, explicitly demonstrating the advantage of the proposed 31 relay selection in terms of protecting the secondary transmissions 32 against eavesdropping attacks. Moreover, as the number of SRs 33 increases, the SRTs of the proposed single-relay and multi-relay

Manuscript received May 7, 2014; revised August 21, 2014 and October 16, 2014; accepted November 27, 2014. This work was partially supported by the Priority Academic Program Development of Jiangsu Higher Education Institutions, the National Natural Science Foundation of China (Grant Nos. 61302104 and 61401223), the Scientific Research Foundation of Nanjing University of Posts and Telecommunications (Grant Nos. NY213014 and NY214001), the 1311 Talent Program of Nanjing University of Posts and Telecommunications, the Natural Science Foundation of Jiangsu Province (Grant No. BK20140887), and the Programme de bourses d'excellence pour etudiants etrangers (PBEEE) of the Government of Quebec. The associate editor coordinating the review of this paper and approving it for publication was $\mathrm{H}$. $\mathrm{Li}$

Y. Zou is with the School of Telecommunications and Information Engineering, Nanjing University of Posts and Telecommunications, Nanjing 210003, China (e-mail: yulong.zou@njupt.edu.cn).

B. Champagne is with the Department of Electrical \& Computer Engineering, McGill University, Montreal, QC H3A 1Y1, Canada (e-mail: benoit. champagne@mcgill.ca).

W.-P. Zhu is with the Department of Electrical \& Computer Engineering, Concordia University, Montreal, QC H3G 1M8, Canada (e-mail: weiping@ece. concordia.ca).

L. Hanzo is with the Department of Electronics and Computer Science, University of Southampton, Southampton SO17 1BJ, U.K. (e-mail: 1h@ecs. soton.ac.uk).

Color versions of one or more of the figures in this paper are available online at http://ieeexplore.ieee.org.

Digital Object Identifier 10.1109/TCOMM.2014.2377239 selection approaches significantly improve. Finally, our numerical 34 results show that as expected, the multi-relay selection scheme 35 achieves a better SRT performance than the single-relay selection. 36

Index Terms-Security-reliability trade-off, relay selection, 37 intercept probability, outage probability, eavesdropping attack, 38 cognitive radio.

\section{INTRODUCTION}

$\mathbf{T}$ HE security aspects of cognitive radio (CR) systems [1]- 41 [3] have attracted increasing attention from the research 42 community. Indeed, due to the highly dynamic nature of the CR 43 network architecture, legitimate CR devices become exposed 44 to both internal as well as to external attackers and hence they 45 are extremely vulnerable to malicious behavior. For example, 46 an illegitimate user may intentionally impose interference (i.e. 47 jamming) for the sake of artificially contaminating the CR envi- 48 ronment [4]. Hence, the CR users fail to accurately characterize 49 their surrounding radio environment and may become misled 50 or compromised, which leads to a malfunction. Alternatively, 51 an illegitimate user may attempt to tap the communications of 52 authorized CR users by eavesdropping, to intercept confidential 53 information.

Clearly, CR networks face diverse security threats during 55 both spectrum sensing [5], [6] as well as spectrum sharing [7], 56 spectrum mobility [8] and spectrum management [9]. Extensive 57 studies have been carried out for protecting CR networks both 58 against primary user emulation (PUE) [10] and against denial- 59 of-service (DoS) attacks [11]. In addition to PUE and DoS at- 60 tacks, eavesdropping is another main concern in protecting the 61 data confidentiality [12], although it has received less attention 62 in the literature on CR network security. Traditionally, crypto- 63 graphic techniques are employed for guaranteeing transmission 64 confidentiality against an eavesdropping attack. However, this 65 introduces a significant computational overhead [13] as well as 66 imposing additional system complexity in terms of the secret 67 key management [14]. Furthermore, the existing cryptographic 68 approaches are not perfectly secure and can still be decrypted 69 by an eavesdropper (E), provided that it has the capacity to carry 70 out exhaustive key search with the aid of brute-force attack [15]. 71

Physical-layer security [16], [17] is emerging as an efficient 72 approach for defending authorized users against eavesdropping 73 attacks by exploiting the physical characteristics of wireless 74 channels. In [17], Leung-Yan-Cheong and Hellman demon- 75 strated that perfectly secure and reliable transmission can be 76 achieved, when the wiretap channel spanning from the source 77 to the eavesdropper is a further degraded version of the main 78 
79 channel between the source and destination. They also showed 80 that the maximal secrecy rate achieved at the legitimate desti81 nation, which is termed the secrecy capacity, is the difference 82 between the capacity of the main channel and that of the 83 wiretap channel. In [18]-[20], the secrecy capacity limits of 84 wireless fading channels were further developed and character85 ized from an information-theoretic perspective, demonstrating 86 the detrimental impact of wireless fading on the physical87 layer security. To combat the fading effects, both multiple-input 88 multiple-output (MIMO) schemes [21], [22] as well as coop89 erative relaying [23]-[25] and beamforming techniques [26], 90 [27] were investigated for the sake of enhancing the achievable 91 wireless secrecy capacity. Although extensive research efforts 92 were devoted to improving the security of traditional wireless 93 networks [16]-[27], less attention has been dedicated to CR 94 networks. In [28] and [29], the achievable secrecy rate of 95 the secondary transmission was investigated under a specific 96 quality-of-service (QoS) constraint imposed on the primary 97 transmission. Additionally, an overview of the physical-layer 98 security aspects of CR networks was provided in [30], where 99 several security attacks as well as the related countermeasures 100 are discussed. In contrast to conventional non-cognitive wire101 less networks, the physical-layer security of CR networks has to 102 consider diverse additional challenges, including the protection 103 of the primary user's QoS and the mitigation of the mutual 104 interference between the primary and secondary transmissions. 105 Motivated by the above considerations, we explore the 106 physical-layer security of a CR network comprised of a sec107 ondary transmitter (ST) communicating with a secondary des108 tination (SD) with the aid of multiple secondary relays (SRs) 109 in the presence of an unauthorized attacker. Our main focus 110 is on investigating the security-reliability trade-off (SRT) of 111 the cognitive relay transmission in the presence of realistic 112 spectrum sensing. The notion of the SRT in wireless physical113 layer security was introduced and examined in [31], where the 114 security and reliability was characterized in terms of the inter115 cept probability and outage probability, respectively. In contrast 116 to the conventional non-cognitive wireless networks studied in 117 [31], the SRT analysis of CR networks presented in this work 118 additionally takes into account the mutual interference between 119 the primary user (PU) and secondary user (SU).

120 The main contributions of this paper are summarized as 121 follows.

- We propose two relay selection schemes, namely both single-relay and multi-relay selection, for protecting the secondary transmissions against eavesdropping attacks. More specifically, in the single-relay selection (SRS) scheme, only a single relay is chosen from the set of multiple SRs for forwarding the secondary transmissions from the ST to the SD. By contrast, the multi-relay selection (MRS) scheme employs multiple SRs for simultaneously assisting the ST-SD transmissions.

- We present the mathematical SRT analysis of the proposed SRS and MRS schemes in the presence of realistic spectrum sensing. Closed-form expressions are derived for the intercept probability (IP) and outage probability (OP) of both schemes for transmission over Rayleigh fading channels. The numerical SRT results of conventional direct

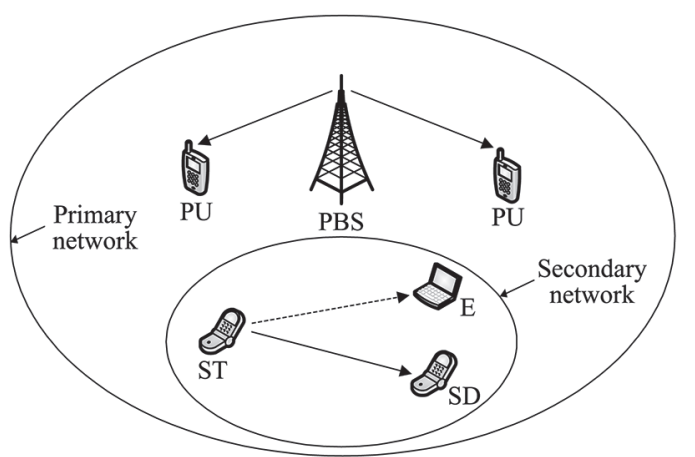

PBS: Primary Base Station PU: Primary User

ST: Secondary Transmitter SD: Secondary Destination

E: Eavesdropper

Fig. 1. A primary wireless network in coexistence with a secondary CR network.

transmission and artificial noise based schemes are also 137 provided for comparison purposes.

- It is shown that as the spectrum sensing reliability is 139 increased and/or the false alarm probability is reduced, the 140 SRTs of both the SRS and MRS schemes are improved. 141 Numerical results demonstrate that the proposed SRS and 142 MRS schemes generally outperform the conventional di- 143 rect transmission and artificial noise based approaches in 144 terms of their SRTs.

The remainder of this paper is organized as follows. 146 Section II presents the system model of physical-layer security 147 in CR networks in the context of both the direct transmission as 148 well as the SRS and MRS schemes. In Section III, we analyze 149 the SRTs of these schemes in the presence of realistic spectrum 150 sensing over Rayleigh fading channels. Next, numerical SRT 151 results of the direct transmission, SRS and MRS schemes are 152 given in Section IV, where the SRT performance of the artificial 153 noise based scheme is also numerically evaluated for com- 154 parison purposes. Finally, Section V provides our concluding 155 remarks.

II. Relay Selection Aided Protection Against 157 EAVESDROPPING IN CR NETWORKS

We first introduce the overall system model of physical-layer 159 security in CR networks. We then present the signal model of 160 the conventional direct transmission approach, which will serve 161 as our benchmarker, as well as of the SRS and MRS schemes 162 for improving the CR system's security against eavesdropping 163 attacks.

\section{A. System Model}

As shown in Fig. 1, we consider a primary network in 166 coexistence with a secondary network (also referred to as a $C R 167$ network). The primary network includes a primary base station 168 (PBS) and multiple primary users (PUs), which communicate 169 with the PBS over the licensed spectrum. By contrast, the 170 secondary network consisting of one or more STs and SDs 171 exploits the licensed spectrum in an opportunistic way. To 172 
173 be specific, a particular ST should first detect with the aid 174 of spectrum sensing whether or not the licensed spectrum is 175 occupied by the PBS. If so, the ST is not at liberty to transmit 176 to avoid interfering with the PUs. If alternatively, the licensed 177 spectrum is deemed to be unoccupied (i.e. a spectrum hole 178 is detected), then the ST may transmit to the SD over the 179 detected spectrum hole. Meanwhile, E attempts to intercept the 180 secondary transmission from the ST to the SD. For notational 181 convenience, let $H_{0}$ and $H_{1}$ represent the event that the licensed 182 spectrum is unoccupied and occupied by the PBS during a 183 particular time slot, respectively. Moreover, let $\hat{H}$ denote the 184 status of the licensed spectrum detected by spectrum sensing. 185 Specifically, $\hat{H}=H_{0}$ represents the case that the licensed 186 spectrum is deemed to be unoccupied, while $\hat{H}=H_{1}$ indicates 187 that the licensed spectrum is deemed to be occupied.

188 The probability $P_{d}$ of correct detection of the presence of $189 \mathrm{PBS}$ and the associated false alarm probability $P_{f}$ are defined 190 as $P_{d}=\operatorname{Pr}\left(\hat{H}=H_{1} \mid H_{1}\right)$ and $P_{f}=\operatorname{Pr}\left(\hat{H}=H_{1} \mid H_{0}\right)$, respectively. 191 Due to the background noise and fading effects, it is impossible 192 to achieve perfectly reliable spectrum sensing without missing 193 the detection of an active PU and without false alarm, which 194 suggests that a spectral band is occupied by a PU, when it 195 is actually unoccupied. Moreover, the missed detection of the 196 presence of PBS will result in interference between the PU 197 and SU. To guarantee that the interference imposed on the 198 PUs is below a tolerable level, both the successful detection 199 probability (SDP) $P_{d}$ and false alarm probability (FAP) $P_{f}$ 200 should be within a meaningful target range. For example, the 201 IEEE 802.22 standard requires $P_{d}>0.9$ and $P_{f}<0.1$ [2]. For 202 better protection of PUs, we consider $P_{d}=0.99$ and $P_{f}=0.01$, 203 unless otherwise stated. Additionally, we consider a Rayleigh 204 fading model for characterizing all the channels between any 205 two nodes of Fig. 1. Finally, all the received signals are assumed 206 to be corrupted by additive white Gaussian noise (AWGN) 207 having a zero mean and a variance of $N_{0}$.

\section{B. Direct Transmission}

209 Let us first consider the conventional direct transmission 210 as a benchmark scheme. Let $x_{p}$ and $x_{s}$ denote the random 211 symbols transmitted by the PBS and the ST at a particular 212 time instance. Without loss of generality, we assume $E\left[\left|x_{p}\right|^{2}\right]=$ $213 E\left[\left|x_{S}\right|^{2}\right]=1$, where $E[\cdot]$ represents the expected value operator. 214 The transmit powers of the PBS and ST are denoted by $P_{p}$ and $215 P_{S}$, respectively. Given that the licensed spectrum is deemed to 216 be unoccupied by the PBS (i.e. $\hat{H}=H_{0}$ ), ST transmits its signal $217 x_{S}$ at a power of $P_{S}$. Then, the signal received at the SD can be 218 written as

$$
y_{d}=h_{s d} \sqrt{P_{s}} x_{s}+h_{p d} \sqrt{\alpha P_{p}} x_{p}+n_{d}
$$

219 where $h_{s d}$ and $h_{p d}$ represent the fading coefficients of the 220 channel spanning from ST to SD and that from PBS to SD, 221 respectively. Furthermore, $n_{d}$ represents the AWGN received at $222 \mathrm{SD}$ and the random variable (RV) $\alpha$ is defined as

$$
\alpha= \begin{cases}0, & H_{0} \\ 1, & H_{1},\end{cases}
$$

where $H_{0}$ represents that the licensed spectrum is unoccupied 223 by PBS and no primary signal is transmitted, leading to $\alpha=0.224$ By contrast, $H_{1}$ represents that PBS is transmitting its signal $x_{p} 225$ over the licensed spectrum, thus $\alpha=1$. Meanwhile, due to the 226 broadcast nature of the wireless medium, the ST's signal will 227 be overheard by E and the overheard signal can be expressed as 228

$$
y_{e}=h_{s e} \sqrt{P_{s}} x_{s}+h_{p e} \sqrt{\alpha P_{p}} x_{p}+n_{e},
$$

where $h_{s e}$ and $h_{p e}$ represent the fading coefficients of the 229 channel spanning from ST to $\mathrm{E}$ and that from PBS to E, 230 respectively, while $n_{e}$ represents the AWGN received at E. 231 Upon combining Shannon's capacity formula [31] with (1), we 232 obtain the capacity of the ST-SD channel as

$$
C_{s d}=\log _{2}\left(1+\frac{\left|h_{s d}\right|^{2} \gamma_{s}}{\alpha\left|h_{p d}\right|^{2} \gamma_{p}+1}\right)
$$

where $\gamma_{s}=P_{s} / N_{0}$ and $\gamma_{p}=P_{p} / N_{0}$. Similarly, the capacity of the 234 ST-E channel is obtained from (3) as

$$
C_{s e}=\log _{2}\left(1+\frac{\left|h_{s e}\right|^{2} \gamma_{s}}{\alpha\left|h_{p e}\right|^{2} \gamma_{p}+1}\right) \text {. }
$$

\section{Single-Relay Selection}

In this subsection, we consider the cognitive relay network 237 of Fig. 2, where both SD and E are assumed to be beyond 238 the coverage area of the ST [24], [25], and $N$ secondary 239 relays (SRs) are employed for assisting the cognitive ST-SD 240 transmission. We assume that a common control channel (CCC) 241 [6] is available for coordinating the actions of the different 242 network nodes and the decode-and-forward (DF) relaying using 243 two adjacent time slots is employed. More specifically, once 244 the licensed spectrum is deemed to be unoccupied, the ST first 245 broadcasts its signal $x_{s}$ to the $N$ SRs, which attempt to decode 246 $x_{s}$ from their received signals. For notational convenience, let 247 $\mathcal{D}$ represent the set of SRs that succeed in decoding $x_{s}$. Given 248 $N$ SRs, there are $2^{N}$ possible subsets $\mathcal{D}$, thus the sample space 249 of $\mathcal{D}$ is formulated as

$$
\Omega=\left\{\emptyset, \mathcal{D}_{1}, \mathcal{D}_{2}, \cdots, \mathcal{D}_{n}, \cdots, \mathcal{D}_{2^{N-1}}\right\}
$$

where $\emptyset$ represents the empty set and $\mathcal{D}_{n}$ represents the $n$-th 251 non-empty subset of the $N$ SRs. If the set $\mathcal{D}$ is empty, implying 252 that no SR decodes $x_{s}$ successfully, then all the SRs remain 253 silent and thus both SD and E are unable to decode $x_{s}$ in this 254 case. If the set $\mathcal{D}$ is non-empty, a specific $\mathrm{SR}$ is chosen from 255 $\mathcal{D}$ to forward its decoded signal $x_{s}$ to SD. Therefore, given 256 $\hat{H}=H_{0}$ (i.e. the licensed spectrum is deemed unoccupied), ST 257 broadcasts its signal $x_{S}$ to $N$ SRs at a power of $P_{S}$ and a rate of 258 $R$. Hence, the signal received at a specific $\mathrm{SR}_{i}$ is given by

$$
y_{i}=h_{s i} \sqrt{P_{s}} x_{s}+h_{p i} \sqrt{\alpha P_{p}} x_{p}+n_{i},
$$

where $h_{s i}$ and $h_{p i}$ represent the fading coefficients of the ST-SR 260 channel and that of the $\mathrm{PBS}_{-} \mathrm{SR}_{i}$ channel, respectively, with 261 
$262 n_{i}$ representing the $\mathrm{AWGN}$ at $\mathrm{SR}_{i}$. From (7), we obtain the 263 capacity of the $\mathrm{ST}_{-} \mathrm{SR}_{i}$ channel as

$$
C_{s i}=\frac{1}{2} \log _{2}\left(1+\frac{\left|h_{s i}\right|^{2} \gamma_{s}}{\alpha\left|h_{p i}\right|^{2} \gamma_{p}+1}\right),
$$

264 where the factor $\frac{1}{2}$ arises from the fact that two orthogonal time 265 slots are required for completing the message transmission from $266 \mathrm{ST}$ to $\mathrm{SD}$ via $\mathrm{SR}_{i}$. According to Shannon's coding theorem, 267 if the data rate is higher than the channel capacity, the re268 ceiver becomes unable to successfully decode the source signal, 269 regardless of the decoding algorithm adopted. Otherwise, the 270 receiver can succeed in decoding the source signal. Thus, using 271 (8), we can describe the event of $\mathcal{D}=\emptyset$ as

$$
C_{s i}<R, \quad i \in\{1,2, \cdots, N\}
$$

272 Meanwhile, the event of $\mathcal{D}=\mathcal{D}_{n}$ is described as

$$
\begin{aligned}
& C_{s i}>R, \quad i \in \mathcal{D}_{n} \\
& C_{s j}<R, \quad j \in \overline{\mathcal{D}}_{n},
\end{aligned}
$$

273 where $\overline{\mathcal{D}}_{n}$ represents the complementary set of $\mathcal{D}_{n}$. Without 274 loss of generality, we assume that $\mathrm{SR}_{i}$ is chosen within $\mathcal{D}_{n}$ to 275 transmit its decoded result $x_{s}$ at a power of $P_{s}$, thus the signal 276 received at SD can be written as

$$
y_{d}=h_{i d} \sqrt{P_{s}} x_{s}+h_{p d} \sqrt{\alpha P_{p}} x_{p}+n_{d}
$$

277 where $h_{i d}$ represents the fading coefficient of the $\mathrm{SR}_{i}-\mathrm{SD}$ 278 channel. From (11), the capacity of the $\mathrm{SR}_{i}-\mathrm{SD}$ channel is 279 given by

$$
C_{i d}=\frac{1}{2} \log _{2}\left(1+\frac{\left|h_{i d}\right|^{2} \gamma_{s}}{\alpha\left|h_{p d}\right|^{2} \gamma_{p}+1}\right),
$$

280 where $i \in \mathcal{D}_{n}$. In general, the specific $\mathrm{SR}_{i}$ having the highest 281 instantaneous capacity to SD is chosen as the "best" SR for as282 sisting the ST's transmission. Therefore, the best relay selection 283 criterion is expressed from (12) as

$$
\text { Best SR }=\arg \max _{i \in \mathcal{D}_{n}} C_{i d}=\arg \max _{i \in \mathcal{D}_{n}}\left|h_{i d}\right|^{2}
$$

284 which shows that only the channel state information (CSI) $\left|h_{i d}\right|^{2}$ 285 is required for performing the relay selection without the need 286 for the eavesdropper's CSI knowledge. Upon combining (12) 287 and (13), we obtain the capacity of the channel spanning from 288 the "best" SR to SD as

$$
C_{b d}=\frac{1}{2} \log _{2}\left(1+\frac{\gamma_{s}}{\alpha\left|h_{p d}\right|^{2} \gamma_{p}+1} \max _{i \in \mathcal{D}_{n}}\left|h_{i d}\right|^{2}\right),
$$

289 where the subscript ' $b$ ' in $C_{b d}$ denotes the best SR. It is observed 290 from (14) that the legitimate transmission capacity of the SRS 291 scheme is determined by the maximum of independent random 292 variables (RVs) $\left|h_{i d}\right|^{2}$ for different SRs. By contrast, one can 293 see from (4) that the capacity of classic direct transmission is 294 affected by the single RV $\left|h_{s d}\right|^{2}$. If all RVs $\left|h_{i d}\right|^{2}$ and $\left|h_{s d}\right|^{2}$ are 295 independent and identically distributed (i.i.d), it would be most 296 likely that $\max _{i \in \mathcal{D}_{n}}\left|h_{i d}\right|^{2}$ is much higher than $\left|h_{s d}\right|^{2}$ for a sufficiently

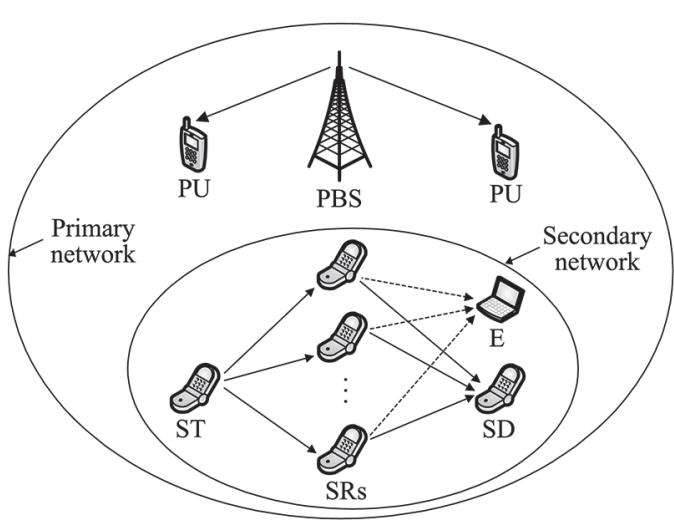

SRs: Secondary Relays

Fig. 2. A cognitive relay network consists of one ST, one SD and $N$ SRs in the presence of an E.

large number of SRs, resulting in a performance improvement 297 for the SRS scheme over the classic direct transmission. How- 298 ever, if the RVs $\left|h_{i d}\right|^{2}$ and $\left|h_{s d}\right|^{2}$ are non-identically distributed 299 and the mean value of $\left|h_{s d}\right|^{2}$ is much higher than that of $\left|h_{i d}\right|^{2}, 300$ then it may be more likely that $\max _{i \in \mathcal{D}_{n}}\left|h_{i d}\right|^{2}$ is smaller than $\left|h_{s d}\right|^{2} 301$ for a given number of SRs. In this extreme case, the classic 302 direct transmission may perform better than the SRS scheme. 303 It is worth mentioning that in practice, the average fading gain 304 of the $\mathrm{SR}_{i}-\mathrm{SD}$ channel, $\left|h_{i d}\right|^{2}$, should not be less than that 305 of the ST-SD channel $\left|h_{s d}\right|^{2}$, since SRs are typically placed 306 in the middle between the ST and SD. Hence, a performance 307 improvement for the SRS scheme over classic direct transmis- 308 sion would be achieved in practical wireless systems. Note 309 that although a factor $1 / 2$ in (14) is imposed on the capacity 310 of the main channel, it would not affect the performance of 311 the SRS scheme from a SRT perspective, since the capacity 312 of the wiretap channel is also multiplied by $1 / 2$ as will be 313 shown in (16).

314

Additionally, given that the selected SR transmits its 315 decoded result $x_{s}$ at a power of $P_{s}$, the signal received at $\mathrm{E}$ is 316 expressed as

$$
y_{e}=h_{b e} \sqrt{P_{s}} x_{s}+h_{p e} \sqrt{\alpha P_{p}} x_{p}+n_{e},
$$

where $h_{b e}$ and $h_{p e}$ represent the fading coefficients of the chan- 318 nel from "best" SR to E and that from PBS to E, respectively. 319 From (15), the capacity of the channel spanning from the "best" 320 $\mathrm{SR}$ to $\mathrm{E}$ is given by

$$
C_{b e}=\frac{1}{2} \log _{2}\left(1+\frac{\left|h_{b e}\right|^{2} \gamma_{s}}{\alpha\left|h_{p e}\right|^{2} \gamma_{p}+1}\right),
$$

where $b \in \mathcal{D}_{n}$ is determined by the relay selection criterion 322 given in (13). As shown in (16), the eavesdropper's channel 323 capacity is affected by the channel state information (CSI) 324 $\left|h_{b e}\right|^{2}$ of the wiretap channel spanning from the "best" relay to 325 the eavesdropper. However, one can see from (13) that the best 326 relay is selected from the decoding set $\mathcal{D}_{n}$ solely based on the 327 main channel's CSI $\left|h_{i d}\right|^{2}$ i.e. without taking into account the 328 eavesdropper's CSI knowledge of $\left|h_{i e}\right|^{2}$. This means that the 329 selection of the best relay aiming for maximizing the legitimate 330 transmission capacity of (14) would not lead to significantly 331 
332 beneficial or adverse impact on the eavesdropper's channel 333 capacity, since the main channel and the wiretap channel are 334 independent of each other.

335 For example, if the random variables (RVs) $\left|h_{i e}\right|^{2}$ related to 336 the different relays are i.i.d, we can readily infer by the law 337 of total probability that $\left|h_{b e}\right|^{2}$ has the same probability den338 sity function (PDF) as $\left|h_{i e}\right|^{2}$, implying that the eavesdropper's 339 channel capacity of (16) is not affected by the selection of the 340 best relay given by (13). Therefore, the SRS scheme has no 341 obvious advantage over the classic direct transmission in terms 342 of minimizing the capacity of the wiretap channel. To elaborate 343 a little further, according to the SRT trade-off, a reduction of 344 the outage probability (OP) due to the capacity enhancement 345 of the main channel achieved by using the selection of the 346 best relay would be converted into an intercept probability 347 (IP) improvement, which will be numerically illustrated in 348 Section IV.

\section{D. Multi-Relay Selection}

350 This subsection presents a MRS scheme, where multiple SRs 351 are employed for simultaneously forwarding the source signal $352 x_{s}$ to SD. To be specific, ST first transmits $x_{s}$ to $N$ SRs over a 353 detected spectrum hole. As mentioned in Subsection II-C, we 354 denote by $\mathcal{D}$ the set of SRs that successfully decode $x_{s}$. If $\mathcal{D}$ 355 is empty, all SRs fail to decode $x_{s}$ and will not forward the 356 source signal, thus both SD and E are unable to decode $x_{s}$. If $357 \mathcal{D}$ is non-empty (i.e. $\mathcal{D}=\mathcal{D}_{n}$ ), all SRs within $\mathcal{D}_{n}$ are utilized 358 for simultaneously transmitting $x_{s}$ to SD. This differs from the 359 SRS scheme, where only a single $\mathrm{SR}$ is chosen from $\mathcal{D}_{n}$ for 360 forwarding $x_{s}$ to SD. To make effective use of multiple SRs, a 361 weight vector denoted by $w=\left[w_{1}, w_{2}, \cdots, w_{\left|\mathcal{D}_{n}\right|}\right]^{T}$ is employed 362 at the SRs for transmitting $x_{s}$, where $\left|\mathcal{D}_{n}\right|$ is the cardinality of 363 the set $\mathcal{D}_{n}$. For the sake of a fair comparison with the SRS 364 scheme in terms of power consumption, the total transmit power 365 across all SRs within $\mathcal{D}_{n}$ shall be constrained to $P_{s}$ and thus the 366 weight vector $w$ should be normalized according to $\|w\|=1$. 367 Thus, given $\mathcal{D}=\mathcal{D}_{n}$ and considering that all SRs within $\mathcal{D}_{n}$ are 368 selected for simultaneously transmitting $x_{s}$ with a weight vector $369 w$, the signal received at SD is expressed as

$$
y_{d}^{\text {multi }}=\sqrt{P_{s}} w^{T} H_{d} x_{s}+\sqrt{\alpha P_{p}} h_{p d} x_{p}+n_{d}
$$

370 where $H_{d}=\left[h_{1 d}, h_{2 d}, \cdots, h_{\left|\mathcal{D}_{n}\right| d}\right]^{T}$. Similarly, the signal received 371 at E can be written as

$$
y_{e}^{\text {multi }}=\sqrt{P_{s}} w^{T} H_{e} x_{s}+\sqrt{\alpha P_{p}} h_{p e} x_{p}+n_{e},
$$

372 where $H_{e}=\left[h_{1 e}, h_{2 e}, \cdots, h_{\left|\mathcal{D}_{n}\right| e}\right]^{T}$. From (17) and (18), the 373 signal-to-interference-plus-noise ratios (SINRs) at SD and E 374 are, respectively, given by

$$
\operatorname{SINR}_{d}^{\text {multi }}=\frac{\gamma_{s}}{\alpha\left|h_{p d}\right|^{2} \gamma_{p}+1}\left|w^{T} H_{d}\right|^{2},
$$

375 and

$$
\operatorname{SINR}_{e}^{\text {multi }}=\frac{\gamma_{s}}{\alpha\left|h_{p e}\right|^{2} \gamma_{p}+1}\left|w^{T} H_{e}\right|^{2}
$$

In this work, the weight vector $w$ is optimized by maximizing 376 the SINR at SD, yielding

$$
\max _{w} \operatorname{SINR}_{d}^{\text {multi }}, \quad \text { s.t. }\|w\|=1
$$

where the constraint is used for normalization purposes. Using 378 the Cauchy-Schwarz inequality [32], we can readily obtain the 379 optimal weight vector $w_{\text {opt }}$ from (21) as

$$
w_{\mathrm{opt}}=\frac{H_{d}^{*}}{\left|H_{d}\right|},
$$

which indicates that the optimal vector design only requires the 381 SR-SD CSI $H_{d}$, whilst dispensing with the eavesdropper's CSI 382 $H_{e}$. Substituting the optimal vector $w_{\text {opt }}$ from (22) into (19) and 383 (20) and using Shannon's capacity formula, we can obtain the 384 channel capacities achieved at both SD and E as

$$
C_{d}^{\text {multi }}=\frac{1}{2} \log _{2}\left(1+\frac{\gamma_{s}}{\alpha \gamma_{p}\left|h_{p d}\right|^{2}+1} \sum_{i \in \mathcal{D}_{n}}\left|h_{i d}\right|^{2}\right),
$$

and

$$
C_{e}^{\text {multi }}=\frac{1}{2} \log _{2}\left(1+\frac{\gamma_{s}}{\alpha \gamma_{p}\left|h_{p e}\right|^{2}+1} \frac{\left|H_{d}^{H} H_{e}\right|^{2}}{\left|H_{d}\right|^{2}}\right),
$$

for $\mathcal{D}=\mathcal{D}_{n}$, where $H$ represents the Hermitian transpose. One 387 can observe from (14) and (23) that the difference between the 388 capacity expressions $C_{b d}$ and $C_{d}^{\text {multi }}$ only lies in the fact that 389 the maximum of RVs $\left|h_{i d}\right|^{2}$ for different SRs (i.e., $\max _{i \in \mathcal{D}_{n}}\left|h_{i d}\right|^{2}$ ) 390 is used for the SRS scheme, while the sum of RVs $\left|h_{i d}\right|^{2} 391$ (i.e., $\sum_{i \in \mathcal{D}_{n}}\left|h_{i d}\right|^{2}$ ) is employed for the MRS scheme. Clearly, 392 we have $\sum_{i \in \mathcal{D}_{n}}\left|h_{i d}\right|^{2}>\max _{i \in \mathcal{D}_{n}}\left|h_{i d}\right|^{2}$, resulting in a performance 393 gain for MRS over SRS in terms of maximizing the legitimate 394 transmission capacity. Moreover, since the main channel $H_{d} 395$ and the wiretap channel $H_{e}$ are independent of each other, the 396 optimal weights assigned for the multiple relays based on $H_{d} 397$ will only slightly affect the eavesdropper's channel capacity. 398 This means that the MRS and SRS schemes achieve more or 399 less the same performance in terms of the capacity of the wire- 400 tap channel. Nevertheless, given a fixed outage requirement, 401 the MRS scheme can achieve a better intercept performance 402 than the SRS scheme, because according to the SRT, an outage 403 reduction achieved by the capacity enhancement of the legiti- 404 mate transmission relying on the MRS would be converted into 405 an intercept improvement. To be specific, given an enhanced 406 capacity of the legitimate transmission, we may increase the 407 data rate $R$ based on the OP definition of (25) for maintaining 408 a fixed OP, which, in turn leads to a reduction of the IP, since a 409 higher data rate would result in a lower IP, according to the IP 410 definition of (26).

411

It needs to be pointed out that in the MRS scheme, a 412 high-complexity symbol-level synchronization is required for 413 multiple distributed SRs, when simultaneously transmitting to 414 SD, whereas the SRS does not require such a complex synchro- 415 nization process. Thus, the performance improvement of MRS 416 over SRS is achieved at the cost of a higher implementation 417 
418 complexity. Additionally, the synchronization imperfections of 419 the MRS scheme will impose a performance degradation, which 420 may even lead to a performance for the MRS scheme becoming 421 worse than that of the SRS scheme.

422 Throughout this paper, the Rayleigh model is used for char423 acterizing the fading amplitudes (e.g., $\left|h_{s d}\right|,\left|h_{s i}\right|,\left|h_{i d}\right|$, etc.) of 424 wireless channels, which, in turn, implies that the fading square 425 magnitudes $\left|h_{s d}\right|^{2},\left|h_{s i}\right|^{2}$ and $\left|h_{i d}\right|^{2}$ are exponentially distributed 426 random variables (RVs). So far, we have completed the presen427 tation of the signal model of the direct transmission, of the SRS, 428 and of the MRS schemes for CR networks applications in the 429 presence of eavesdropping attacks.

\section{III. SRT ANALYSIS OVER RAYLEIGH FADING CHANNELS}

431 This section presents the SRT analysis of the direct transmis432 sion, SRS and MRS schemes over Rayleigh fading channels. 433 As discussed in [31], the security and reliability are quantified 434 in terms of the IP and OP experienced by the eavesdropper and 435 destination, respectively. It is pointed out that in CR networks, 436 ST starts to transmit its signal only when an available spectrum 437 hole is detected. Similarly to [34], the OP and IP are thus 438 calculated under the condition that the licensed spectrum is 439 detected to be unoccupied by the PBS. The following gives the 440 definition of OP and IP.

441 Definition 1: Let $C_{d}$ and $C_{e}$ represent the channel capacities 442 achieved at the destination and eavesdropper, respectively. The $443 \mathrm{OP}$ and IP are, respectively, defined as

$$
P_{\text {out }}=\operatorname{Pr}\left(C_{d}<R \mid \hat{H}=H_{0}\right),
$$

444 and

$$
P_{\text {int }}=\operatorname{Pr}\left(C_{e}>R \mid \hat{H}=H_{0}\right),
$$

445 where $R$ is the data rate.

\section{A. Direct Transmission}

447 Let us first analyze the SRT performance of the conventional 448 direct transmission. Given that a spectrum hole has been de449 tected, the OP of direct transmission is obtained from (25) as

$$
P_{\text {out }}^{\text {direct }}=\operatorname{Pr}\left(C_{s d}<R \mid \hat{H}=H_{0}\right),
$$

450 where $C_{s d}$ is given by (4). Using the law of total probability, we 451 can rewrite $(27)$ as

$$
P_{\text {out }}^{\text {direct }}=\operatorname{Pr}\left(C_{s d}<R, H_{0} \mid \hat{H}=H_{0}\right)+\operatorname{Pr}\left(C_{s d}<R, H_{1} \mid \hat{H}=H_{0}\right),
$$

452 which can be further expressed as

$$
\begin{aligned}
P_{\text {out }}^{\text {direct }}=\operatorname{Pr}( & \left.C_{\text {sd }}<R \mid H_{0}, \hat{H}=H_{0}\right) \operatorname{Pr}\left(H_{0} \mid \hat{H}=H_{0}\right) \\
& +\operatorname{Pr}\left(C_{s d}<R \mid H_{1}, \hat{H}=H_{0}\right) \operatorname{Pr}\left(H_{1} \mid \hat{H}=H_{0}\right) .
\end{aligned}
$$

453 It is shown from (2) that given $H_{0}$ and $H_{1}$, the parameter $\alpha$ is 454 obtained as $\alpha=0$ and $\alpha=1$, respectively. Thus, combining (2) and (4), we have $C_{s d}=\log _{2}\left(1+\left|h_{s d}\right|^{2} \gamma_{s}\right)$ given $H_{0}$ and $C_{s d}=455$ $\log _{2}\left(1+\frac{\left|h_{s d}\right|^{2} \gamma_{s}}{\left|h_{p d}\right|^{2} \gamma_{p}+1}\right)$ given $H_{1}$. Substituting this result into (29) 456 yields

$$
\begin{aligned}
P_{\text {out }}^{\text {direct }}= & \operatorname{Pr}\left(\left|h_{s d}\right|^{2} \gamma_{s}<2^{R}-1\right) \operatorname{Pr}\left(H_{0} \mid \hat{H}=H_{0}\right) \\
& +\operatorname{Pr}\left(\frac{\left|h_{s d}\right|^{2} \gamma_{s}}{\left|h_{p d}\right|^{2} \gamma_{p}+1}<2^{R}-1\right) \operatorname{Pr}\left(H_{1} \mid \hat{H}=H_{0}\right)
\end{aligned}
$$

Moreover, the terms $\operatorname{Pr}\left(H_{0} \mid \hat{H}=H_{0}\right)$ and $\operatorname{Pr}\left(H_{1} \mid \hat{H}=H_{0}\right)$ can be 458 obtained by using Bayes' theorem as

$$
\begin{aligned}
\operatorname{Pr}\left(H_{0} \mid \hat{H}=H_{0}\right) & =\frac{\operatorname{Pr}\left(\hat{H}=H_{0} \mid H_{0}\right) \operatorname{Pr}\left(H_{0}\right)}{\sum_{i \in\{0,1\}} \operatorname{Pr}\left(\hat{H}=H_{0} \mid H_{i}\right) \operatorname{Pr}\left(H_{i}\right)} \\
& =\frac{P_{0}\left(1-P_{f}\right)}{P_{0}\left(1-P_{f}\right)+\left(1-P_{0}\right)\left(1-P_{d}\right)} \triangleq \pi_{0},
\end{aligned}
$$

and

$$
\operatorname{Pr}\left(H_{1} \mid \hat{H}=H_{0}\right)=\frac{\left(1-P_{0}\right)\left(1-P_{d}\right)}{P_{0}\left(1-P_{f}\right)+\left(1-P_{0}\right)\left(1-P_{d}\right)} \triangleq \pi_{1},
$$

where $P_{0}=\operatorname{Pr}\left(H_{0}\right)$ is the probability that the licensed spec- 461 trum band is unoccupied by $\mathrm{PBS}$, while $P_{d}=\operatorname{Pr}\left(\hat{H}=H_{1} \mid H_{1}\right) 462$ and $P_{f}=\operatorname{Pr}\left(\hat{H}=H_{1} \mid H_{0}\right)$ are the SDP and FAP, respectively. 463 For notational convenience, we introduce the shorthand $\pi_{0}=464$ $\operatorname{Pr}\left(H_{0} \mid \hat{H}=H_{0}\right), \pi_{1}=\operatorname{Pr}\left(H_{1} \mid \hat{H}=H_{0}\right)$ and $\Delta=\frac{2^{R}-1}{\gamma_{s}}$. Then, 465 using (31) and (32), we rewrite (30) as 466

$P_{\text {out }}^{\text {direct }}=\pi_{0} \operatorname{Pr}\left(\left|h_{s d}\right|^{2}<\Delta\right)+\pi_{1} \operatorname{Pr}\left(\left|h_{s d}\right|^{2}-\left|h_{p d}\right|^{2} \gamma_{p} \Delta<\Delta\right)$.

Noting that $\left|h_{s d}\right|^{2}$ and $\left|h_{p d}\right|^{2}$ are independently and exponen- 467 tially distributed RVs with respective means of $\sigma_{s d}^{2}$ and $\sigma_{p d}^{2}, 468$ we obtain

$$
\operatorname{Pr}\left(\left|h_{s d}\right|^{2}<\Delta\right)=1-\exp \left(-\frac{\Delta}{\sigma_{s d}^{2}}\right)
$$

and

$$
\operatorname{Pr}\left(\left|h_{s d}\right|^{2}-\left|h_{p d}\right|^{2} \gamma_{p} \Delta<\Delta\right)=1-\frac{\sigma_{s d}^{2}}{\sigma_{p d}^{2} \gamma_{p} \Delta+\sigma_{s d}^{2}} \exp \left(-\frac{\Delta}{\sigma_{s d}^{2}}\right) .
$$

Additionally, we observe from (26) that an intercept event 471 occurs, when the capacity of the ST-E channel becomes higher 472 than the data rate. Thus, given that a spectrum hole has been de- 473 tected (i.e. $\hat{H}=H_{0}$ ), ST starts transmitting its signal to SD and 474 E may overhear the ST-SD transmission. The corresponding IP 475 is given by

$$
P_{\text {int }}^{\text {direct }}=\operatorname{Pr}\left(C_{s e}>R \mid \hat{H}=H_{0}\right),
$$

which can be further expressed as

$$
\begin{aligned}
P_{\text {int }}^{\text {direct }}= & \operatorname{Pr}\left(C_{s e}>R \mid \hat{H}=H_{0}, H_{0}\right) \operatorname{Pr}\left(H_{0} \mid \hat{H}=H_{0}\right) \\
& +\operatorname{Pr}\left(C_{s e}>R \mid \hat{H}=H_{0}, H_{1}\right) \operatorname{Pr}\left(H_{1} \mid \hat{H}=H_{0}\right) \\
= & \pi_{0} \operatorname{Pr}\left(\left|h_{s e}\right|^{2}>\Delta\right)+\pi_{1} \operatorname{Pr}\left(\left|h_{s e}\right|^{2}-\left|h_{p e}\right|^{2} \gamma_{p} \Delta>\Delta\right),
\end{aligned}
$$


478 where the second equality is obtained by using $C_{s e}$ from (5). 479 Noting that RVs $\left|h_{s e}\right|^{2}$ and $\left|h_{p e}\right|^{2}$ are exponentially distributed 480 and independent of each other, we can express the terms $481 \operatorname{Pr}\left(\left|h_{s e}\right|^{2}>\Delta\right)$ and $\operatorname{Pr}\left(\left|h_{s e}\right|^{2}-\left|h_{p e}\right|^{2} \gamma_{p} \Delta>\Delta\right)$ as

$$
\operatorname{Pr}\left(\left|h_{s e}\right|^{2}>\Delta\right)=\exp \left(-\frac{\Delta}{\sigma_{s e}^{2}}\right)
$$

482 and

$$
\operatorname{Pr}\left(\left|h_{s e}\right|^{2}-\left|h_{p e}\right|^{2} \gamma_{p} \Delta>\Delta\right)=\frac{\sigma_{s e}^{2}}{\sigma_{p e}^{2} \gamma_{p} \Delta+\sigma_{s e}^{2}} \exp \left(-\frac{\Delta}{\sigma_{s e}^{2}}\right)
$$

483 where $\sigma_{s e}^{2}$ and $\sigma_{p e}^{2}$ are the expected values of $\mathrm{RVs}\left|h_{s e}\right|^{2}$ and $484\left|h_{p e}\right|^{2}$, respectively.

\section{B. Single-Relay Selection}

486 In this subsection, we present the SRT analysis of the pro487 posed SRS scheme. Given $\hat{H}=H_{0}$, the OP of the cognitive 488 transmission relying on SRS is given by

$$
\begin{aligned}
P_{\text {out }}^{\text {single }}=\operatorname{Pr}\left(C_{b d}<\right. & \left.R, \mathcal{D}=\emptyset \mid \hat{H}=H_{0}\right) \\
& +\sum_{n=1}^{2^{N}-1} \operatorname{Pr}\left(C_{b d}<R, \mathcal{D}=\mathcal{D}_{n} \mid \hat{H}=H_{0}\right),
\end{aligned}
$$

489 where $C_{b d}$ represents the capacity of the channel from the 490 "best" SR to SD. In the case of $\mathcal{D}=\emptyset$, no $\mathrm{SR}$ is chosen to 491 forward the source signal, which leads to $C_{b d}=0$ for $\mathcal{D}=\emptyset$. 492 Substituting this result into (40) gives

$$
\begin{aligned}
P_{\text {out }}^{\text {single }}=\operatorname{Pr}(\mathcal{D}=\emptyset \mid & \left.\mid \hat{H}=H_{0}\right) \\
& +\sum_{n=1}^{2^{N}-1} \operatorname{Pr}\left(C_{b d}<R, \mathcal{D}=\mathcal{D}_{n} \mid \hat{H}=H_{0}\right) .
\end{aligned}
$$

493 Using (2), (9), (10), and (14), we can rewrite (41) as (42), 494 shown at the bottom of the page, where $\Lambda=\frac{2^{2 R}-1}{\gamma_{s}}$. Noting 495 that $\left|h_{s i}\right|^{2}$ and $\left|h_{p i}\right|^{2}$ are independent exponentially distributed random variables with respective means of $\sigma_{s i}^{2}$ and $\sigma_{p i}^{2}$, we have 496

$$
\operatorname{Pr}\left(\left|h_{s i}\right|^{2}<\Lambda\right)=1-\exp \left(-\frac{\Lambda}{\sigma_{s i}^{2}}\right)
$$

and

$\operatorname{Pr}\left(\left|h_{s i}\right|^{2}<\Lambda\left|h_{p i}\right|^{2} \gamma_{p}+\Lambda\right)=1-\frac{\sigma_{s i}^{2}}{\sigma_{p i}^{2} \gamma_{p} \Lambda+\sigma_{s i}^{2}} \exp \left(-\frac{\Lambda}{\sigma_{s i}^{2}}\right)$,

where the terms $\operatorname{Pr}\left(\left|h_{s i}\right|^{2}>\Lambda\right), \operatorname{Pr}\left(\left|h_{s j}\right|^{2}<\Lambda\right)$, and $\operatorname{Pr}\left(\left|h_{s i}\right|^{2}>498\right.$ $\left.\Lambda\left|h_{p i}\right|^{2} \gamma_{p}+\Lambda\right)$ can be similarly determined in closed-form. 499 Moreover, based on Appendix A, we obtain $\operatorname{Pr}\left(\max _{i \in \mathcal{D}_{n}}\left|h_{i d}\right|^{2}<\Lambda\right) 500$ and $\operatorname{Pr}\left(\max _{i \in \mathcal{D}_{n}}\left|h_{i d}\right|^{2}<\Lambda\left|h_{p d}\right|^{2} \gamma_{p}+\Lambda\right)$ as

$$
\operatorname{Pr}\left(\max _{i \in \mathcal{D}_{n}}\left|h_{i d}\right|^{2}<\Lambda\right)=\prod_{i \in \mathcal{D}_{n}}\left[1-\exp \left(-\frac{\Lambda}{\sigma_{i d}^{2}}\right)\right],
$$

and

$$
\begin{aligned}
\operatorname{Pr}\left(\max _{i \in \mathcal{D}_{n}}\left|h_{i d}\right|^{2}<\Lambda\left|h_{p d}\right|^{2} \gamma_{p}+\Lambda\right) \\
=1+\sum_{m=1}^{2\left|\mathcal{D}_{n}\right|}(-1)^{\left|\tilde{\mathcal{D}}_{n}(m)\right|} \exp \left(-\sum_{i \in \tilde{\mathcal{D}}_{n}(m)} \frac{\Lambda}{\sigma_{i d}^{2}}\right) \\
\times\left(1+\sum_{i \in \tilde{\mathcal{D}}_{n}(m)} \frac{\Lambda \gamma_{p} \sigma_{p d}^{2}}{\sigma_{i d}^{2}}\right)^{-1},
\end{aligned}
$$

where $\tilde{D}_{n}(m)$ represents the $m$-th non-empty subset of $\mathcal{D}_{n} .503$ Additionally, the IP of the SRS scheme can be expressed as 504

$$
\begin{aligned}
P_{\text {int }}^{\text {single }}=\operatorname{Pr}\left(C_{b e}>\right. & \left.R, \mathcal{D}=\emptyset \mid \hat{H}=H_{0}\right) \\
& +\sum_{n=1}^{2^{N}-1} \operatorname{Pr}\left(C_{b e}>R, \mathcal{D}=\mathcal{D}_{n} \mid \hat{H}=H_{0}\right),
\end{aligned}
$$

where $C_{b e}$ represents the capacity of the channel spanning from 505 the "best" SR to E. Given $\mathcal{D}=\emptyset$, we have $C_{b e}=0$, since 506 no relay is chosen for forwarding the source signal. Thus, 507

$$
\begin{aligned}
P_{\text {out }}^{\text {single }}= & \pi_{0} \prod_{i=1}^{N} \operatorname{Pr}\left(\left|h_{s i}\right|^{2}<\Lambda\right)+\pi_{1} \prod_{i=1}^{N} \operatorname{Pr}\left(\left|h_{s i}\right|^{2}<\Lambda\left|h_{p i}\right|^{2} \gamma_{p}+\Lambda\right) \\
& +\pi_{0} \sum_{n=1}^{2^{N}-1} \prod_{i \in \mathcal{D}_{n}} \operatorname{Pr}\left(\left|h_{s i}\right|^{2}>\Lambda\right) \prod_{j \in \overline{\mathcal{D}}_{n}} \operatorname{Pr}\left(\left|h_{s j}\right|^{2}<\Lambda\right) \operatorname{Pr}\left(\max _{i \in \mathcal{D}_{n}}\left|h_{i d}\right|^{2}<\Lambda\right) \\
+ & \pi_{1} \sum_{n=1}^{2^{N}-1} \prod_{i \in \mathcal{D}_{n}} \operatorname{Pr}\left(\left|h_{s i}\right|^{2}>\Lambda\left|h_{p i}\right|^{2} \gamma_{p}+\Lambda\right) \prod_{j \in \overline{\mathcal{D}}_{n}} \operatorname{Pr}\left(\left|h_{s j}\right|^{2}<\Lambda\left|h_{p j}\right|^{2} \gamma_{p}+\Lambda\right) \\
& \times \operatorname{Pr}\left(\max _{i \in \mathcal{D}_{n}}\left|h_{i d}\right|^{2}<\Lambda\left|h_{p d}\right|^{2} \gamma_{p}+\Lambda\right)
\end{aligned}
$$


508 substituting this result into (47) and using (2), (9), (10), and 509 (16), we arrive at

$$
\begin{aligned}
& P_{\text {int }}^{\text {single }}=\pi_{0} \sum_{n=1}^{2^{N}-1} \prod_{i \in \mathcal{D}_{n}} \operatorname{Pr}\left(\left|h_{s i}\right|^{2}>\Lambda\right) \prod_{j \in \overline{\mathcal{D}}_{n}} \operatorname{Pr}\left(\left|h_{s j}\right|^{2}<\Lambda\right) \\
& \times \operatorname{Pr}\left(\left|h_{b e}\right|^{2}>\Lambda\right) \\
&+\pi_{1} \sum_{n=1}^{2^{N}-1} \prod_{i \in \mathcal{D}_{n}} \operatorname{Pr}\left(\left|h_{s i}\right|^{2}>\Lambda\left|h_{p i}\right|^{2} \gamma_{p}+\Lambda\right) \\
& \times \prod_{j \in \overline{\mathcal{D}}_{n}} \operatorname{Pr}\left(\left|h_{s j}\right|^{2}<\Lambda\left|h_{p j}\right|^{2} \gamma_{p}+\Lambda\right) \\
& \times \operatorname{Pr}\left(\left|h_{b e}\right|^{2}>\Lambda\left|h_{p e}\right|^{2} \gamma_{p}+\Lambda\right)
\end{aligned}
$$

510 where the closed-form expressions of $\operatorname{Pr}\left(\left|h_{s i}\right|^{2}>\Lambda\right)$ and $511 \operatorname{Pr}\left(\left|h_{s i}\right|^{2}>\Lambda\left|h_{p i}\right|^{2} \gamma_{p}+\Lambda\right)$ can be readily obtained by using 512 (43) and (44). Using the results in Appendix B, we can express $513 \operatorname{Pr}\left(\left|h_{b e}\right|^{2}>\Lambda\right)$ and $\operatorname{Pr}\left(\left|h_{b e}\right|^{2}>\Lambda\left|h_{p e}\right|^{2} \gamma_{p}+\Lambda\right)$ as

$$
\begin{aligned}
& \operatorname{Pr}\left(\left|h_{b e}\right|^{2}>\Lambda\right)=\sum_{i \in \mathcal{D}_{n}} \exp \left(-\frac{\Lambda}{\sigma_{i e}^{2}}\right) \\
& \times\left[1+\sum_{m=1}^{{ }^{\left|\mathcal{D}_{n}\right|-1}-1}(-1)^{\left|C_{n}(m)\right|}\left(1+\sum_{j \in C_{n}(m)} \frac{\sigma_{i d}^{2}}{\sigma_{j d}^{2}}\right)^{-1}\right],
\end{aligned}
$$

514 and

$$
\begin{gathered}
\operatorname{Pr}\left(\left|h_{b e}\right|^{2}>\Lambda\left|h_{p e}\right|^{2} \gamma_{p}+\Lambda\right)=\sum_{i \in \mathcal{D}_{n}} \frac{\sigma_{i e}^{2}}{\sigma_{p e}^{2} \gamma_{p} \Lambda+\sigma_{i e}^{2}} \exp \left(-\frac{\Lambda}{\sigma_{i e}^{2}}\right) \\
\times\left[1+\sum_{m=1}^{{ }^{\left|\mathcal{D}_{n}\right|-1}-1}(-1)^{\left|C_{n}(m)\right|}\left(1+\sum_{j \in C_{n}(m)} \frac{\sigma_{i d}^{2}}{\sigma_{j d}^{2}}\right)^{-1}\right],
\end{gathered}
$$

515 where $C_{n}(m)$ represents the $m$-th non-empty subset of $\mathcal{D}_{n}-\{i\}$ 516 and ' - ' represents the set difference.

\section{C. Multi-Relay Selection}

518 This subsection analyzes the SRT of our MRS scheme for 519 transmission over Rayleigh fading channels. Similarly to (41), the $\mathrm{OP}$ in this case is given by

$$
\begin{aligned}
P_{\text {out }}^{\text {mult }}=\operatorname{Pr}(\mathcal{D} & \left.=\emptyset \mid \hat{H}=H_{0}\right) \\
& +\sum_{n=1}^{2^{N}-1} \operatorname{Pr}\left(C_{d}^{\text {multi }}<R, \mathcal{D}=\mathcal{D}_{n} \mid \hat{H}=H_{0}\right) .
\end{aligned}
$$

Using (2), (9), (10) and (23), we can rewrite (51) as (52), shown 521 at the bottom of the page, where the closed-form expressions 522 of $\operatorname{Pr}\left(\left|h_{s i}\right|^{2}<\Lambda\right), \operatorname{Pr}\left(\left|h_{s i}\right|^{2}<\Lambda\left|h_{p i}\right|^{2} \gamma_{p}+\Lambda\right), \operatorname{Pr}\left(\left|h_{s i}\right|^{2}>\Lambda\right), 523$ $\operatorname{Pr}\left(\left|h_{s j}\right|^{2}<\Lambda\right)$ and $\operatorname{Pr}\left(\left|h_{s i}\right|^{2}>\Lambda\left|h_{p i}\right|^{2} \gamma_{p}+\Lambda\right)$ can be readily 524 derived, as shown in (43) and (44). However, it is challenging 525 to obtain the closed-form expressions of $\operatorname{Pr}\left(\sum_{i \in \mathcal{D}_{1}}\left|h_{i d}\right|^{2}<\Lambda\right)$ and 526 $\operatorname{Pr}\left(\sum_{i \in \mathcal{D}_{n}}\left|h_{i d}\right|^{2}<\gamma_{p} \Lambda\left|h_{p d}\right|^{2}+\Lambda\right)$. For simplicity, we assume that 527 the fading coefficients of all SRs-SD channels, i.e. $\left|h_{i d}\right|^{2}$ for 528 $i \in\{1,2, \cdots, N\}$, are i.i.d. RVs having the same mean (average 529 channel gain) denoted by $\sigma_{d}^{2}=E\left(\left|h_{i d}\right|^{2}\right)$. This assumption is 530 widely used in the cooperative relaying literature and it is 531 valid in a statistical sense, provided that all SRs are uniformly 532 distributed over a certain geographical area. Assuming that 533 RVs of $\left|h_{i d}\right|^{2}$ for $i \in \mathcal{D}_{n}$ are i.i.d., based on Appendix C, 534 we arrive at

$$
\operatorname{Pr}\left(\sum_{i \in \mathcal{D}_{n}}\left|h_{i d}\right|^{2}<\Lambda\right)=\Gamma\left(\frac{\Lambda}{\sigma_{d}^{2}},\left|\mathcal{D}_{n}\right|\right),
$$

$$
\begin{aligned}
& \text { and } \\
& \begin{array}{r}
\operatorname{Pr}\left(\sum_{i \in \mathcal{D}_{n}}\left|h_{i d}\right|^{2}<\gamma_{p} \Lambda\left|h_{p d}\right|^{2}+\Lambda\right)=\Gamma\left(\frac{\Lambda}{\sigma_{d}^{2}},\left|\mathcal{D}_{n}\right|\right) \\
+\frac{\left[1-\Gamma\left(\Lambda \sigma_{d}^{-2}+\sigma_{p d}^{-2} \gamma_{p}^{-1},\left|\mathcal{D}_{n}\right|\right)\right]}{\left(1+\sigma_{d}^{2} \sigma_{p d}^{-2} \gamma_{p}^{-1} \Lambda^{-1}\right)^{\left|\mathcal{D}_{n}\right|}} e^{1 /\left(\sigma_{p d}^{2} \gamma_{p}\right)}
\end{array}
\end{aligned}
$$

where $\Gamma(x, k)=\int_{0}^{x} \frac{t^{k-1}}{\Gamma(k)} e^{-t} d t$ is known as the incomplete 537 Gamma function [32]. Substituting (53) and (54) into (52) 538 yields a closed-form OP expression for the proposed MRS 539 scheme.

$$
\begin{aligned}
P_{\text {out }}^{\text {multi }}= & \pi_{0} \prod_{i=1}^{N} \operatorname{Pr}\left(\left|h_{s i}\right|^{2}<\Lambda\right)+\pi_{1} \prod_{i=1}^{N} \operatorname{Pr}\left(\left|h_{s i}\right|^{2}<\Lambda\left|h_{p i}\right|^{2} \gamma_{p}+\Lambda\right) \\
+ & \pi_{0} \sum_{n=1}^{2^{N}-1} \prod_{i \in \mathcal{D}_{n}} \operatorname{Pr}\left(\left|h_{s i}\right|^{2}>\Lambda\right) \prod_{j \in \overline{\mathcal{D}}_{n}} \operatorname{Pr}\left(\left|h_{s j}\right|^{2}<\Lambda\right) \operatorname{Pr}\left(\sum_{i \in \mathcal{D}_{n}}\left|h_{i d}\right|^{2}<\Lambda\right) \\
+ & \pi_{1} \sum_{n=1}^{2^{N}-1} \prod_{i \in \mathcal{D}_{n}} \operatorname{Pr}\left(\left|h_{s i}\right|^{2}>\Lambda\left|h_{p i}\right|^{2} \gamma_{p}+\Lambda\right) \prod_{j \in \overline{\mathcal{D}}_{n}} \operatorname{Pr}\left(\left|h_{s j}\right|^{2}<\Lambda\left|h_{p j}\right|^{2} \gamma_{p}+\Lambda\right) \\
& \times \operatorname{Pr}\left(\sum_{i \in \mathcal{D}_{n}}\left|h_{i d}\right|^{2}<\gamma_{p} \Lambda\left|h_{p d}\right|^{2}+\Lambda\right)
\end{aligned}
$$


541 Next, we present the IP analysis of the MRS scheme. Simi542 larly to (48), the IP of the MRS can be obtained from (24) as

$$
\begin{aligned}
P_{\text {int }}^{\text {multi }}=\pi_{0} \sum_{n=1}^{2^{N}-1} \prod_{i \in \mathcal{D}_{n}} \operatorname{Pr}\left(\left|h_{s i}\right|^{2}>\Lambda\right) \prod_{j \in \overline{\mathcal{D}}_{n}} \operatorname{Pr}\left(\left|h_{s j}\right|^{2}<\Lambda\right) \\
\quad \times \operatorname{Pr}\left(\frac{\left|H_{d}^{H} H_{e}\right|^{2}}{\left|H_{d}\right|^{2}}>\Lambda\right) \\
+\pi_{1} \sum_{n=1}^{2^{N}-1} \prod_{i \in \mathcal{D}_{n}} \operatorname{Pr}\left(\left|h_{s i}\right|^{2}>\Lambda\left|h_{p i}\right|^{2} \gamma_{p}+\Lambda\right) \\
\times \prod_{j \in \overline{\mathcal{D}}_{n}} \operatorname{Pr}\left(\left|h_{s j}\right|^{2}<\Lambda\left|h_{p j}\right|^{2} \gamma_{p}+\Lambda\right) \\
\quad \times \operatorname{Pr}\left(\frac{\left|H_{d}^{H} H_{e}\right|^{2}}{\left|H_{d}\right|^{2}}>\gamma_{p} \Lambda\left|h_{p e}\right|^{2}+\Lambda\right),
\end{aligned}
$$

543 where the closed-form expressions of $\operatorname{Pr}\left(\left|h_{s i}\right|^{2}>\Lambda\right)$, $544 \operatorname{Pr}\left(\left|h_{s j}\right|^{2}<\Lambda\right), \operatorname{Pr}\left(\left|h_{s i}\right|^{2}>\Lambda\left|h_{p i}\right|^{2} \gamma_{p}+\Lambda\right)$ and $\operatorname{Pr}\left(\left|h_{s j}\right|^{2}<\right.$ $545 \Lambda\left|h_{p j}\right|^{2} \gamma_{p}+\Lambda$ ) may be readily derived by using (43) and (44). 546 However, it is challenging to obtain the closed-form solutions 547 for $\operatorname{Pr}\left(\frac{\left|H_{d}^{H} H_{e}\right|^{2}}{\left|H_{d}\right|^{2}}>\Lambda\right)$ and $\operatorname{Pr}\left(\frac{\left|H_{d}^{H} H_{e}\right|^{2}}{\left|H_{d}\right|^{2}}>\gamma_{p} \Lambda\left|h_{p e}\right|^{2}+\Lambda\right)$. 548 Although finding a general closed-form IP expression for the 549 MRS scheme is challenging, we can obtain the numerical IP 550 results with the aid of computer simulations.

\section{Numerical RESUlts AND Discussions}

552 In this section, we present our performance comparisons 553 among the direct transmission, the SRS and MRS schemes 554 in terms of their SRT. To be specific, the analytic IP versus 555 OP of the three schemes are obtained by plotting (33), (37), 556 (42), (48), (52), and (55). The simulated IP and OP results of 557 the three schemes are also given to verify the correctness of 558 the theoretical SRT analysis. In our computer simulations, the 559 fading amplitudes (e.g., $\left|h_{s d}\right|,\left|h_{s i}\right|,\left|h_{i d}\right|$, etc.) are first generated 560 based on the Rayleigh distribution having different variances 561 for different channels. Then, the randomly generated fading 562 amplitudes are substituted into the definition of an outage (or 563 intercept) event, which would determine whether an outage (or 564 intercept) event occurs or not. By repeatedly achieving this pro565 cess, we can calculate the relative frequency of occurrence for 566 an outage (intercept) event, which is the simulated OP (or IP). 567 Additionally, the SDP $P_{d}$ and FAP $P_{f}$ are set to $P_{d}=0.99$ 568 and $P_{f}=0.01$, unless otherwise stated. The primary signal569 to-noise ratio (SNR) of $\gamma_{p}=10 \mathrm{~dB}$ and the data rate of $570 R=1 \mathrm{bit} / \mathrm{s} / \mathrm{Hz}$ are used in our numerical evaluations.

571 The artificial noise based method [35], [36] is also consid572 ered for the purpose of numerical comparison with the relay 573 selection schemes. To be specific, in the artificial noise based 574 scheme, ST directly transmits its signal $x_{s}$ to SD, while $N$ SRs 575 attempt to confuse the eavesdropper by sending an interfering 576 signal (referred to as artificial noise) that is approximately 577 designed to lie in the null-space of the legitimate main channel. 578 In this way, the artificial noise will impose interference on the 579 eavesdropper without affecting the SD. For a fair comparison, 580 the total transmit power of the desired signal $x_{s}$ and the artificial 581 noise are constrained to $P_{S}$. Moreover, the equal power alloca582 tion method [35] is used in the numerical evaluation.

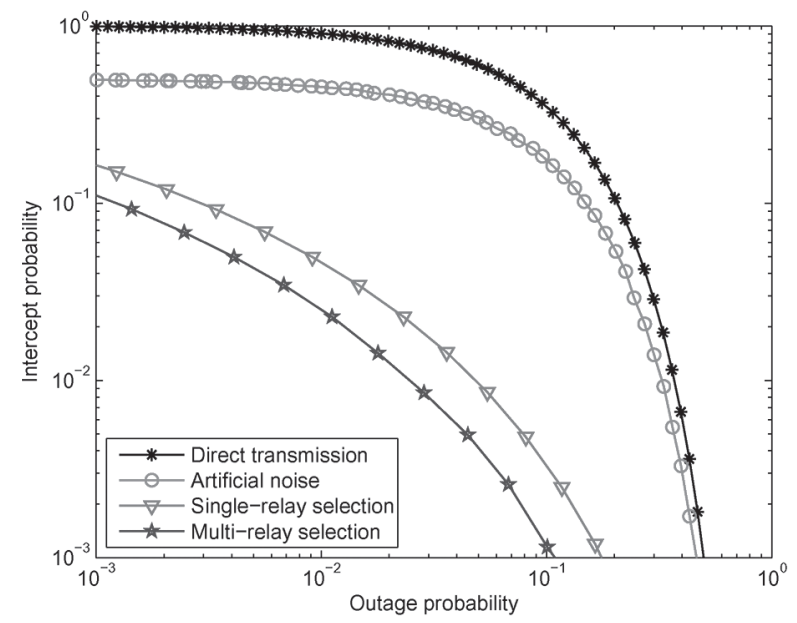

Fig. 3. IP versus OP of the direct transmission, the SRS and the MRS schemes for different $P_{0}$ with $P_{0}=0.8, \gamma_{s} \in[0,35 \mathrm{~dB}], N=6, \sigma_{s d}^{2}=\sigma_{s i}^{2}=\sigma_{i d}^{2}=1$, $\sigma_{s e}^{2}=\sigma_{i e}^{2}=0.1$, and $\sigma_{p d}^{2}=\sigma_{p e}^{2}=\sigma_{p i}^{2}=0.2$.

Fig. 3 shows the IP versus OP of the direct transmission, 583 as well as the SRS and MRS schemes for $P_{0}=0.8$, where 584 the solid lines and discrete marker symbols represent the an- 585 alytic and simulated results, respectively. It can be seen from 586 Fig. 3 that the IP of the direct transmission, the artificial noise 587 based as well as of the proposed SRS and MRS schemes all 588 improve upon tolerating a higher OP, implying that a trade-off 589 exists between the IP (security) and the OP (reliability) of CR 590 transmissions. Fig. 3 also shows that both the proposed SRS 591 and MRS schemes outperform the direct transmission and the 592 artificial noise based approaches in terms of their SRT, showing 593 the advantage of exploiting relay selection against the eaves- 594 dropping attack. Moreover, the SRT performance of the MRS is 595 better than that of the SRS. Although the MRS achieves a better 596 SRT performance than its SRS-aided counterpart, this result 597 is obtained at the cost of a higher implementation complexity, 598 since multiple SRs require high-complexity symbol-level syn- 599 chronization for simultaneously transmitting to the SD, whereas 600 the SRS does not require such elaborate synchronization. 601

Fig. 4 illustrates our numerical SRT comparison between the 602 SRS and MRS schemes for $P_{0}=0.2$ and $P_{0}=0.8$. Observe 603 from Fig. 4 that the MRS scheme performs better than the SRS 604 in terms of its SRT performance for both $P_{0}=0.2$ and $P_{0}=0.8 .605$ It is also seen from Fig. 4 that as $P_{0}$ increases from 0.2 to 606 0.8, the SRT of both the SRS and MRS schemes improves. 607 This is because upon increasing $P_{0}$, the licensed band becomes 608 unoccupied by the PUs with a higher probability and hence the 609 secondary users (SUs) have more opportunities for accessing 610 the licensed band for their data transmissions, which leads 611 to a reduction of the OP for CR transmissions. Meanwhile, 612 increasing $P_{0}$ may simultaneously result in an increase of the IP, 613 since the eavesdropper also has more opportunities for tapping 614 the cognitive transmissions. However, in both the SRS and 615 MRS schemes, the relay selection is performed for the sake 616 of maximizing the legitimate transmission capacity without 617 affecting the eavesdropper's channel capacity. Hence, upon 618 increasing $P_{0}$, it becomes more likely that the reduction of OP 619 is more significant than the increase of IP, hence leading to an 620 overall SRT improvement for the SRS and MRS schemes. 


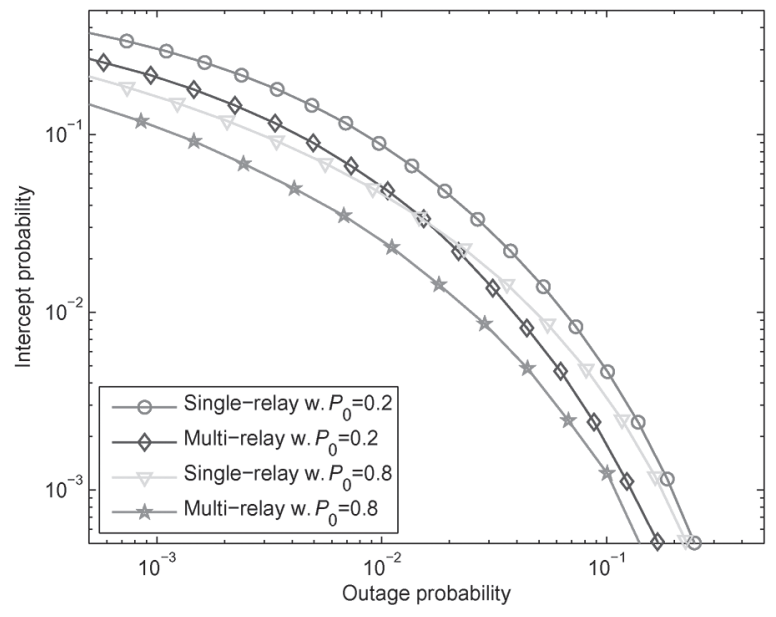

Fig. 4. IP versus OP of the SRS and MRS schemes for different $P_{0}$ with $\gamma_{s} \in[0,30 \mathrm{~dB}], N=6, \sigma_{s d}^{2}=\sigma_{s i}^{2}=\sigma_{i d}^{2}=1, \sigma_{s e}^{2}=\sigma_{i e}^{2}=0.1$, and $\sigma_{p d}^{2}=\sigma_{p e}^{2}=$ $\sigma_{p i}^{2}=0.2$.

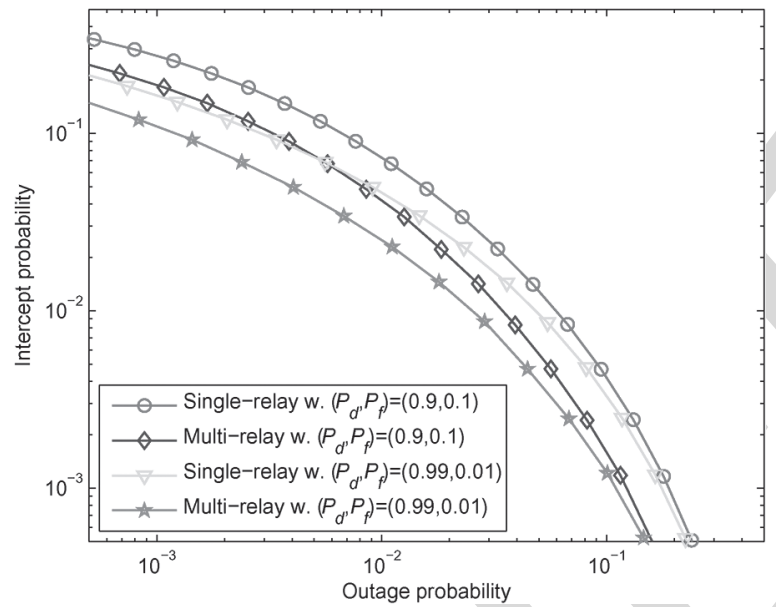

Fig. 5. IP versus OP of the SRS and the MRS schemes for different $\left(P_{d}, P_{f}\right)$ with $P_{0}=0.8, \gamma_{s} \in[0,30 \mathrm{~dB}], N=6, \sigma_{s d}^{2}=\sigma_{s i}^{2}=\sigma_{i d}^{2}=1, \sigma_{s e}^{2}=\sigma_{i e}^{2}=0.1$, and $\sigma_{p d}^{2}=\sigma_{p e}^{2}=\sigma_{p i}^{2}=0.2$.

622 In Fig. 5, we depict the IP versus OP of the SRS and MRS 623 schemes for different spectrum sensing reliabilities, where $624\left(P_{d}, P_{f}\right)=(0.9,0.1)$ and $\left(P_{d}, P_{f}\right)=(0.99,0.01)$ are considered. $625 \mathrm{It}$ is observed that as the spectrum sensing reliability is im626 proved from $\left(P_{d}, P_{f}\right)=(0.9,0.1)$ to $\left(P_{d}, P_{f}\right)=(0.99,0.01)$, the 627 SRTs of the SRS and MRS schemes improve accordingly. This 628 is due to the fact that for an improved sensing reliability, an 629 unoccupied licensed band would be detected more accurately 630 and hence less mutual interference occurs between the PUs 631 and SUs, which results in a better SRT for the secondary 632 transmissions. Fig. 5 also shows that for $\left(P_{d}, P_{f}\right)=(0.9,0.1)$ 633 and $\left(P_{d}, P_{f}\right)=(0.99,0.01)$, the MRS approach outperforms the 634 SRS scheme in terms of the SRT, which further confirms the ad635 vantage of the MRS for protecting the secondary transmissions 636 against eavesdropping attacks.

637 Fig. 6 shows the IP versus OP of the conventional direct 638 transmission as well as of the proposed SRS and MRS schemes 639 for $N=2, N=4$, and $N=8$. It is seen from Fig. 6 that the SRTs

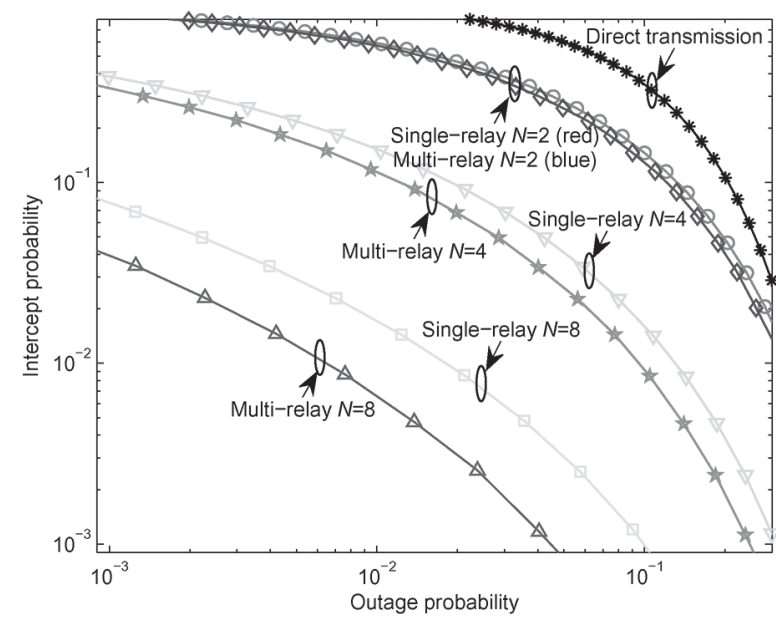

Fig. 6. IP versus $\mathrm{OP}$ of the direct transmission, the $\mathrm{SRS}$ and the MRS schemes for different $N$ with $P_{0}=0.8, \gamma_{s} \in[0,30 \mathrm{~dB}], \sigma_{s d}^{2}=\sigma_{s i}^{2}=\sigma_{i d}^{2}=1, \sigma_{s e}^{2}=\sigma_{i e}^{2}=$ 0.1 , and $\sigma_{p d}^{2}=\sigma_{p e}^{2}=\sigma_{p i}^{2}=0.2$.

of the proposed SRS and MRS schemes are generally better 640 than that of the conventional direct transmission for $N=2,641$ $N=4$ and $N=8$. Moreover, as the number of SRs increases 642 from $N=2$ to 8 , the SRT of the SRS and MRS schemes 643 significantly improves, explicitly demonstrating the security 644 and reliability benefits of exploiting multiple SRs for assisting 645 the secondary transmissions. In other words, the security and 646 reliability of the secondary transmissions can be concurrently 647 improved by increasing the number of SRs. Additionally, as 648 shown in Fig. 6, upon increasing the number of SRs from 649 $N=2$ to 8 , the SRT improvement of MRS over SRS becomes 650 more notable. Again, the SRT advantage of the MRS over the 651 SRS comes at the expense of requiring elaborate symbol-level 652 synchronization among the multiple SRs for simultaneously 653 transmitting to the SD.

\section{CONCLUSION}

In this paper, we proposed relay selection schemes for 656 a CR network consisting of a ST, a SD and multiple SRs 657 communicating in the presence of an eavesdropper. We ex- 658 amined the SRT performance of the SRS and MRS assisted 659 secondary transmissions in the presence of realistic spectrum 660 sensing, where both the security and reliability of secondary 661 transmissions are characterized in terms of their IP and OP, 662 respectively. We also analyzed the SRT of the conventional 663 direct transmission as a benchmark. It was illustrated that as the 664 spectrum sensing reliability increases, the SRTs of both the SRS 665 and MRS schemes improve. We also showed that the proposed 666 SRS and MRS schemes generally outperform the conventional 667 direct transmission and artificial noise based approaches in 668 terms of their SRT. Moreover, the SRT performance of MRS 669 is better than that of SRS. Additionally, as the number of SRs 670 increases, the SRTs of both the SRS and of the MRS schemes 671 improve significantly, demonstrating their benefits in terms 672 of enhancing both the security and reliability of secondary 673 transmissions. 
677 Letting $\left|h_{i d}\right|^{2}=x_{i}$ and $\left|h_{p d}\right|^{2}=y$, the left hand side of (45) 678 and (46) can be rewritten as $\operatorname{Pr}\left(\max _{i \in \mathcal{D}_{n}} x_{i}<\Lambda\right)$ and $\operatorname{Pr}\left(\max _{i \in \mathcal{D}_{n}} x_{i}<\right.$ $\left.679 \Lambda \gamma_{p} y+\Lambda\right)$, respectively. Noting that random variables $\left|h_{i d}\right|^{2}$ and $680\left|h_{p d}\right|^{2}$ are exponentially distributed with respective means $\sigma_{i d}^{2}$ 681 and $\sigma_{p d}^{2}$, and independent of each other, we obtain

$$
\begin{aligned}
\operatorname{Pr}\left(\max _{i \in \mathcal{D}_{n}} x_{i}<\Lambda\right) & =\prod_{i \in \mathcal{D}_{n}} \operatorname{Pr}\left(\left|h_{i d}\right|^{2}<\Lambda\right) \\
& =\prod_{i \in \mathcal{D}_{n}}\left[1-\exp \left(-\frac{\Lambda}{\sigma_{i d}^{2}}\right)\right],
\end{aligned}
$$

682 which is (45). Similarly, the term $\operatorname{Pr}\left(\max _{i \in \mathcal{D}_{n}} x_{i}<\Lambda \gamma_{p} y+\Lambda\right)$ can be 683 computed as

$$
\begin{aligned}
& \operatorname{Pr}\left(\max _{i \in \mathcal{D}_{n}} x_{i}<\Lambda \gamma_{p} y+\Lambda\right) \\
& \quad=\int_{0}^{\infty} \frac{1}{\sigma_{p d}^{2}} \exp \left(-\frac{y}{\sigma_{p d}^{2}}\right) \prod_{i \in \mathcal{D}_{n}}\left(1-\exp \left(-\frac{\Lambda \gamma_{p} y+\Lambda}{\sigma_{i d}^{2}}\right)\right) d y
\end{aligned}
$$

684 wherein $\prod_{i \in \mathcal{D}_{n}}\left(1-\exp \left(-\frac{\Lambda \gamma_{p} y+\Lambda}{\sigma_{i d}^{2}}\right)\right)$ can be further expanded 685 as

$$
\begin{aligned}
\prod_{i \in \mathcal{D}_{n}}\left(1-\exp \left(-\frac{\Lambda \gamma_{p} y+\Lambda}{\sigma_{i d}^{2}}\right)\right)=1 & \\
& +\sum_{m=1}^{2^{\left|\mathcal{D}_{n}\right|}-1}(-1)^{\left|\tilde{\mathcal{D}}_{n}(m)\right|} \exp \left(-\sum_{i \in \tilde{\mathcal{D}}_{n}(m)} \frac{\Lambda \gamma_{p} y+\Lambda}{\sigma_{i d}^{2}}\right),
\end{aligned}
$$

686 where $\left|\mathcal{D}_{n}\right|$ is the cardinality of set $\mathcal{D}_{n}, \tilde{\mathcal{D}}_{n}(m)$ represents the $687 \mathrm{~m}$-th non-empty subset of $\mathcal{D}_{n}$, and $\left|\tilde{\mathcal{D}}_{n}(m)\right|$ is the cardinality 688 of set $\tilde{\mathcal{D}}_{n}(m)$. Substituting $\prod_{i \in \mathcal{D}_{n}}\left(1-\exp \left(-\frac{\Lambda \gamma_{p} y+\Lambda}{\sigma_{i d}^{2}}\right)\right)$ from 689 (A.3) into (A.2) yields

$$
\begin{aligned}
& \operatorname{Pr}\left(\max _{i \in \mathcal{D}_{n}} x_{i}<\Lambda \gamma_{p} y+\Lambda\right)=\int_{0}^{\infty} \frac{1}{\sigma_{p d}^{2}} \exp \left(-\frac{y}{\sigma_{p d}^{2}}\right) d y \\
& +\sum_{m=1}^{2\left|\mathcal{D}_{n}\right|}(-1)^{\left|\tilde{D}_{n}(m)\right|} \frac{1}{\sigma_{p d}^{2}} \\
& \quad \times \int_{0}^{\infty} \exp \left(-\frac{y}{\sigma_{p d}^{2}}-\sum_{i \in \tilde{\mathcal{D}}_{n}(m)} \frac{\Lambda \gamma_{p} y+\Lambda}{\sigma_{i d}^{2}}\right) d y
\end{aligned}
$$

Finally, performing the integration of (A.4) yields

$$
\begin{aligned}
& \operatorname{Pr}\left(\max _{i \in \mathcal{D}_{n}} x_{i}<\Lambda \gamma_{p} y+\Lambda\right)=1 \\
& \quad+\sum_{m=1}^{2\left|\mathcal{D}_{n}\right|}-1 \\
& \quad \times\left(1+\sum_{i \in \tilde{\mathcal{D}}_{n}(m)} \frac{\Lambda \gamma_{p} \sigma_{p d}^{2}}{\sigma_{i d}^{2}}\right)^{-1} . \tilde{\mathcal{D}}_{n}(m) \mid \exp \left(-\tilde{\mathcal{D}}_{n}(m)\right. \\
& \quad
\end{aligned}
$$

This completes the proof of (45) and (46).

Given $\mathcal{D}=\mathcal{D}_{n}$, any $\mathrm{SR}$ within $\mathcal{D}_{n}$ can be selected as the 694 "best" relay for forwarding the source signal. Thus, using the 695 law of total probability, we have

$$
\begin{aligned}
& \operatorname{Pr}\left(\left|h_{b e}\right|^{2}>\Lambda\right)=\sum_{i \in \mathcal{D}_{n}} \operatorname{Pr}\left(\left|h_{i e}\right|^{2}>\Lambda, b=i\right) \\
& =\sum_{i \in \mathcal{D}_{n}} \operatorname{Pr}\left(\left|h_{i e}\right|^{2}>\Lambda,\left|h_{i d}\right|^{2}>\max _{j \in \mathcal{D}_{n}-\{i\}}\left|h_{j d}\right|^{2}\right) \\
& =\sum_{i \in \mathcal{D}_{n}} \operatorname{Pr}\left(\left|h_{i e}\right|^{2}>\Lambda\right) \operatorname{Pr}\left(\max _{j \in \mathcal{D}_{n}-\{i\}}\left|h_{j d}\right|^{2}<\left|h_{i d}\right|^{2}\right),
\end{aligned}
$$

where in the first line, variable ' $b$ ' stands for the best SR and 697 the second equality is obtained from (13) and '-' represents the 698 set difference. Noting that $\left|h_{i e}\right|^{2}$ is an exponentially distributed 699 random variable with a mean of $\sigma_{i e}^{2}$, we obtain

$$
\operatorname{Pr}\left(\left|h_{i e}\right|^{2}>\Lambda\right)=\exp \left(-\frac{\Lambda}{\sigma_{i e}^{2}}\right) .
$$

Letting $\left|h_{j d}\right|^{2}=x_{j}$ and $\left|h_{i d}\right|^{2}=y$, we have

$$
\begin{aligned}
& \operatorname{Pr}\left(\max _{j \in \mathcal{D}_{n}-\{i\}}\left|h_{j d}\right|^{2}<\left|h_{i d}\right|^{2}\right) \\
& \quad=\int_{0}^{\infty} \frac{1}{\sigma_{i d}^{2}} \exp \left(-\frac{y}{\sigma_{i d}^{2}}\right) \prod_{j \in \mathcal{D}_{n}-\{i\}}\left(1-\exp \left(-\frac{y}{\sigma_{j d}^{2}}\right)\right) d y,
\end{aligned}
$$

$$
\begin{aligned}
& \text { wherein } \prod_{j \in \mathcal{D}_{n}-\{i\}}\left(1-\exp \left(-\frac{y}{\sigma_{j d}^{2}}\right)\right) \text { is expanded by } \\
& \prod_{j \in \mathcal{D}_{n}-\{i\}}\left(1-\exp \left(-\frac{y}{\sigma_{j d}^{2}}\right)\right)=1 \\
& +\sum_{m=1}^{2^{\left|\mathcal{D}_{n}\right|-1}-1}(-1)^{\left|\mathcal{C}_{n}(m)\right|} \exp \left(-\sum_{j \in \mathcal{C}_{n}(m)} \frac{y}{\sigma_{j d}^{2}}\right),
\end{aligned}
$$

where $\left|\mathcal{D}_{n}\right|$ denotes the cardinality of the set $\mathcal{D}_{n}$ and $\mathcal{C}_{n}(m) 703$ represents the $m$-th non-empty subset of " $\mathcal{D}_{n}-\{i\}$ ". Combining 704 (B.3) and (B.4), we obtain

$$
\begin{aligned}
& \operatorname{Pr}\left(\max _{j \in \mathcal{D}_{n}-\{i\}}\left|h_{j d}\right|^{2}<\left|h_{i d}\right|^{2}\right)=1 \\
& \quad+\sum_{m=1}^{2^{\left|\mathcal{D}_{n}\right|-1}-1}(-1)^{\left|\mathcal{C}_{n}(m)\right|}\left(1+\sum_{j \in \mathcal{C}_{n}(m)} \frac{\sigma_{i d}^{2}}{\sigma_{j d}^{2}}\right)^{-1} .
\end{aligned}
$$


706 Substituting (B.2) and (B.5) into (B.1) gives (B.6), shown at 707 the bottom of the page, which is (49). Similarly to (B.1), we 708 can rewrite $\operatorname{Pr}\left(\left|h_{b e}\right|^{2}>\Lambda\left|h_{p e}\right|^{2} \gamma_{p}+\Lambda\right)$ as

$$
\begin{aligned}
& \operatorname{Pr}\left(\left|h_{b e}\right|^{2}>\Lambda\left|h_{p e}\right|^{2} \gamma_{p}+\Lambda\right) \\
& =\sum_{i \in \mathcal{D}_{n}} \operatorname{Pr}\left(\left|h_{i e}\right|^{2}>\Lambda\left|h_{p e}\right|^{2} \gamma_{p}+\Lambda\right) \\
& \quad \times \operatorname{Pr}\left(\max _{j \in\left\{\mathcal{D}_{n}-i\right\}}\left|h_{j d}\right|^{2}<\left|h_{i d}\right|^{2}\right) .
\end{aligned}
$$

709 Since the random variables $\left|h_{i e}\right|^{2}$ and $\left|h_{p e}\right|^{2}$ are independently 710 and exponentially distributed with respective means of $\sigma_{i e}^{2}$ and $711 \sigma_{p e}^{2}$, we readily arrive at

$$
\operatorname{Pr}\left(\left|h_{i e}\right|^{2}>\Lambda\left|h_{p e}\right|^{2} \gamma_{p}+\Lambda\right)=\frac{\sigma_{i e}^{2}}{\sigma_{p e}^{2} \gamma_{p} \Lambda+\sigma_{i e}^{2}} \exp \left(-\frac{\Lambda}{\sigma_{i e}^{2}}\right) .
$$

712 Substituting (B.5) and (B.8) into (B.7) gives (B.9), shown at the 713 bottom of the page, which is (50).

$717\left|h_{p d}\right|^{2}$, we can rewrite the terms $\operatorname{Pr}\left(\sum_{i \in \mathcal{D}_{n}}\left|h_{i d}\right|^{2}<\Lambda\right)$ and $718 \operatorname{Pr}\left(\sum_{i \in \mathcal{D}_{n}}\left|h_{i d}\right|^{2}<\gamma_{p} \Lambda\left|h_{p d}\right|^{2}+\Lambda\right)$ as $\operatorname{Pr}(X<\Lambda)$ and $\operatorname{Pr}(X<$ $719 \gamma_{p} \Lambda Y+\Lambda$ ), respectively. Noting that the fading coefficients of 720 all SR-SD channels, i.e. $\left|h_{i d}\right|^{2}$ for $i \in\{1,2, \cdots, N\}$, are assumed 721 to be i.i.d., we obtain the probability density function (PDF) of $722 X=\sum_{i \in \mathcal{D}_{n}}\left|h_{i d}\right|^{2}$ as

$$
f_{X}(x)=\frac{1}{\Gamma\left(\left|\mathcal{D}_{n}\right|\right) \sigma_{d}^{2\left|\mathcal{D}_{n}\right|}} x^{\left|\mathcal{D}_{n}\right|-1} \exp \left(-\frac{x}{\sigma_{d}^{2}}\right),
$$

723 where $\sigma_{d}^{2}=E\left(\left|h_{i d}\right|^{2}\right)$. Meanwhile, the random variable $Y=$ $724\left|h_{p d}\right|^{2}$ is exponentially distributed and its PDF is given by

$$
f_{Y}(y)=\frac{1}{\sigma_{p d}^{2}} \exp \left(-\frac{y}{\sigma_{p d}^{2}}\right)
$$

where $\sigma_{p d}^{2}=E\left(\left|h_{p d}\right|^{2}\right)$. Using (C.1), we arrive at

$$
\begin{aligned}
\operatorname{Pr}(X<\Lambda) & =\int_{0}^{\Lambda} \frac{1}{\Gamma\left(\left|\mathcal{D}_{n}\right|\right) \sigma_{d}^{2\left|\mathcal{D}_{n}\right|}} x^{\left|\mathcal{D}_{n}\right|-1} \exp \left(-\frac{x}{\sigma_{d}^{2}}\right) d x \\
& =\int_{0}^{\frac{\Lambda}{\sigma_{d}^{2}}} \frac{t^{\left|\mathcal{D}_{n}\right|-1}}{\Gamma\left(\left|\mathcal{D}_{n}\right|\right)} \exp (-t) d t \\
& =\Gamma\left(\frac{\Lambda}{\sigma_{d}^{2}},\left|\mathcal{D}_{n}\right|\right)
\end{aligned}
$$

where the second equality is obtained by substituting $\frac{x}{\sigma_{d}^{2}}=t$ and 726 $\Gamma(a, k)=\int_{0}^{a} \frac{t^{k-1}}{\Gamma(k)} \exp (-t) d t$ is known as the incomplete Gamma 727 function. Additionally, considering that the random variables $X 728$ and $Y$ are independent of each other, we obtain $\operatorname{Pr}\left(X<\gamma_{p} \Lambda Y+729\right.$ $\Lambda)$ as

$\operatorname{Pr}\left(X<\gamma_{p} \Lambda Y+\Lambda\right)=\int_{0}^{\Lambda} f_{X}(x) d x$

$$
+\int_{\Lambda}^{\infty} \int_{\frac{x}{-\gamma_{p} \Lambda}-\frac{1}{\gamma_{p}}}^{\infty} f_{X}(x) f_{Y}(y) d x d y .
$$

Substituting $f_{X}(x)$ and $f_{Y}(y)$ from (C.1) and (C.2) into (C.4) 731 yields

$\operatorname{Pr}\left(X<\gamma_{p} \Lambda Y+\Lambda\right)$

$$
\begin{aligned}
= & \Gamma\left(\frac{\Lambda}{\sigma_{d}^{2}},\left|\mathcal{D}_{n}\right|\right) \\
& +\int_{\Lambda}^{\infty} \frac{e^{1 /\left(\sigma_{p d}^{2} \gamma_{p}\right)} x^{\left|\mathcal{D}_{n}\right|-1}}{\Gamma\left(\left|\mathcal{D}_{n}\right|\right) \sigma_{d}^{2\left|\mathcal{D}_{n}\right|}} \exp \left(-\frac{x}{\sigma_{d}^{2}}-\frac{x}{\sigma_{p d}^{2} \gamma_{p} \Lambda}\right) d x \\
= & \Gamma\left(\frac{\Lambda}{\sigma_{d}^{2}},\left|\mathcal{D}_{n}\right|\right)+\frac{\left[1-\Gamma\left(\Lambda \sigma_{d}^{-2}+\sigma_{p d}^{-2} \gamma_{p}^{-1},\left|\mathcal{D}_{n}\right|\right)\right]}{\left(1+\sigma_{d}^{2} \sigma_{p d}^{-2} \gamma_{p}^{-1} \Lambda^{-1}\right)^{\left|\mathcal{D}_{n}\right|}} e^{1 /\left(\sigma_{p d}^{2} \gamma_{p}\right)},
\end{aligned}
$$

where the second equality is obtained by using $\frac{x}{\sigma_{d}^{2}}+\frac{x}{\sigma_{p d}^{2} \gamma_{p} \Lambda}=t .733$ Hence, we have completed the proof of (53) and (54) as (C.3) 734 and (C.5), respectively.

$$
\operatorname{Pr}\left(\left|h_{b e}\right|^{2}>\Lambda\right)=\sum_{i \in \mathcal{D}_{n}} \exp \left(-\frac{\Lambda}{\sigma_{i e}^{2}}\right)\left[1+\sum_{m=1}^{\left|\mathscr{D}_{n}\right|-1}(-1)^{\left|\mathcal{C}_{n}(m)\right|}\left(1+\sum_{j \in C_{n}(m)} \frac{\sigma_{i d}^{2}}{\sigma_{j d}^{2}}\right)^{-1}\right]
$$

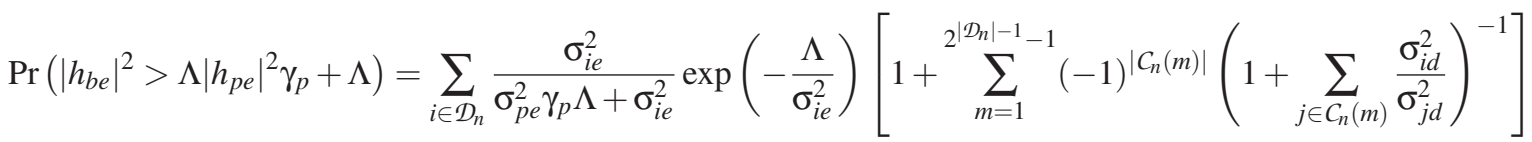




\section{REFERENCES}

1] J. Mitola and G. Q. Maguire, "Cognitive radio: Making software radios more personal," IEEE Pers. Commun., vol. 6, no. 4, pp. 13-18, Aug. 1999. 2] IEEE 802.22 Working Group, IEEE P802.22/D1.0 draft standard for wireless regional area networks part 22: Cognitive wireless RAN medium access control (MAC) and physical layer (PHY) specifications: Policies and procedures for operation in the TV bands, Apr. 2008.

3] G. Baldini, T. Sturman, A. R. Biswas, and R. Leschhorn, "Security aspects in software defined radio and cognitive radio networks: A survey and a way ahead," IEEE Commun. Surveys Tuts., vol. 14, no. 2, pp. 355-379, May 2012.

4] D. Cabric, S. M. Mishra, and R. W. Brodersen, "Implementation issues in spectrum sensing for cognitive radios," in Proc. 38th Asil. Conf. Signal, Syst. Comput., Pacific Grove, CA, USA, Nov. 2004, pp. 772-776.

[5] H. Li, "Cooperative spectrum sensing via belief propagation in spectrumheterogeneous cognitive radio systems," in Proc. IEEE WCNC, Sydney, N.S.W., Australia, Apr. 2010, pp. 1-6.

6] J. Ma, G. Zhao, and Y. Li, "Soft combination and detection for cooperative spectrum sensing in cognitive radio networks," IEEE Trans. Wireless Commun., vol. 7, no. 11, pp. 4502-4507, Nov. 2008.

7] A. Ghasemi and E. S. Sousa, "Fundamental limits of spectrum-sharing in fading environments," IEEE Trans Wireless Commun., vol. 6, no. 2, pp. 649-658, Feb. 2007.

[8] R. Southwell, J. Huang, and X. Liu, "Spectrum mobility games," in Proc. 31st INFOCOM, Orlando, FL, USA, Mar. 2012, pp. 37-45.

9] I. F. Akyildiz, W.-Y. Lee, M. C. Vuran, and S. Mohanty, "A survey on spectrum management in cognitive radio networks," IEEE Commun. Mag., vol. 46, no. 4, pp. 40-48, Apr. 2008.

] H. Li and Z. Han, "Dogfight in spectrum: Combating primary user emulation attacks in cognitive radio systems part I: Known channel statistics," IEEE Trans. Wireless Commun., vol. 9, no. 11, pp. 3566-3577, Nov. 2010.

11] T. Brown and A. Sethi, "Potential cognitive radio denial-of-service vulnerabilities and protection countermeasures: A multi-dimensional analysis and assessment," in Proc. 2nd Int. Conf. CROWNCOM, Orlando, FL, USA, Aug. 2007, pp. 456-464.

2] S. Lakshmanan, C. Tsao, R. Sivakumar, and K. Sundaresan, "Securing wireless data networks against eavesdropping using smart antennas," in Proc. 28th ICDCS, Beijing, China, Jun. 2008, pp. 19-27.

3] A. Olteanu and Y. Xiao, "Security overhead and performance for aggregation with fragment retransmission (AFR) in very high-speed wireless 802.11 LANs," IEEE Trans. Wireless Commun., vol. 9, no. 1, pp. 218226, Jan. 2010.

14] Y. Xiao, V. K. Rayi, X. Du, F. Hu, and M. Galloway, "A survey of key management schemes in wireless sensor networks," Comput. Commun., vol. 30, no. 11-12, pp. 2314-2341, Sep. 2007.

15] A. Mukherjee, S. A. Fakoorian, J. Huang, and A. L. Swindlehurst, "Principles of physical layer security in multiuser wireless networks: A survey," IEEE Commun. Surveys Tuts., vol. 16, no. 3, pp. 1550-1573, Aug. 2014.

6] A. D. Wyner, "The wire-tap channel," Bell Syst. Tech. J., vol. 54, no. 8, pp. 1355-1387, 1975.

17] S. K. Leung-Yan-Cheong and M. E. Hellman, "The Gaussian wiretap channel," IEEE Trans. Inf. Theory, vol. 24, no. 4, pp. 451-456, Jul. 1978.

18] P. Parada and R. Blahut, "Secrecy capacity of SIMO and slow fading channels," in Proc. IEEE ISIT, Adelaide, SA, Australia, Sep. 2005, pp. 2152-2155.

[9] M. Bloch, J. O. Barros, M. R. D. Rodrigues, and S. W. McLaughlin, "Wireless information-theoretic security," IEEE Trans. Inf. Theory, vol. 54, no. 6, pp. 2515-2534, Jun. 2008.

20] P. K. Gopala, L. Lai, and H. Gamal, "On the secrecy capacity of fading channels," IEEE Trans. Inf. Theory, vol. 54, no. 10, pp. 4687-4698, Oct. 2008.

21] Z. Li, W. Trappe, and R. Yates, "Secret communication via multi-antenna transmission," in Proc. 41st Conf. Inf. Sci. Syst., Baltimore, MD, USA, Mar. 2007, pp. 905-910.

22] F. Oggier and B. Hassibi, "The secrecy capacity of the MIMO wiretap channel," IEEE Trans. Inf. Theory, vol. 57, no. 8, pp. 4961-4972, Aug. 2007.

23] M. Yuksel and E. Erkip, "Secure communication with a relay helping the wiretapper," in Proc. IEEE Inf. Theory Workshop, Lake Tahoe, CA, USA, Sep. 2007, pp. 595-600.

4] L. Dong, Z. Han, A. P. Petropulu, and H. V. Poor, "Improving wireless physical layer security via cooperating relays," IEEE Trans. Signal Process., vol. 58, no. 3, pp. 1875-1888, Mar. 2010.
[25] Y. Zou, X. Wang, and W. Shen, "Optimal relay selection for physical-layer 812 security in cooperative wireless networks," IEEE J. Sel. Areas Commun., 813 vol. 31, no. 10, pp. 2099-2111, Oct. 2013.

[26] A. Mukherjee and A. Swindlehurst, "Robust beamforming for security 815 in MIMO wiretap channels with imperfect CSI," IEEE Trans. Signal 816 Process., vol. 59, no. 1, pp. 351-361, Jan. 2011.

[27] C. Jeong, I. Kim, and K. Dong, "Joint secure beamforming design at 818 the source and the relay for an amplify-and-forward MIMO untrusted 819 relay system," IEEE Trans. Signal Process., vol. 60, no. 1, pp. 310-325, 820 Jan. 2012.

[28] Y. Pei, Y.-C. Liang, K. C. Teh, and K. Li, "Secure communication in 822 multiantenna cognitive radio networks with imperfect channel state in- 823 formation," IEEE Trans. Signal Process., vol. 59, no. 4, pp. 1683-1693, 824 Apr. 2011.

[29] Y. Zou, X. Wang, and W. Shen, "Physical-layer security with multiuser 826 scheduling in cognitive radio networks," IEEE Trans. Commun., vol. 61, 827 no. 12 , pp. 5103-5113, Dec. 2013.

[30] Z. Shu, Y. Qian, and S. Ci, "On physical layer security for cognitive radio 829 networks," IEEE Netw. Mag., vol. 27, no. 3, pp. 28-33, Jun. 2013.

[31] Y. Zou, X. Wang, W. Shen, and L. Hanzo, "Security versus reliability 831 analysis of opportunistic relaying," IEEE Trans. Veh. Tech., vol. 63, no. 6, 832 pp. 2653-2661, Jun. 2014.

[32] L. Di Stefano and S. Mattoccia, "A sufficient condition based on the 834 Cauchy-Schwarz inequality for efficient template matching," in Proc. Int. 835 Conf. Image Process., Catalonia, Spain, Sep. 2003, pp. 269-272. 836

[33] M. Abramowitz and I. A. Stegun, Handbook of Mathematical Functions 837 with Formulas, Graphs, Mathematical Tables, 9th ed. New York, NY, 838 USA: Dover, 1970

[34] Y. Zou, Y.-D. Yao, and B. Zheng, "Diversity-multiplexing tradeoff in 840 selective cooperation for cognitive radio," IEEE Trans. Commun., vol. 60, 841 no. 9, pp. 2467-2481, Sep. 2012.

842

[35] S. Goel and R. Negi, "Guaranteeing secrecy using artificial noise," IEEE 843 Trans. Wireless Commun., vol. 7, no. 6, pp. 2180-2189, Jun. 2008.

[36] W. Li, M. Ghogho, B. Chen, and C. Xiong, "Artificial noise by the 845 receiver: Outage secrecy capacity/region analysis," IEEE Commun. Lett., 846 vol. 16, no. 10, pp. 1628-1631, Oct. 2012.

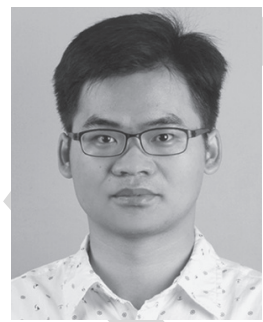

Yulong Zou (S'07-M'12-SM'13) received the 848 B.Eng. degree in information engineering from 849 NUPT, Nanjing, China, in July 2006, the first Ph.D. 850 degree in electrical engineering from the Stevens In- 851 stitute of Technology, New Jersey, the United States, 852 in May 2012, and the second Ph.D. degree in signal 853 and information processing from NUPT, Nanjing, 854 China, in July 2012. He is a Full Professor at the 855 Nanjing University of Posts and Telecommunica- 856 tions (NUPT), Nanjing, China. His research interests 857 span a wide range of topics in wireless commu- 858 nications and signal processing, including the cooperative communications, 859 cognitive radio, wireless security, and energy-efficient communications. $\quad 860$

$\mathrm{He}$ is currently serving as an editor for the IEEE Communications Surveys 861 \& Tutorials, IEEE COMMUNICATIONS LETTERS, EURASIP Journal on Ad- 862 vances in Signal Processing, and KSII Transactions on Internet and Information 863 Systems. He served as the lead guest editor for a special issue on "Security 864 Challenges and Issues in Cognitive Radio Networks" in the EURASIP Journal 865 on Advances in Signal Processing. He is also serving as the lead guest 866 editor for a special issue on "Security and Reliability Challenges in Industrial 867 Wireless Sensor Networks" in the IEEE TRANSACTIONS ON INDUSTRIAL 868 INFORMATICS. In addition, he has acted as symposium chairs, session chairs, 869 and TPC members for a number of IEEE sponsored conferences, including the 870 IEEE WIRELESS COMMUNICATIONS and Networking Conference (WCNC), 871 IEEE Global Communications Conference (GLOBECOM), IEEE International 872 Conference on Communications (ICC), IEEE Vehicular Technology Confer- 873 ence (VTC), International Conference on Communications in China (ICCC), 874 and so on. 


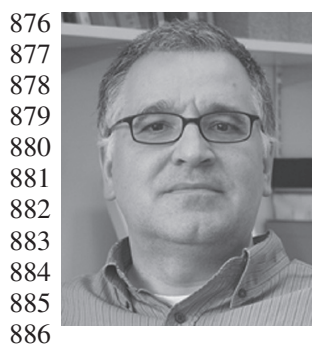

Benoit Champagne (S'87-M'89-SM'03) was born in Joliette (PQ), Canada, in 1961. He received the B.Ing. degree in engineering physics and the M.Sc. degree in physics from the University of Montreal in 1983 and 1985, respectively, and the Ph.D. degree in electrical engineering from the University of Toronto in 1990. From 1990 to 1999 , he was with INRS, University of Quebec, where he held the positions of Assistant and then Associate Professor. In 1999, he joined McGill University, Montreal, as an Associate Professor with the Department of Electrical and 887 Computer Engineering. He served as Associate Chairman of Graduate Studies 888 in the Department from 2004 to 2007 and is now a Full Professor.

889 His research interests focus on the investigation of new computational 890 algorithms for the digital processing of information bearing signals and over891 lap many sub-areas of statistical signal processing, including: detection and 892 estimation, sensor array processing, adaptive filtering, multirate systems, and 893 applications thereof to broadband voice and data communications. Over the 894 years, he has supervised many graduate students in these areas and co-authored 895 several papers, including key works on subspace tracking, speech enhancement, 896 time delay estimation and spread sources localization.

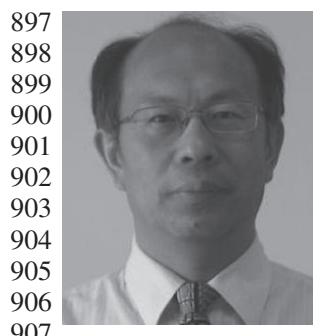

Wei-Ping Zhu (SM'97) received the B.E. and M.E. degrees from Nanjing University of Posts and Telecommunications, and the Ph.D. degree from Southeast University, Nanjing, China, in 1982, 1985, and 1991, respectively, all in electrical engineering. He was a Postdoctoral Fellow from 1991 to 1992 and a Research Associate from 1996 to 1998 with the Department of Electrical and Computer Engineering, Concordia University, Montreal, Canada. During 1993-1996, he was an Associate Professor with the Department of Information Engineering, 908 Nanjing University of Posts and Telecommunications. From 1998 to 2001, he 909 worked with hi-tech companies in Ottawa, Canada, including Nortel Networks 910 and SR Telecom Inc. Since July 2001, he has been with Concordia's Electrical 911 and Computer Engineering Department as a full-time faculty member, where 912 he is presently a Full Professor. His research interests include digital signal 913 processing fundamentals, speech and audio processing, and signal processing 914 for wireless communication with a particular focus on MIMO systems and 915 cooperative relay networks.

916 Dr. Zhu was an Associate Editor of the IEEE TRANSACTIONS ON CIRCUITS 917 AND Systems PART I: Fundamental Theory and Applications from 2001 918 to 2003, and an Associate Editor of Circuits, Systems and Signal Processing 919 from 2006 to 2009. He was also a Guest Editor for the IEEE JOURNAL ON 920 SELECTEd AREAS IN COMMUNiCATIONS for the special issues of: Broadband 921 Wireless Communications for High Speed Vehicles, and Virtual MIMO during 922 2011-2013. Since 2011, he has served as an Associate Editor for the IEEE 923 Transactions on Circuits and Systems Part II: Express Briefs. Dr. 924 Zhu was the Secretary of Digital Signal Processing Technical Committee 925 (DSPTC) of the IEEE Circuits and System Society during 2012-2014, where 926 he is presently the Chair of the DSPTC.

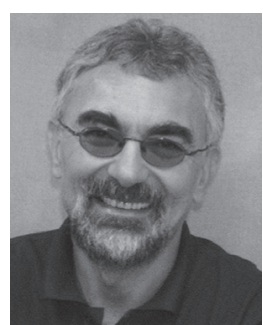

Lajos Hanzo received the degree in electronics in 927 1976 and the doctorate in 1983. In 2009 he was 928 awarded "Doctor Honoris Causa" by the Technical 929 University of Budapest. During his 37-year career in 930 telecommunications he has held various research and 931 academic posts in Hungary, Germany, and the UK. 932 Since 1986 he has been with the School of Electron- 933 ics and Computer Science, University of Southamp- 934 ton, UK, where he holds the chair in telecommunica- 935 tions. He has successfully supervised 80+ Ph.D. stu- 936 dents, co-authored 20 John Wiley/IEEE Press books 937 on mobile radio communications totalling in excess of 10000 pages, published 938 1400+ research entries at IEEE Xplore, acted both as TPC and General Chair of 939 IEEE conferences, presented keynote lectures and has been awarded a number 940 of distinctions. Currently he is directing a 100-strong academic research team, 941 working on a range of research projects in the field of wireless multimedia 942 communications sponsored by industry, the Engineering and Physical Sciences 943 Research Council (EPSRC) UK, the European Research Council's Advanced 944 Fellow Grant and the Royal Society's Wolfson Research Merit Award. He is an 945 enthusiastic supporter of industrial and academic liaison and he offers a range of 946 industrial courses. He is also a Governor of the IEEE VTS. During 2008-2012 947 he was the Editor-in-Chief of the IEEE Press and a Chaired Professor also 948 at Tsinghua University, Beijing. His research is funded by the European 949 Research Council's Senior Research Fellow Grant. For further information on 950 research in progress and associated publications please refer to http://www- 951 mobile.ecs.soton.ac.uk Lajos has $20000+$ citations. 
AUTHOR QUERY

NO QUERY. 